\title{
Iron-catalyzed oxidative decarbonylative $\alpha$-alkylation of acyl- substituted furans with aliphatic aldehydes as the alkylating agents
}

\author{
Wenkun Luo ${ }^{\mathrm{a}}$, Yongjie Yang ${ }^{\mathrm{a}}$, Bo Liu*b and Biaolin Yin*a \\ ${ }^{a}$ Key Laboratory of Functional Molecular Engineering of Guangdong Province, School of \\ Chemistry and Chemical Engineering, South China University of Technology, Guangzhou, P. R. \\ China, 510640,email: blyin@scut.edu.cn \\ ${ }^{b}$ The Second Affiliated Hospital of Guangzhou University of Chinese Medicine, Guangzhou, P.R. \\ China, 510006,e-mail: doctliu@263.net
}




\section{Table of Contents}

I. Copies of ${ }^{1} \mathrm{H}$ and ${ }^{13} \mathrm{C}$ NMR spectra of starting materials and products ............................. 1

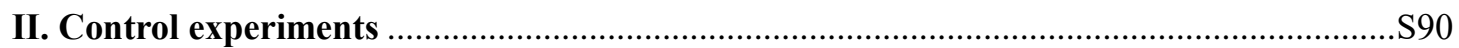


I. Copies of ${ }^{1} \mathrm{H}$ and ${ }^{13} \mathrm{C}$ NMR spectra of starting materials and products

Figure S-1. ${ }^{1} \mathrm{H}$ NMR spectrum of (2a) $\left(\mathrm{CDCl}_{3}, 400 \mathrm{MHz}\right) \boldsymbol{N}$-methyl- $\boldsymbol{N}$-phenylfuran-2-carboxamide

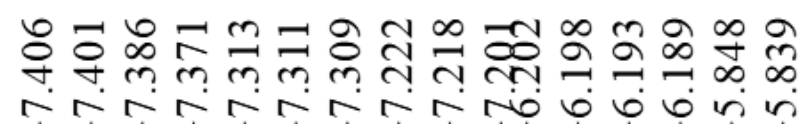

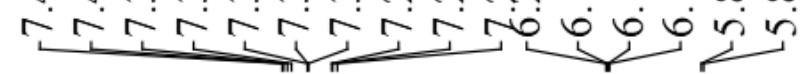
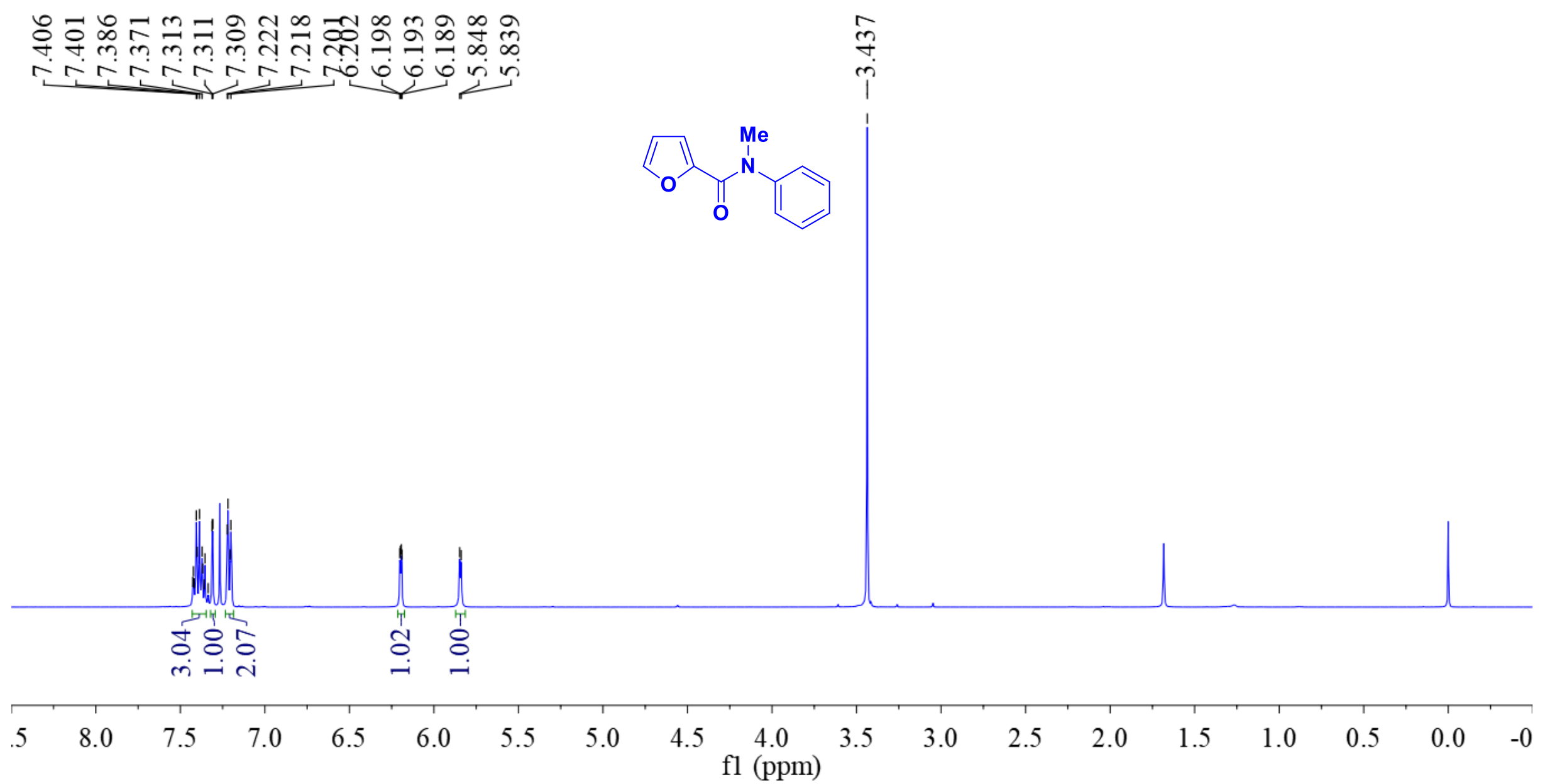
Figure S-2. ${ }^{13} \mathrm{C}\left\{{ }^{1} \mathrm{H}\right\}$ NMR spectrum of (2a) $\left(\mathrm{CDCl}_{3}, 100 \mathrm{MHz}\right) \boldsymbol{N}$-methyl- $N$-phenylfuran-2-carboxamide

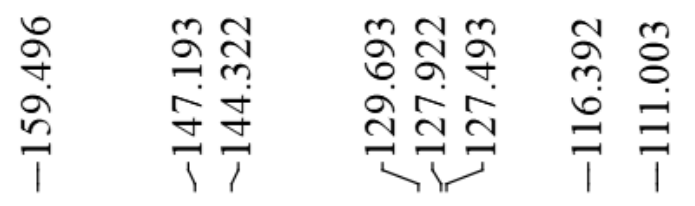
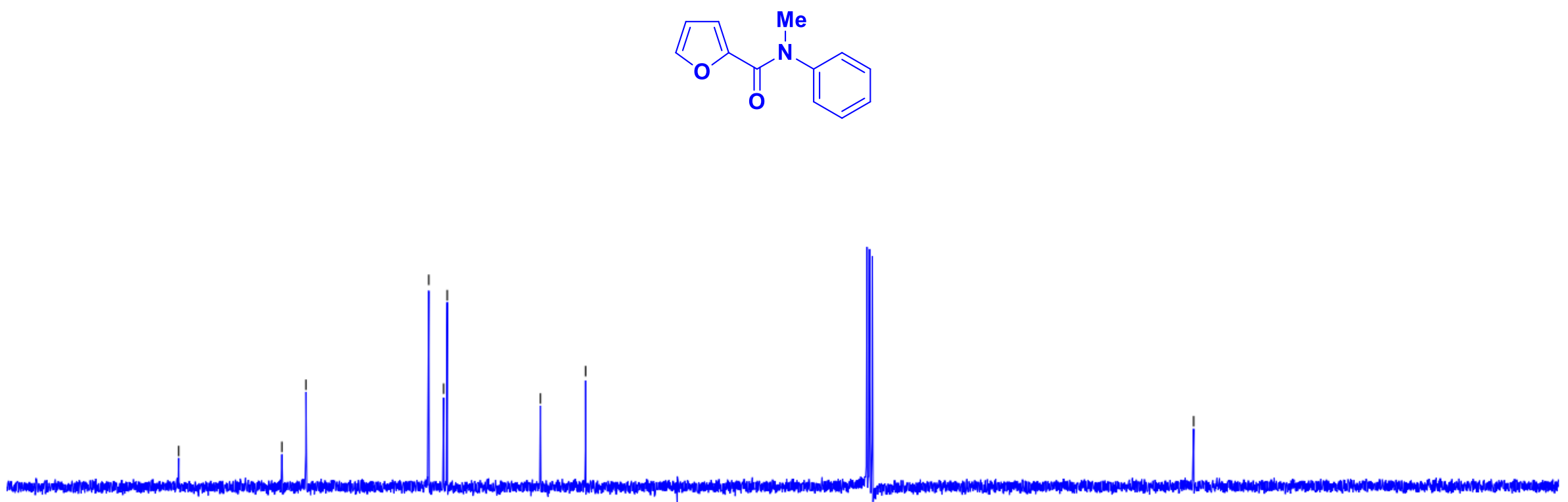

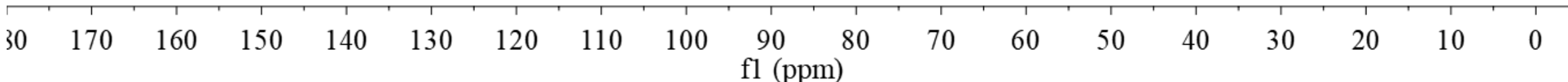


Figure S-3. ${ }^{1} \mathrm{H}$ NMR spectrum of (2b) $\left(\mathrm{CDCl}_{3}, 400 \mathrm{MHz}\right) \mathbf{N}, \boldsymbol{N}$-dimethylfuran-2-carboxamide

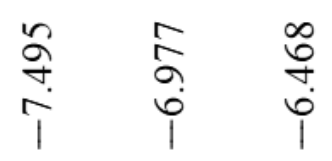

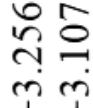

ip
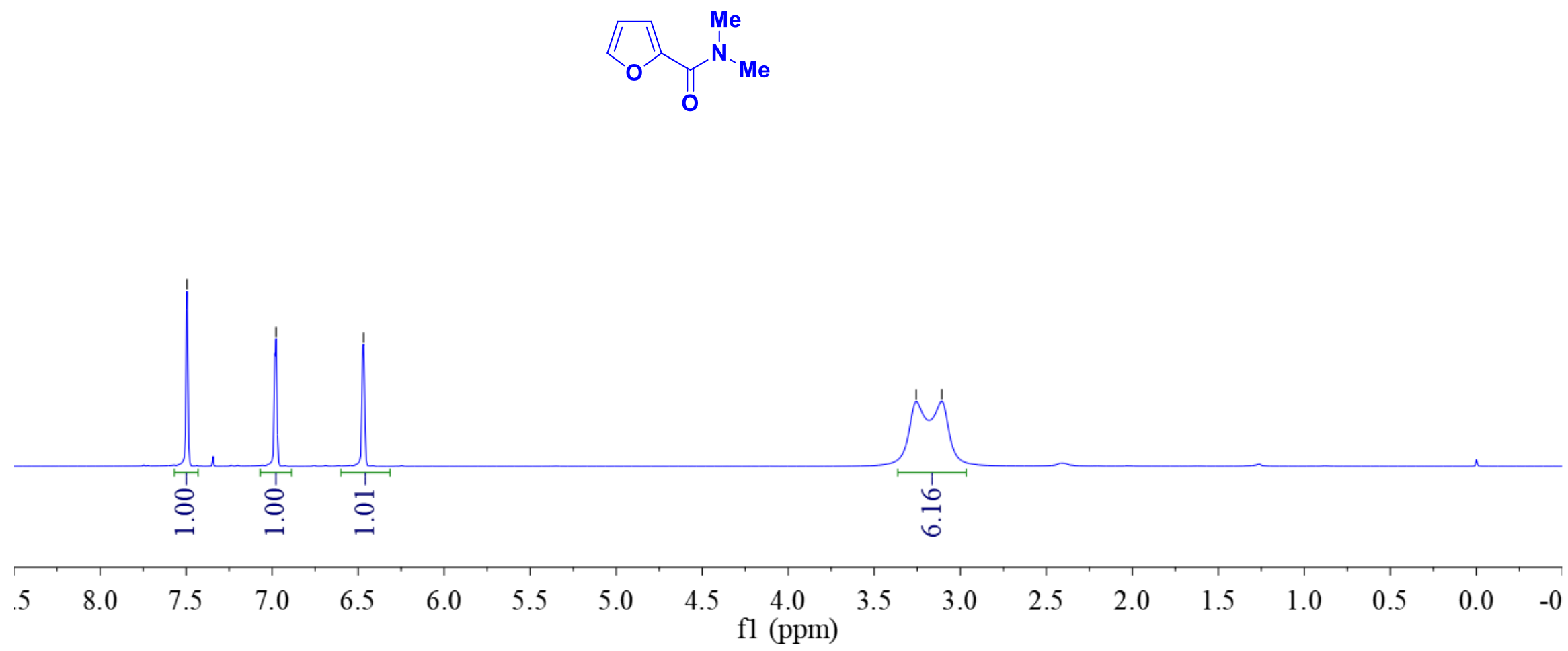
Figure S-4. ${ }^{13} \mathrm{C}\left\{{ }^{1} \mathrm{H}\right\}$ NMR spectrum of $(\mathbf{2 b})\left(\mathrm{CDCl}_{3}, 100 \mathrm{MHz}\right) \mathbf{N}, \mathbf{N}$-dimethylfuran-2-carboxamide

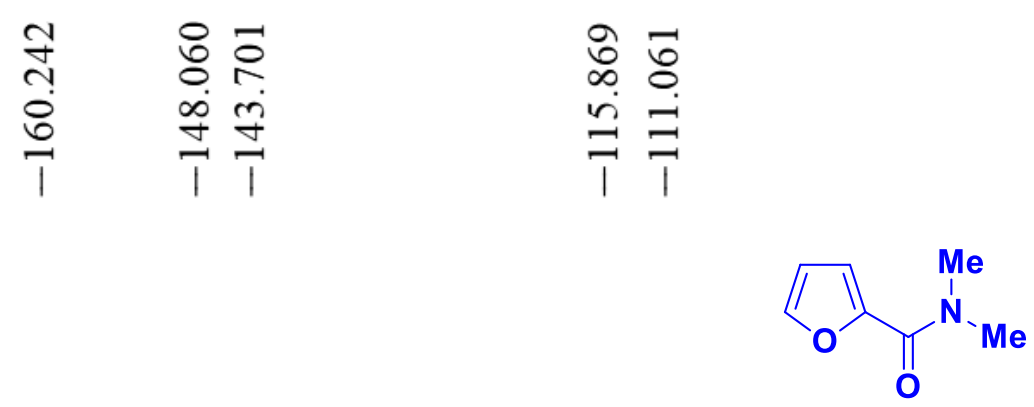

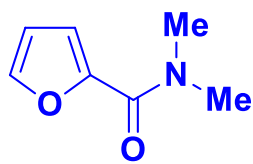

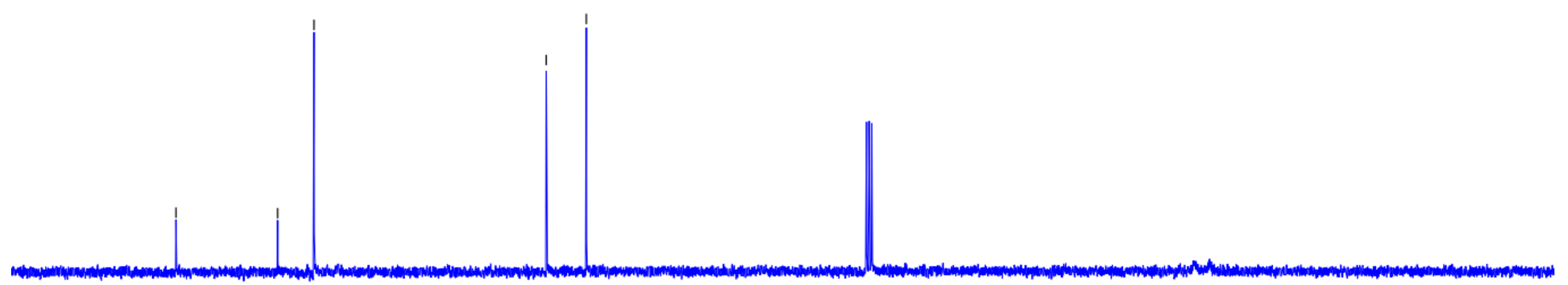

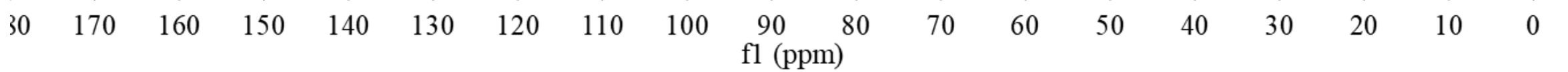


Figure S-5. ${ }^{1} \mathrm{H}$ NMR spectrum of (2c) $\left(\mathrm{CDCl}_{3}, 400 \mathrm{MHz}\right) \mathbf{N}, \mathbf{N}$-diethylfuran-2-carboxamide

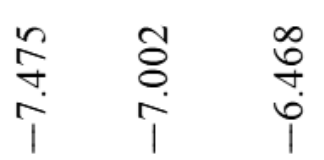
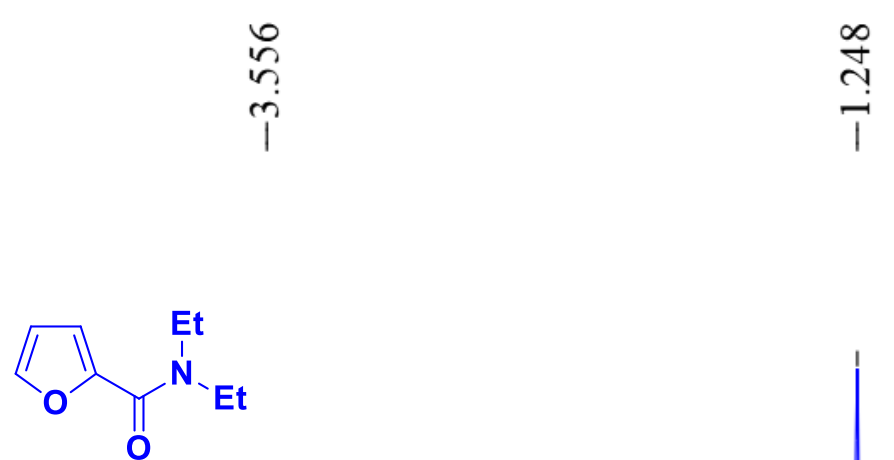

$\stackrel{\substack{i \\ ָ}}{\stackrel{\infty}{i}}$

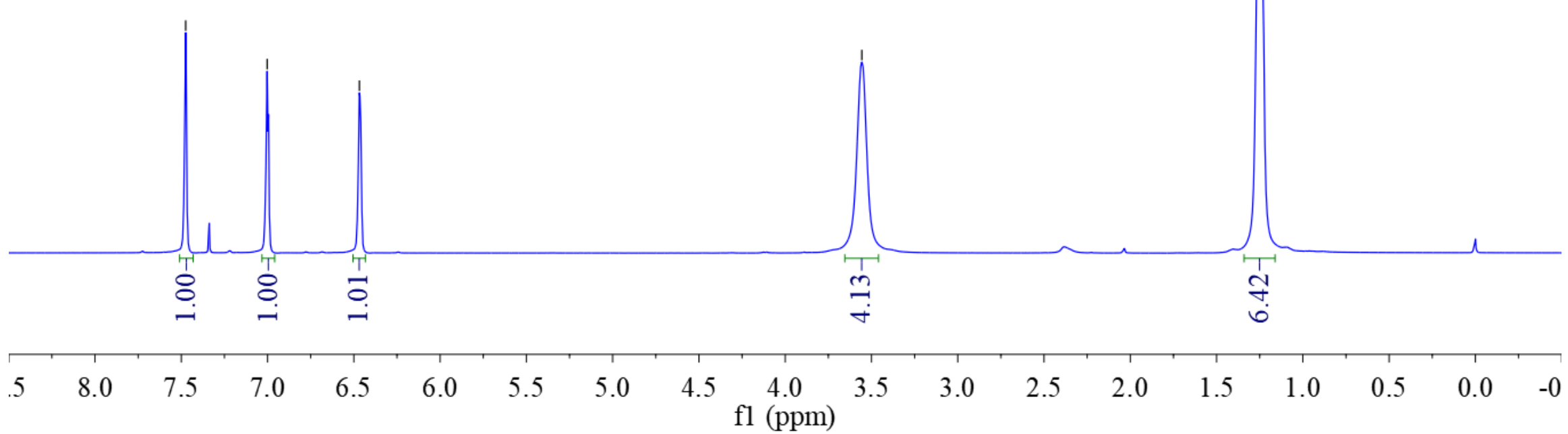


Figure S-6. ${ }^{13} \mathrm{C}\left\{{ }^{1} \mathrm{H}\right\}$ NMR spectrum of (2c) $\left(\mathrm{CDCl}_{3}, 100 \mathrm{MHz}\right) \boldsymbol{N}, \boldsymbol{N}$-diethylfuran-2-carboxamide
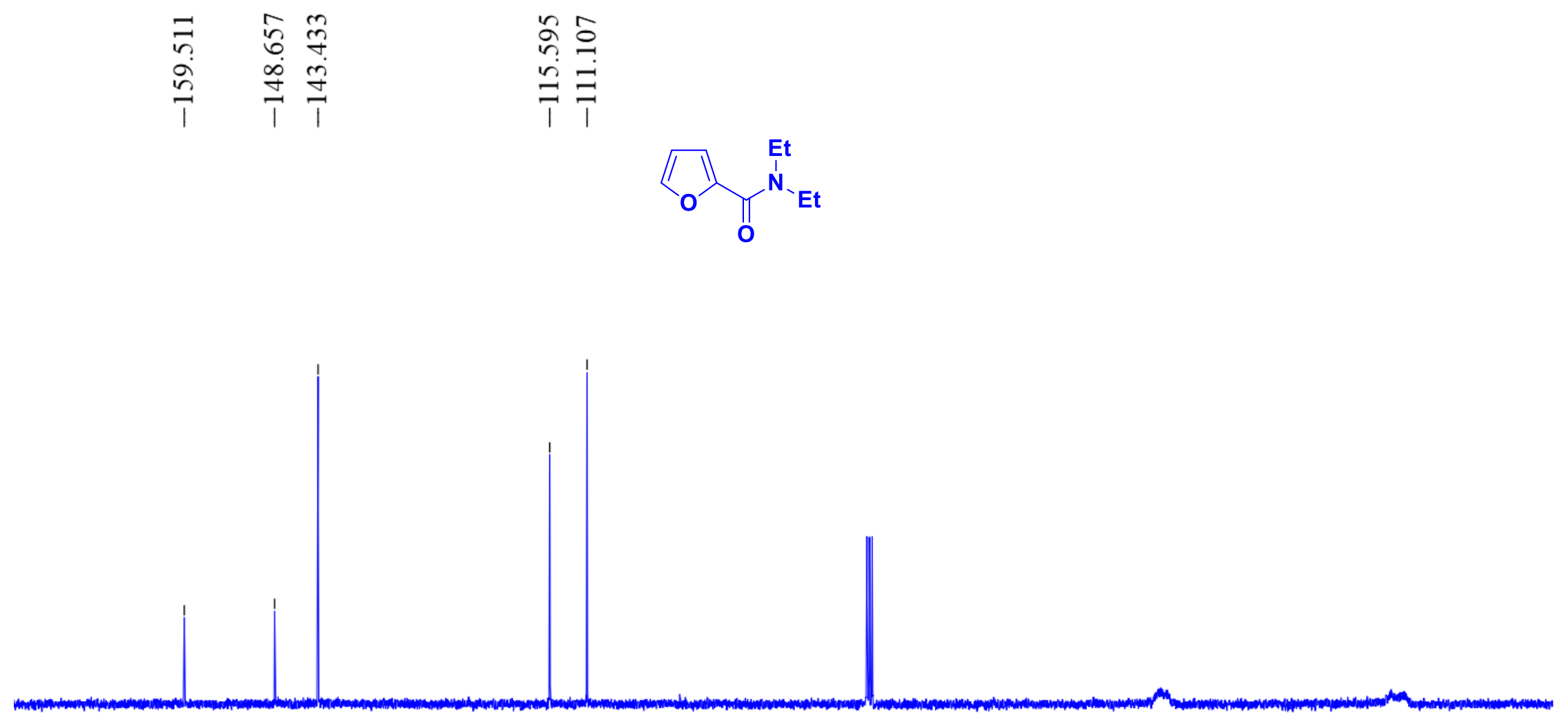

30

$170 \quad 160$

150

140

130

120

110

1009

fl $(\mathrm{ppm})$ 
Figure S-7. ${ }^{1} \mathrm{H}$ NMR spectrum of (2d) $\left(\mathrm{CDCl}_{3}, 400 \mathrm{MHz}\right) \mathrm{N}$-(2-bromophenyl)- $N$-methylfuran-2-carboxamide

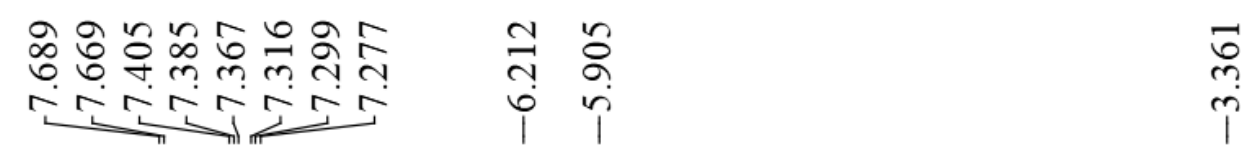

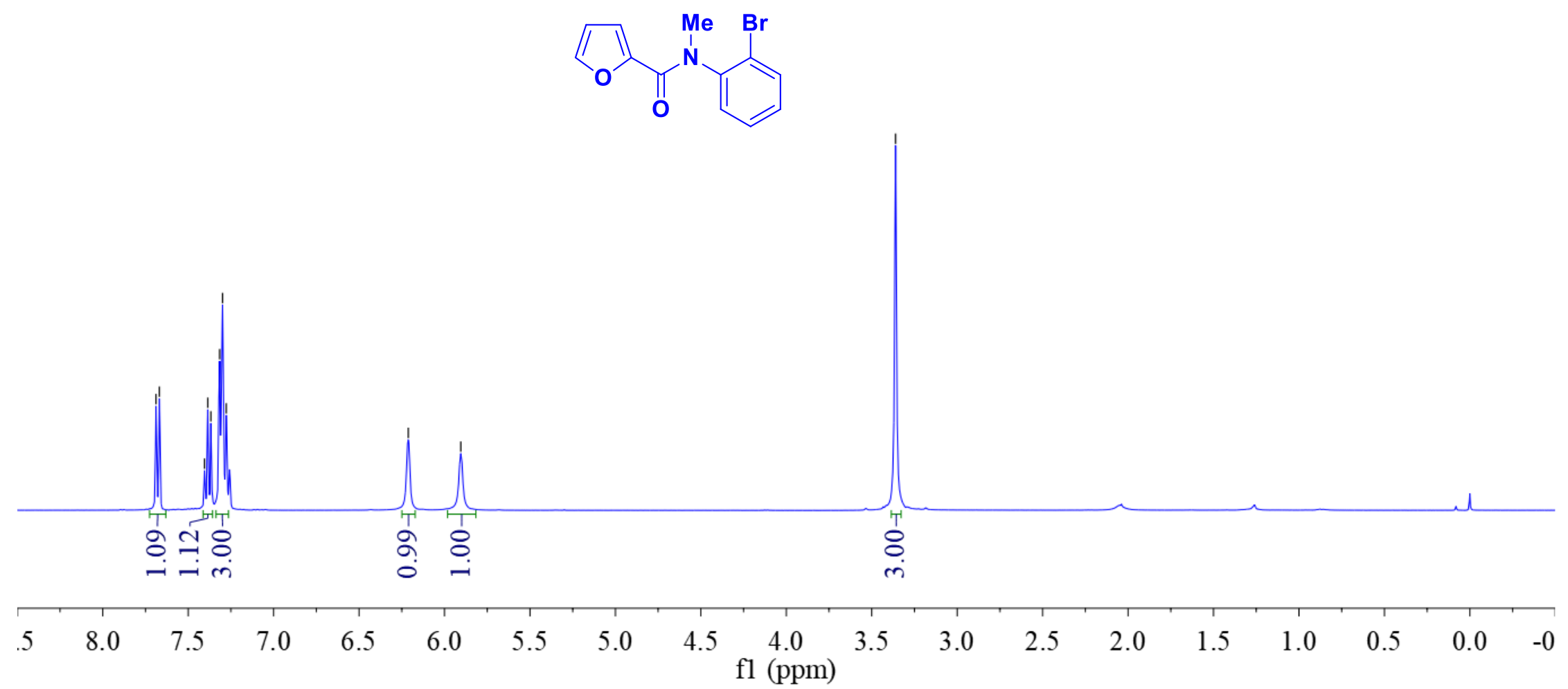


Figure S-8. ${ }^{13} \mathrm{C}\left\{{ }^{1} \mathrm{H}\right\}$ NMR spectrum of (2d) $\left(\mathrm{CDCl}_{3}, 100 \mathrm{MHz}\right) \mathbf{N}$-(2-bromophenyl)- $\boldsymbol{N}$-methylfuran-2-carboxamide

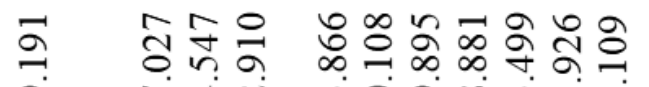

$$
\begin{aligned}
& \text { 俞 守吉 }
\end{aligned}
$$

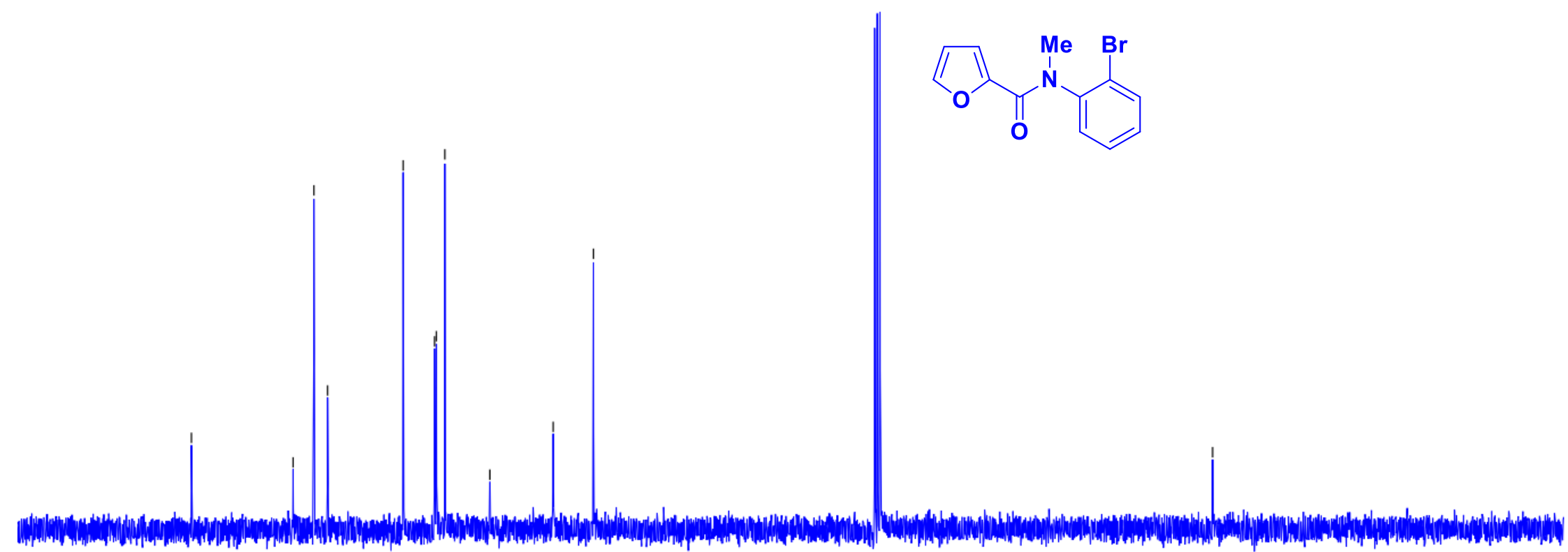

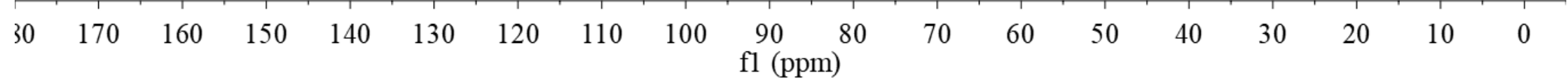


Figure S-9. ${ }^{1} \mathrm{H}$ NMR spectrum of (2e) $\left(\mathrm{CDCl}_{3}, 400 \mathrm{MHz}\right) \mathrm{N}$-(3-methoxyphenyl)- $N$-methylfuran-2-carboxamide

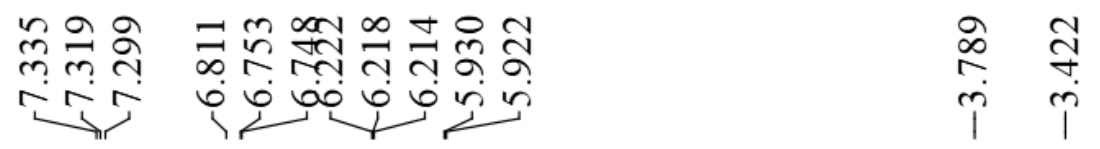

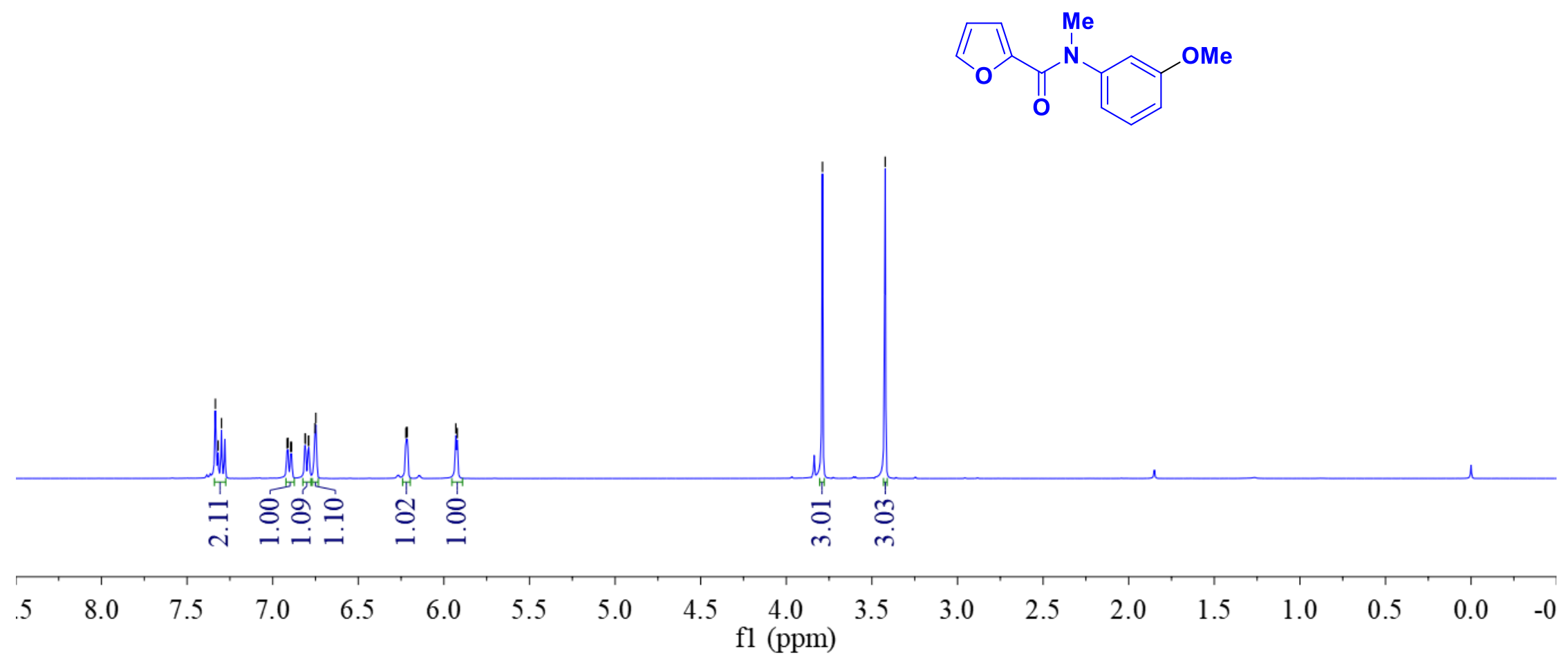


Figure S-10. ${ }^{13} \mathrm{C}\left\{{ }^{1} \mathrm{H}\right\}$ NMR spectrum of (2e) $\left(\mathrm{CDCl}_{3}, 100 \mathrm{MHz}\right) \boldsymbol{N}$-(3-methoxyphenyl)- $\boldsymbol{N}$-methylfuran-2-carboxamide

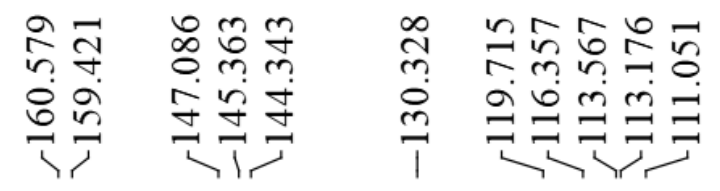
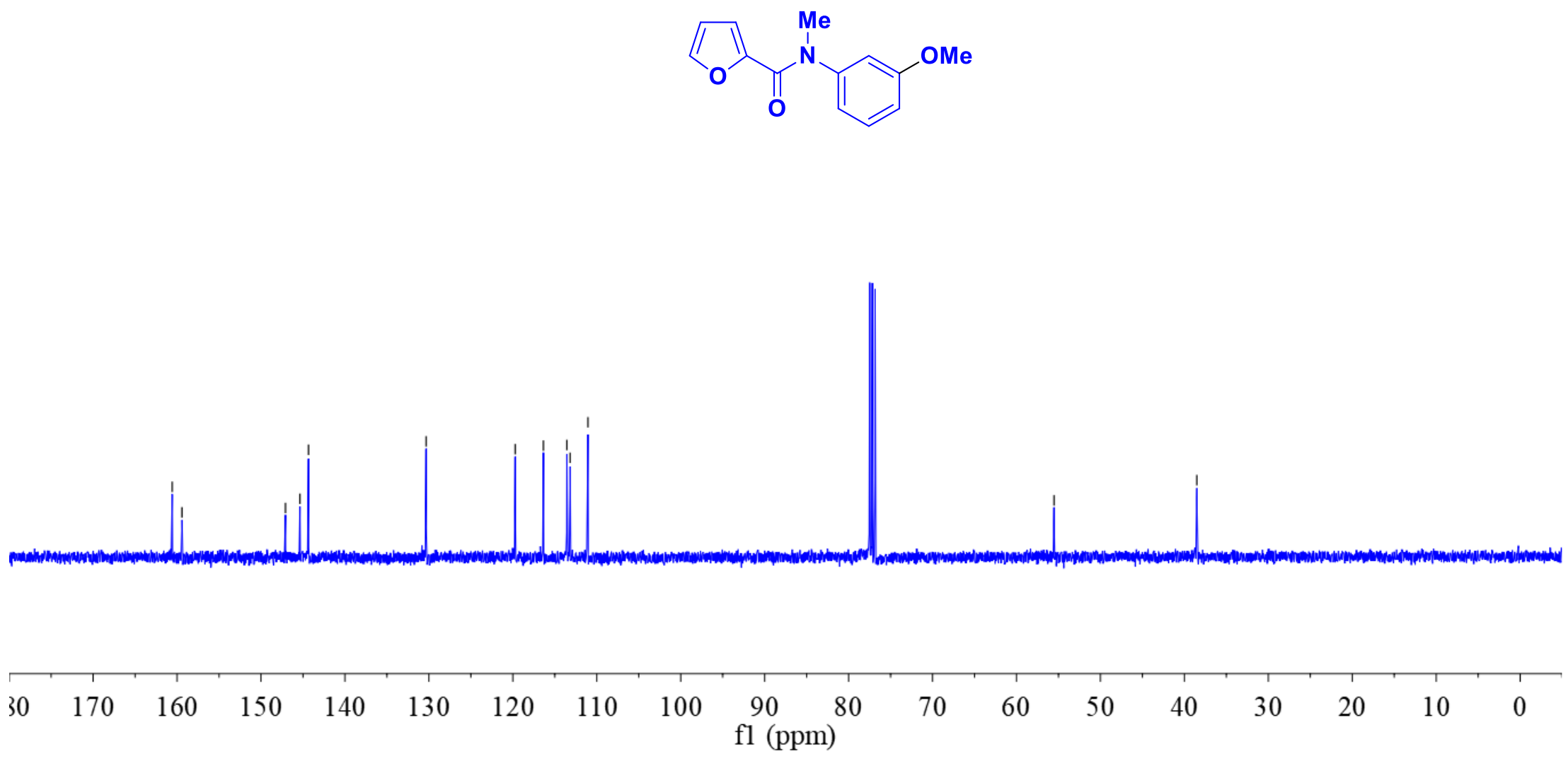
Figure S-11. ${ }^{1} \mathrm{H} \mathrm{NMR}$ spectrum of (2f) $\left(\mathrm{CDCl}_{3}, 400 \mathrm{MHz}\right)$ furan-2-yl(pyrrolidin-1-yl)methanone

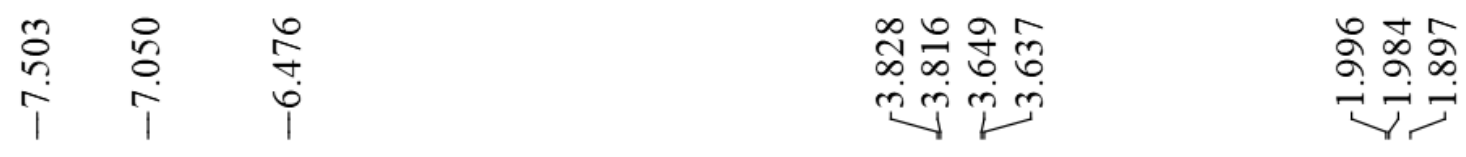
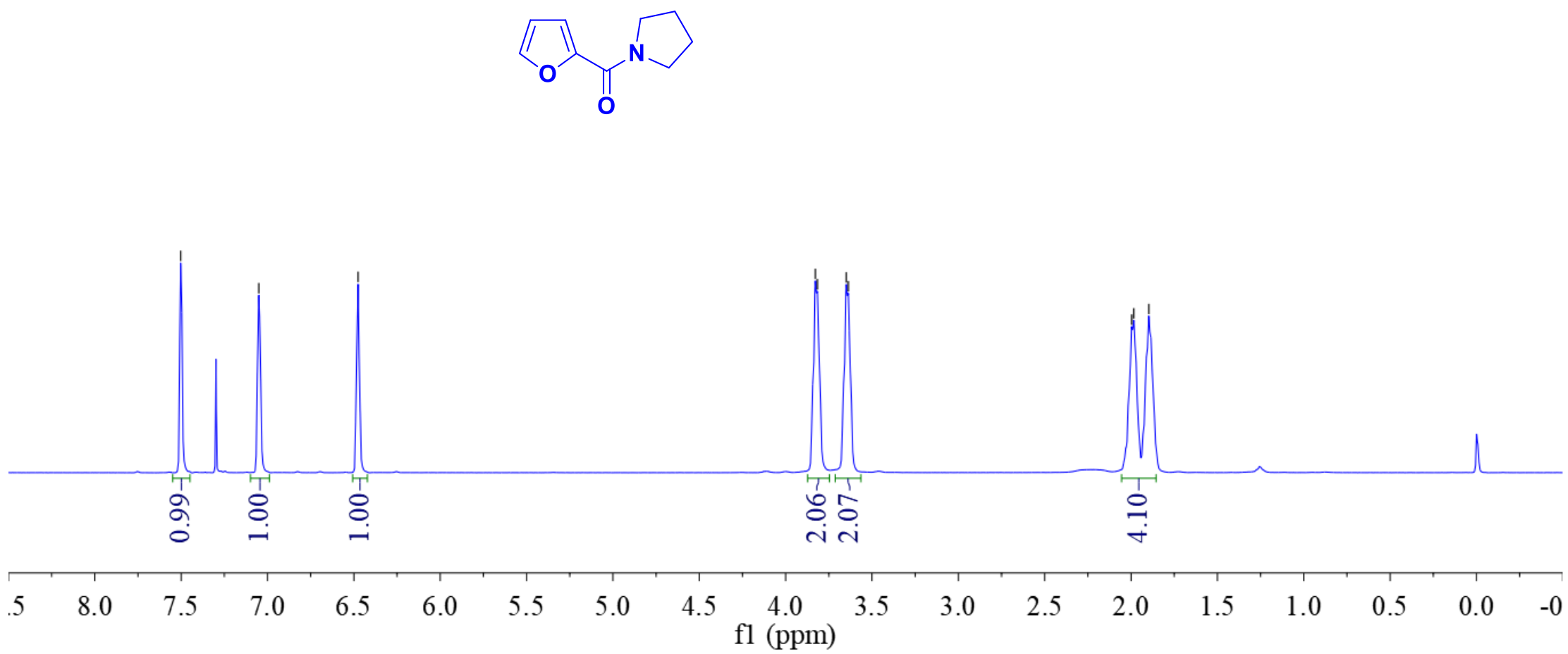
Figure S-12. ${ }^{13} \mathrm{C}\left\{{ }^{1} \mathrm{H}\right\}$ NMR spectrum of (2f) $\left(\mathrm{CDCl}_{3}, 100 \mathrm{MHz}\right)$ furan-2-yl(pyrrolidin-1-yl)methanone

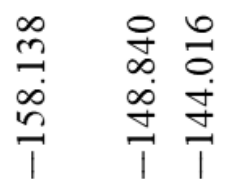

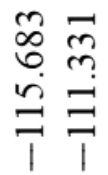

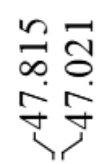$$
\rightarrow \frac{1}{2}
$$$$
\text { i }
$$<smiles>O=C(c1ccco1)N1CCCC1</smiles>

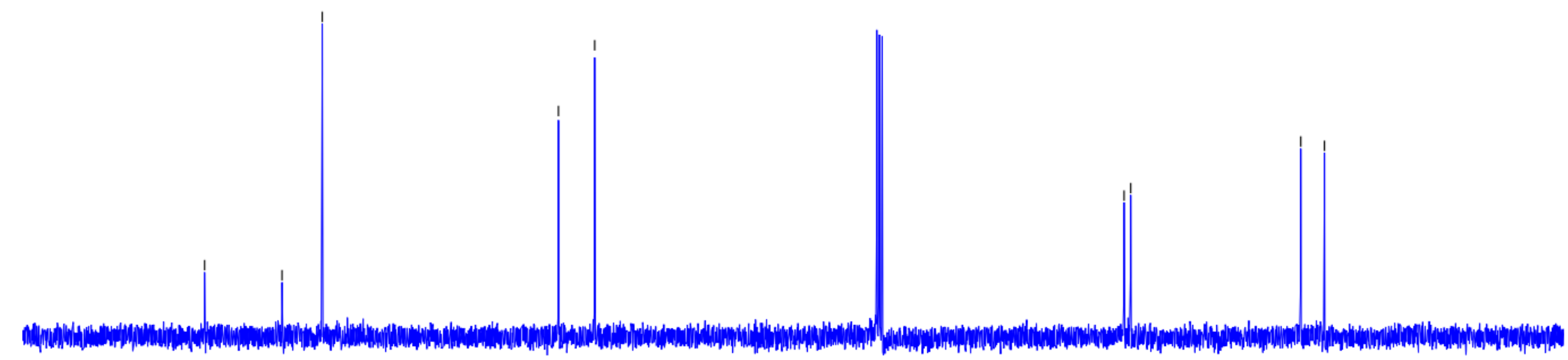

$30 \quad 170$

$150 \quad 140$

130

120

110

100

90

80

fl (ppm) 
Figure S-13. ${ }^{1} \mathrm{H}$ NMR spectrum of $(\mathbf{2 g})\left(\mathrm{CDCl}_{3}, 400 \mathrm{MHz}\right)$ furan-2-yl(piperidin-1-yl)methanone
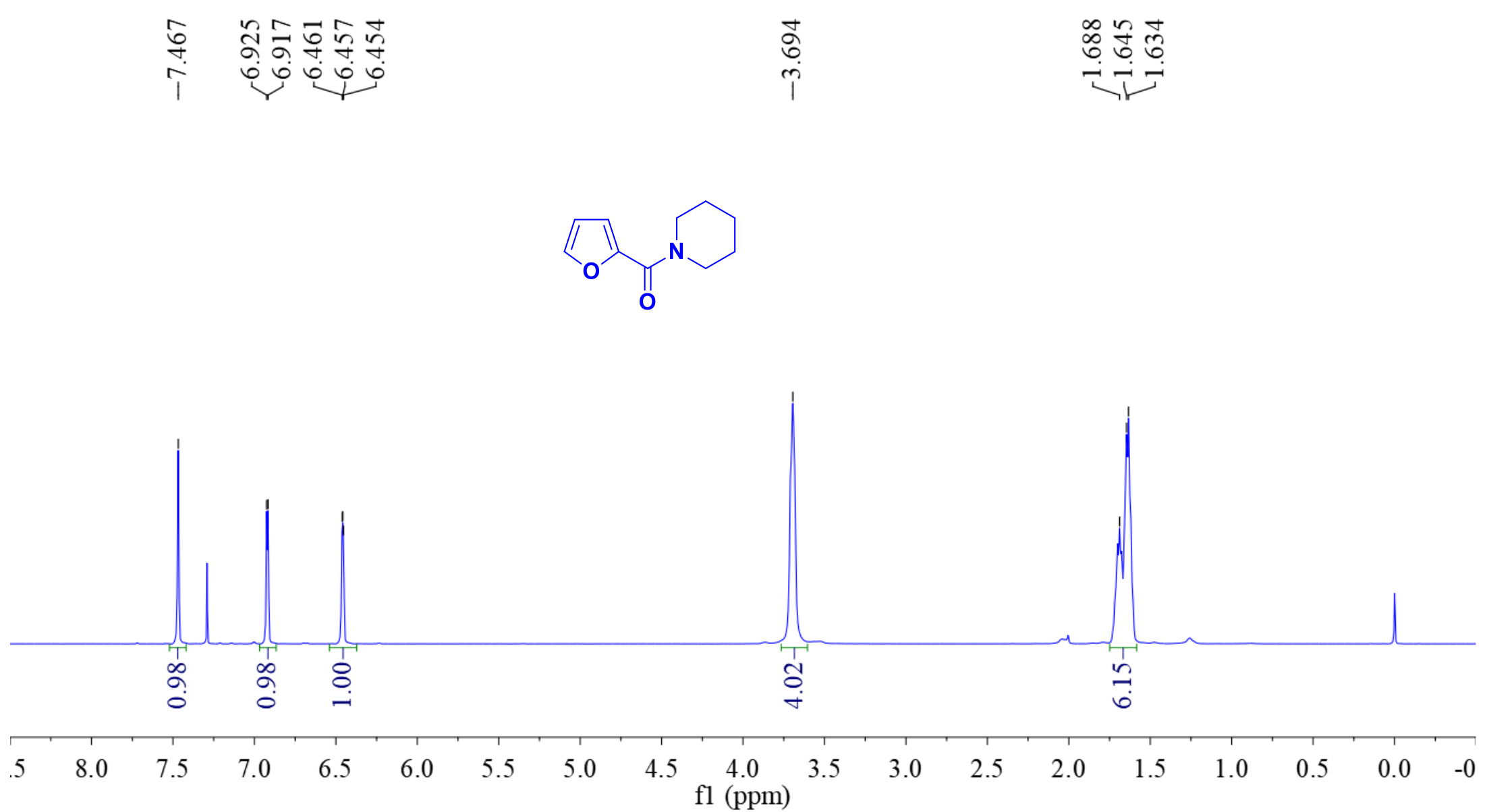
Figure S-14. ${ }^{13} \mathrm{C}\left\{{ }^{1} \mathrm{H}\right\}$ NMR spectrum of $(\mathbf{2 g})\left(\mathrm{CDCl}_{3}, 100 \mathrm{MHz}\right)$ furan-2-yl(piperidin-1-yl)methanone

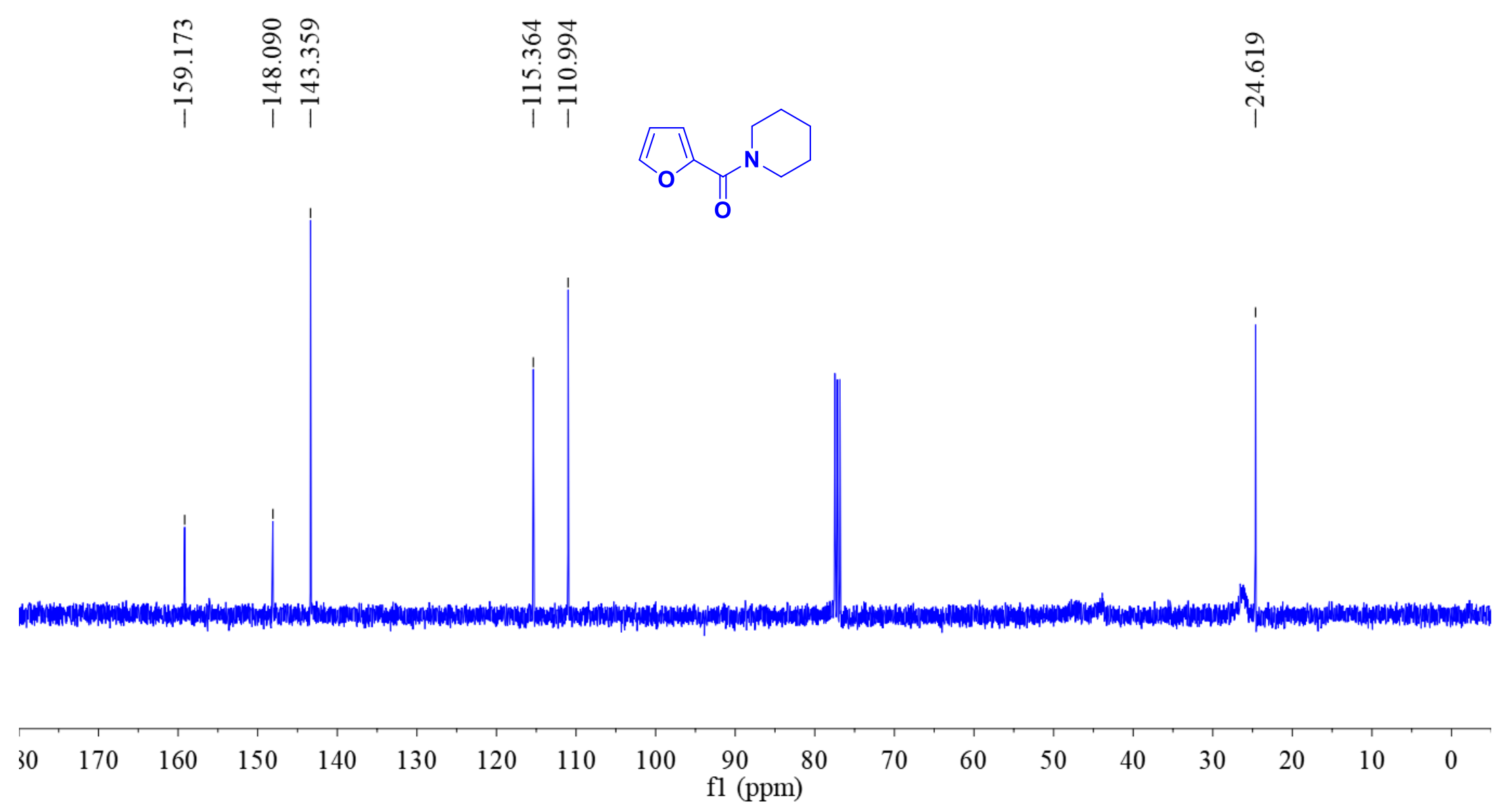


Figure S-15. ${ }^{1} \mathrm{H}$ NMR spectrum of (2h) $\left(\mathrm{CDCl}_{3}, 400 \mathrm{MHz}\right) \mathbf{N}$-methoxy- $\boldsymbol{N}$-methylfuran-2-carboxamide

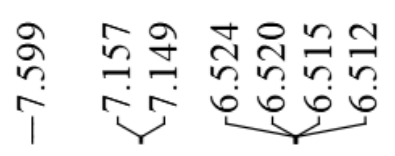

$\stackrel{i}{\stackrel{n}{r}} \stackrel{i}{i}$
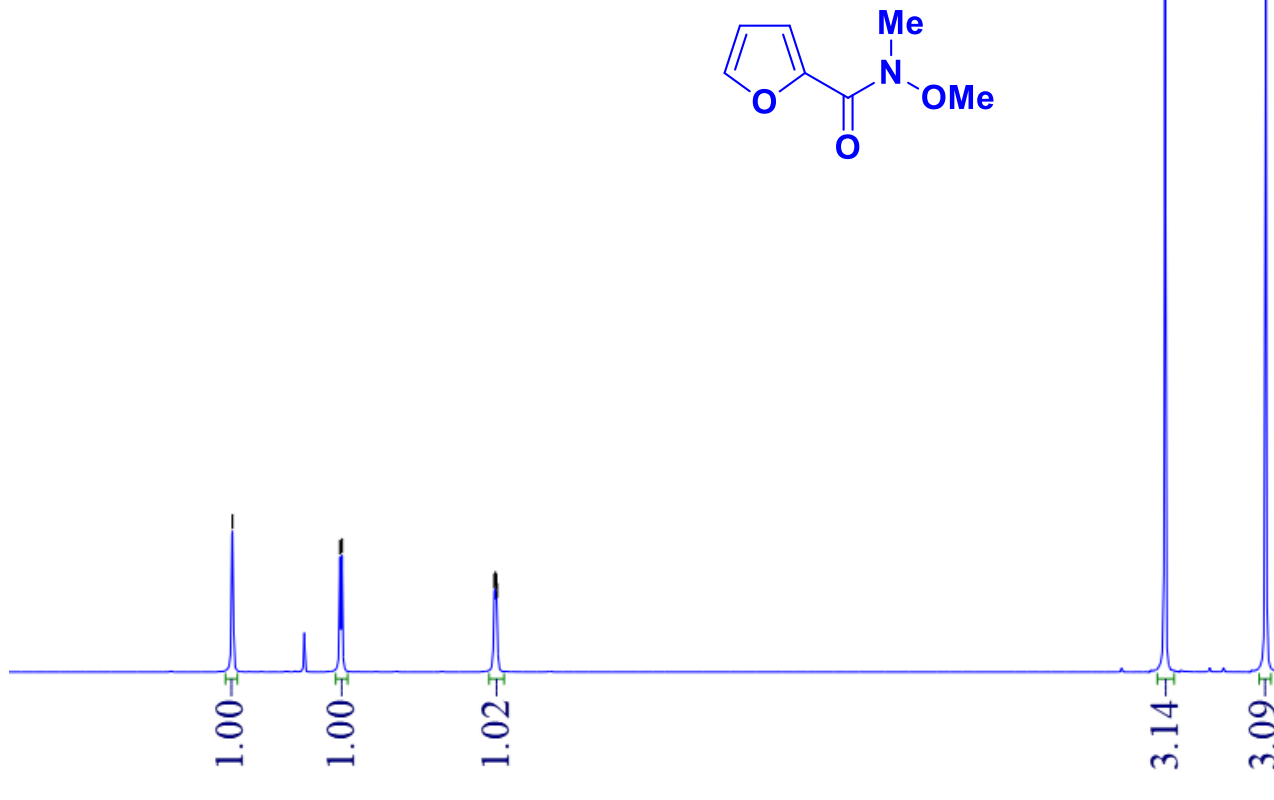

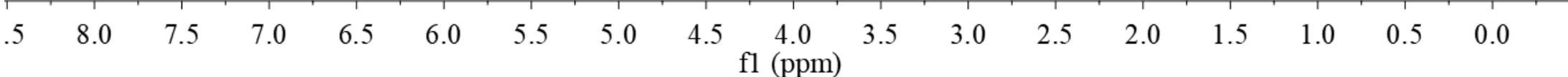


Figure S-16. ${ }^{13} \mathrm{C}\left\{{ }^{1} \mathrm{H}\right\}$ NMR spectrum of (2h) $\left(\mathrm{CDCl}_{3}, 100 \mathrm{MHz}\right) \boldsymbol{N}$-methoxy- $\boldsymbol{N}$-methylfuran-2-carboxamide

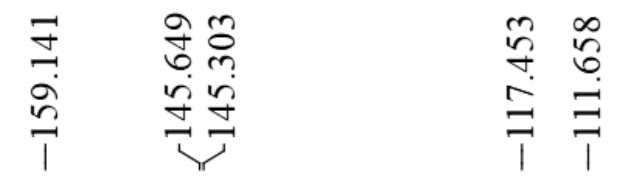

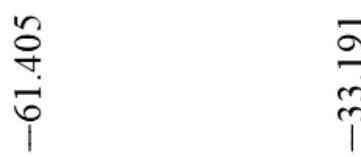
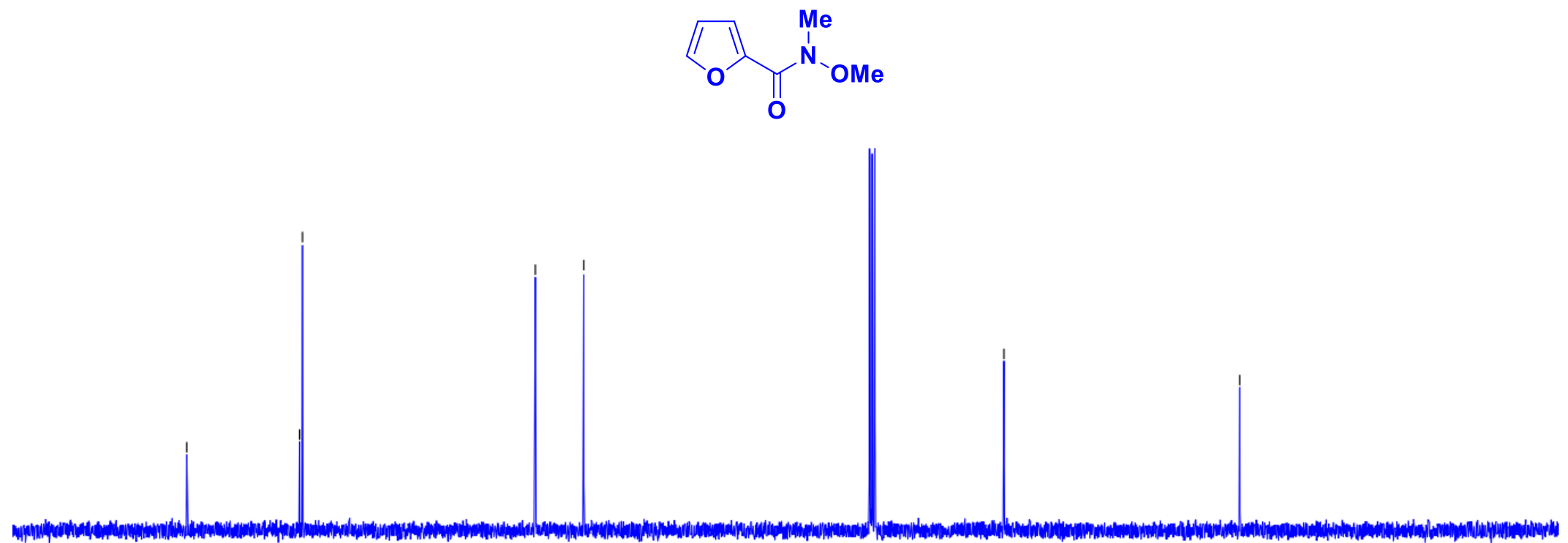

$30 \quad 170 \quad 160$

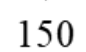

140

130

20

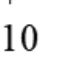

100

$90 \quad 80$

70 
Figure S-17. ${ }^{1} \mathrm{H}$ NMR spectrum of (2l) $\left(\mathrm{CDCl}_{3}, 400 \mathrm{MHz}\right)$ furan-2-yl(phenyl)methanone

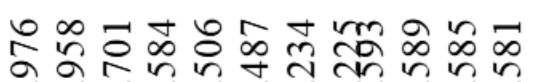

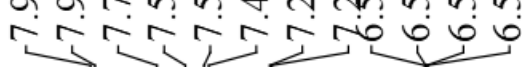
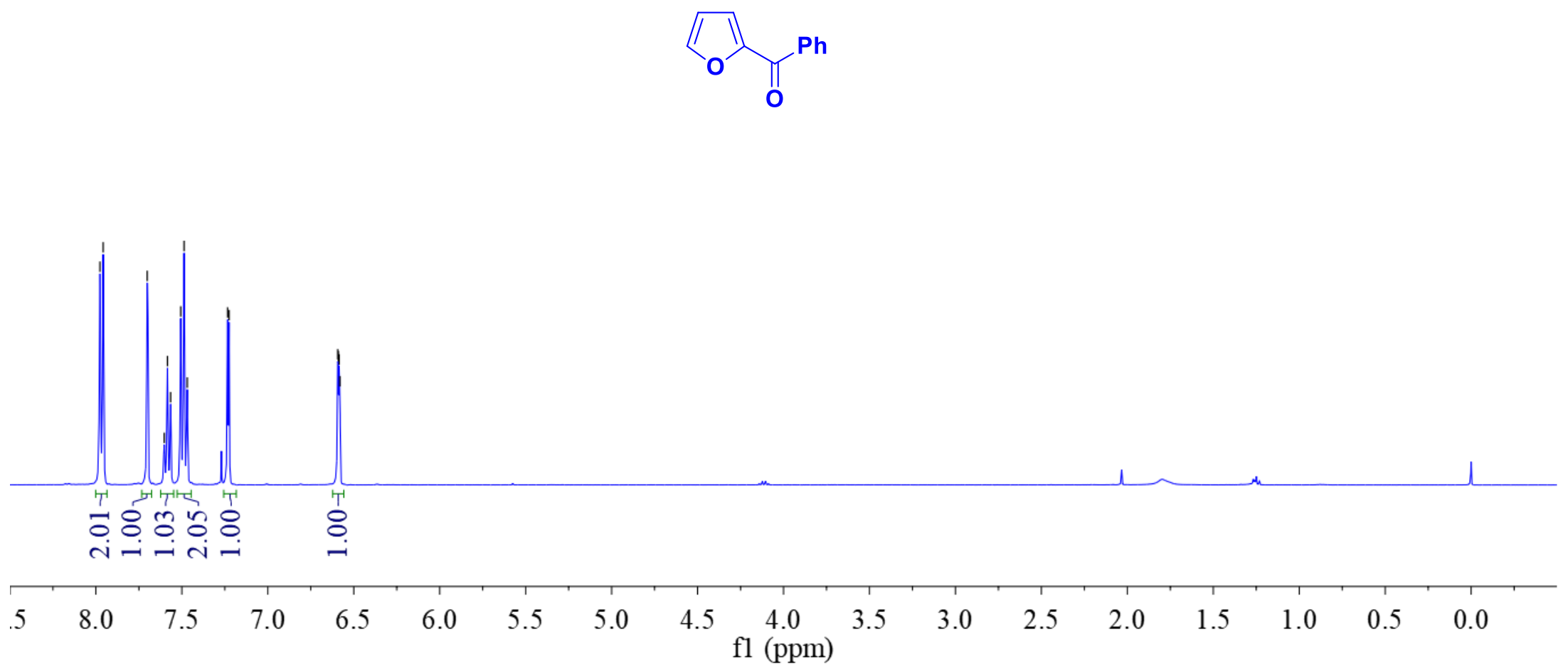
Figure S-18. ${ }^{13} \mathrm{C}\left\{{ }^{1} \mathrm{H}\right\}$ NMR spectrum of (2l) $\left(\mathrm{CDCl}_{3}, 100 \mathrm{MHz}\right)$ furan-2-yl(phenyl)methanone
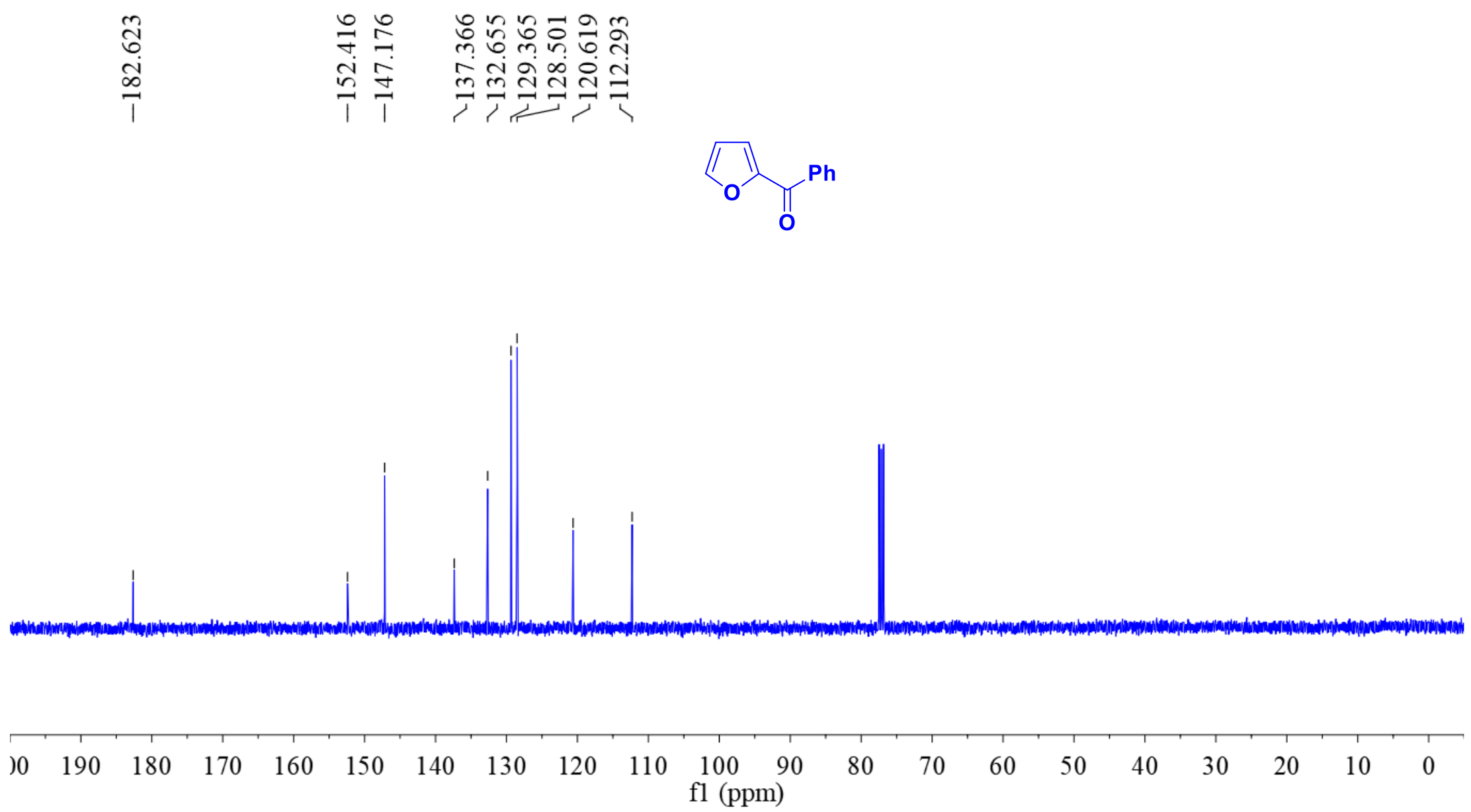
Figure S-19. ${ }^{1} \mathrm{H}$ NMR spectrum of (2o) $\left(\mathrm{CDCl}_{3}, 400 \mathrm{MHz}\right)$ 1-(furan-2-yl)-2,2-dimethylpropan-1-one

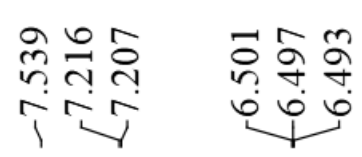

กั
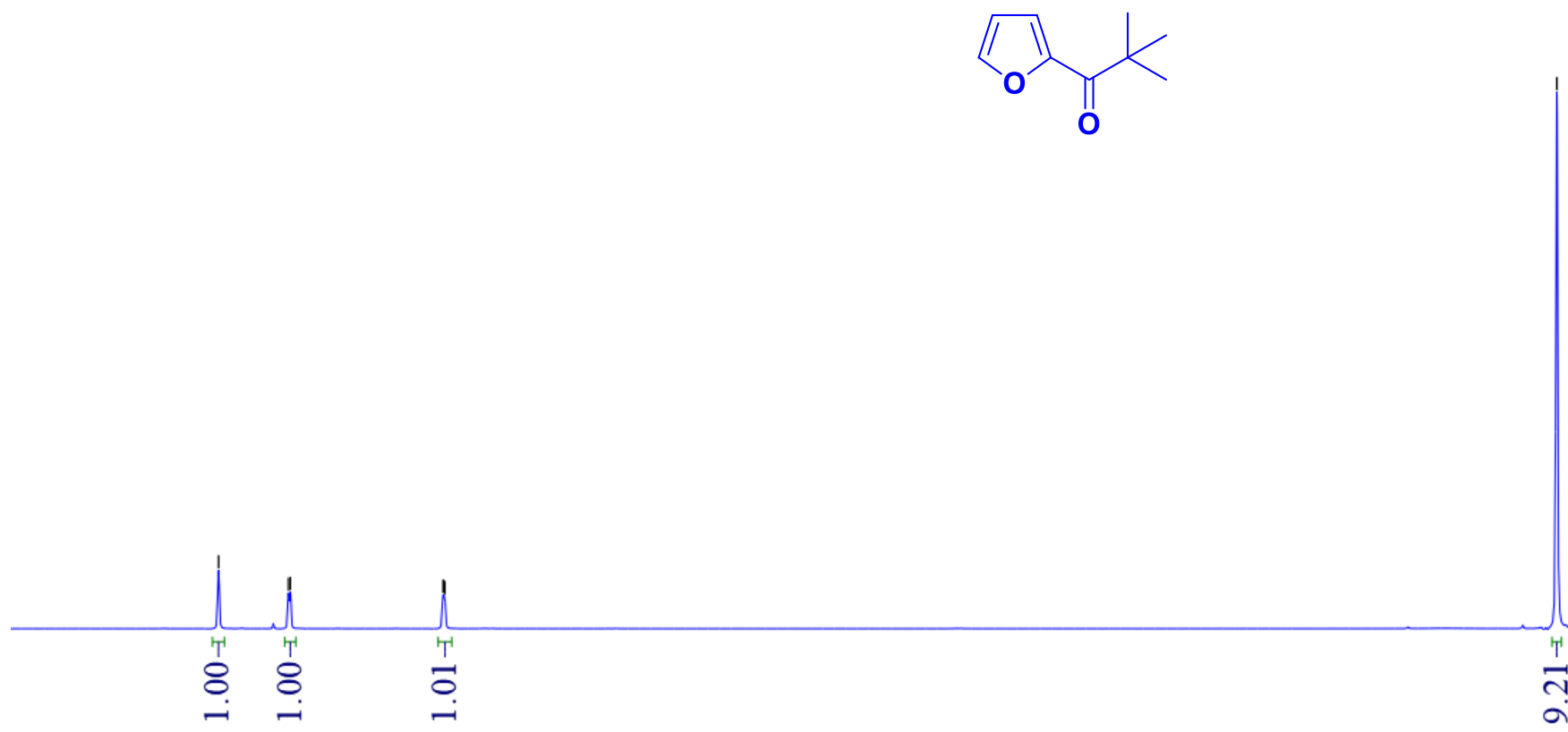

$\begin{array}{llllllllllllllllll}.5 & 8.0 & 7.5 & 7.0 & 6.5 & 6.0 & 5.5 & 5.0 & 4.5 \underset{\mathrm{fl}}{\underset{(\mathrm{ppm})}{4.0}} \mathbf{3 . 5} & 3.0 & 2.5 & 2.0 & 1.5 & 1.0 & 0.5 & 0.0 & -0\end{array}$

$\begin{array}{llllllllllllllllll}.5 & 8.0 & 7.5 & 7.0 & 6.5 & 6.0 & 5.5 & 5.0 & 4.5 \underset{\mathrm{fl}}{\mathrm{f}(\mathrm{ppm})} \mathbf{4 . 0} & 3.5 & 3.0 & 2.5 & 2.0 & 1.5 & 1.0 & 0.5 & 0.0 & -0\end{array}$

$\begin{array}{llllllllllllllllll}.5 & 8.0 & 7.5 & 7.0 & 6.5 & 6.0 & 5.5 & 5.0 & 4.5 \underset{\mathrm{fl}}{\mathrm{f( \textrm {ppm } )}} \mathbf{4 . 0} & 3.5 & 3.0 & 2.5 & 2.0 & 1.5 & 1.0 & 0.5 & 0.0 & -0\end{array}$

$\stackrel{\substack{r \\ i}}{i}$

$\frac{T}{2}$ 
Figure S-20. ${ }^{13} \mathrm{C}\left\{{ }^{1} \mathrm{H}\right\}$ NMR spectrum of (2o) $\left(\mathrm{CDCl}_{3}, 100 \mathrm{MHz}\right)$ 1-(furan-2-yl)-2,2-dimethylpropan-1-one

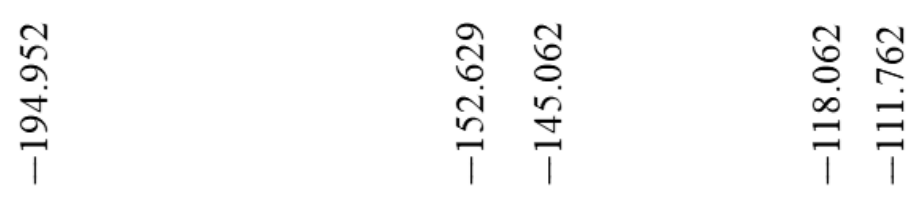

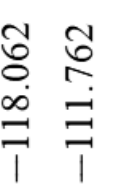
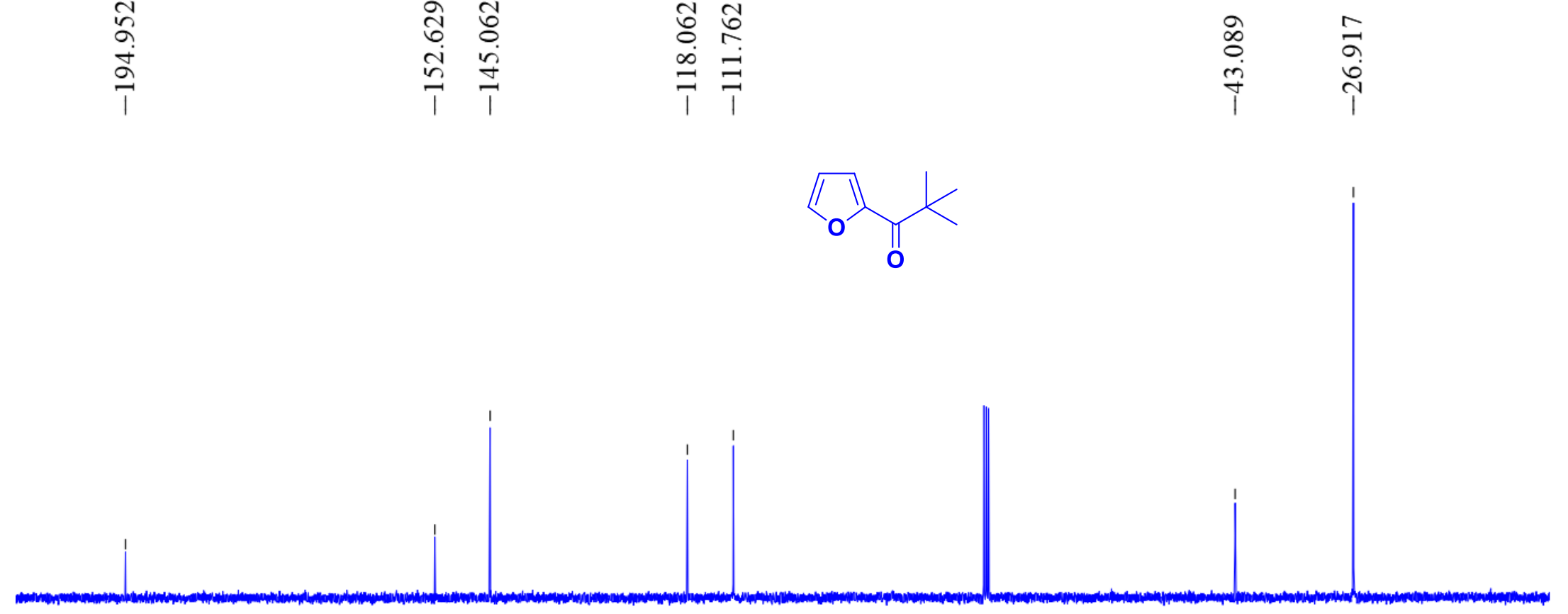

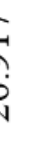

$\begin{array}{lllllllllllllllllllll}10 & 200 & 190 & 180 & 170 & 160 & 150 & 140 & 130 & 120 & \begin{array}{c}110 \\ \mathrm{fl}(\mathrm{ppm})\end{array} & 90 & 80 & 70 & 60 & 50 & 40 & 30 & 20 & 10 & (\end{array}$ 
Figure S-21. ${ }^{1} \mathrm{H}$ NMR spectrum of (2p) $\left(\mathrm{CDCl}_{3}, 400 \mathrm{MHz}\right)$ 2-phenylfuran

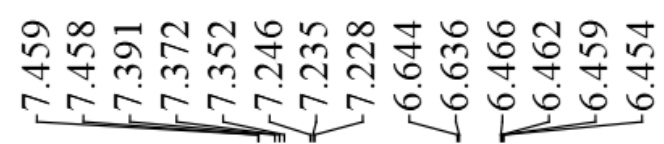
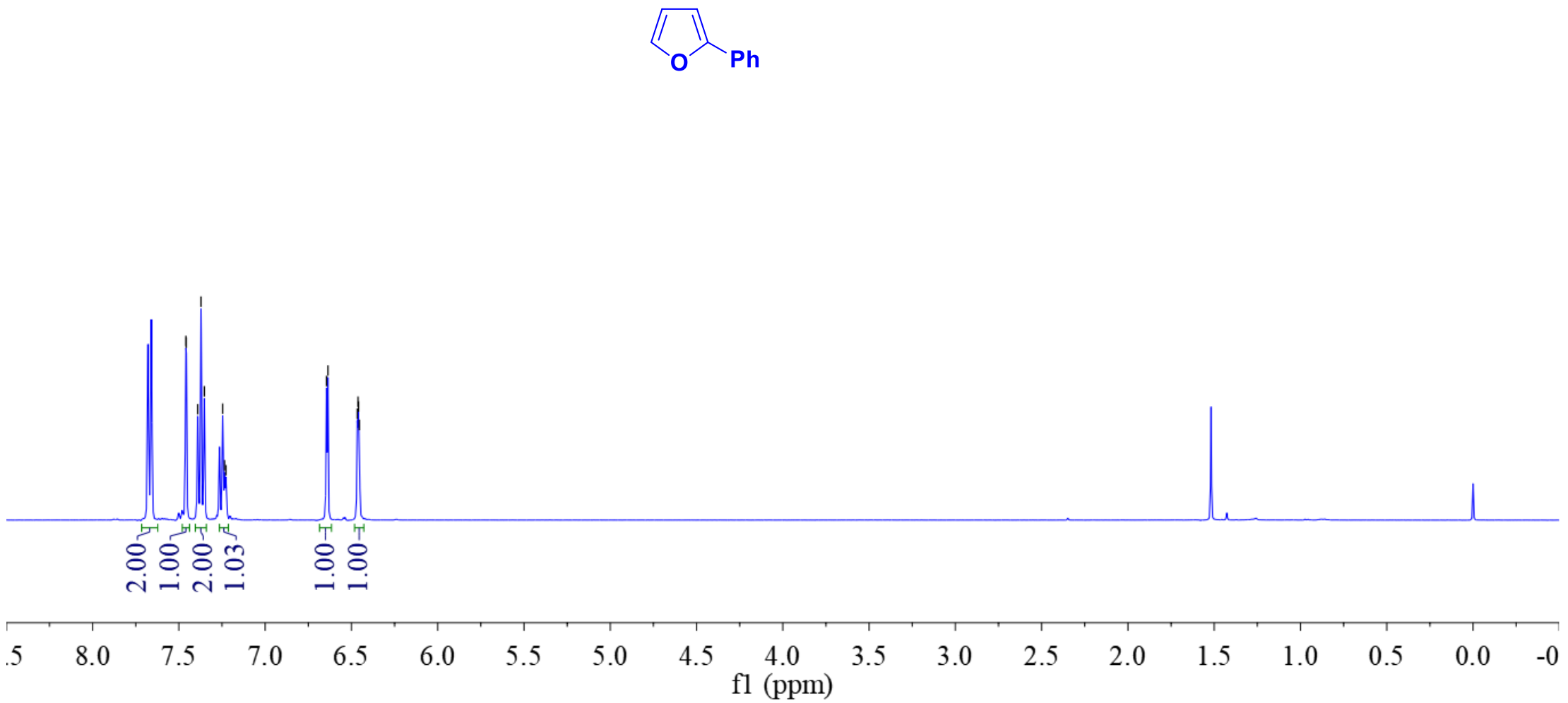
Figure S-22. ${ }^{13} \mathrm{C}\left\{{ }^{1} \mathrm{H}\right\}$ NMR spectrum of (2p) $\left(\mathrm{CDCl}_{3}, 100 \mathrm{MHz}\right)$ 2-phenylfuran

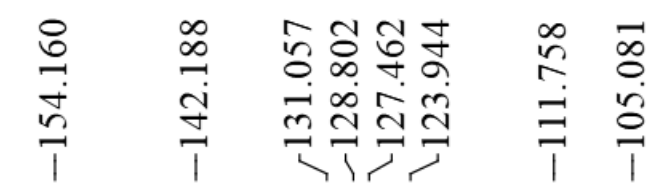
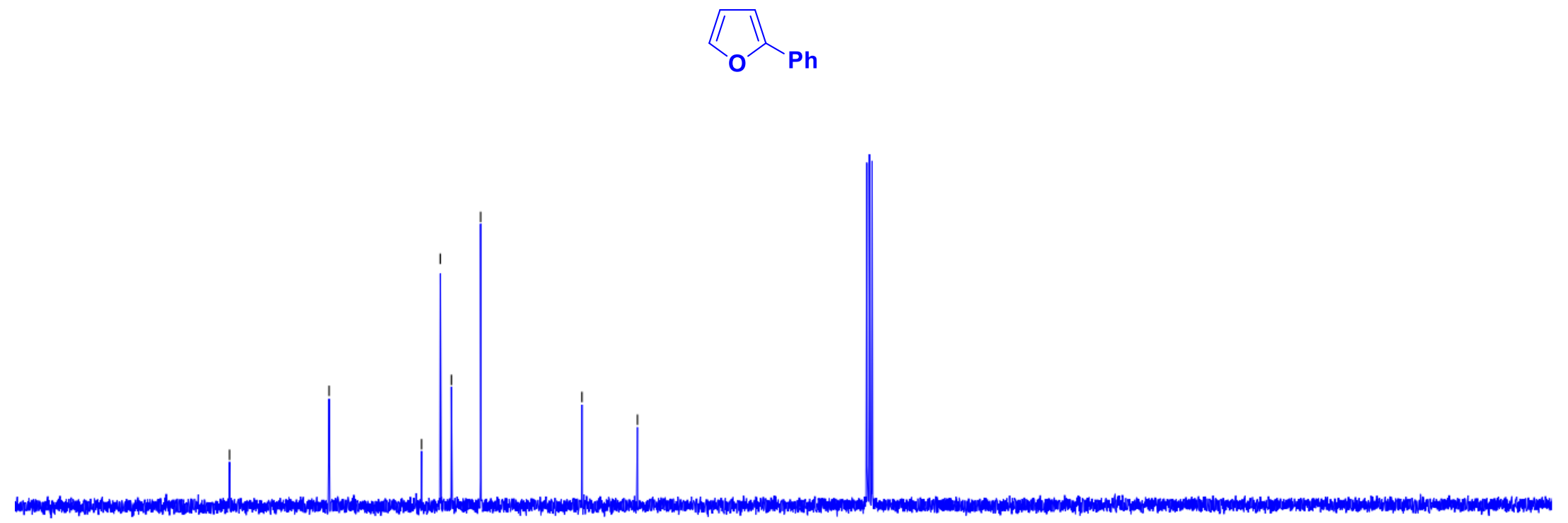

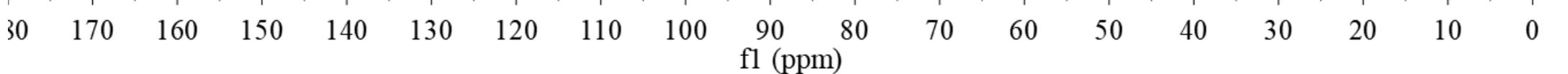


Figure S-23. ${ }^{1} \mathrm{H}$ NMR spectrum of $(2 \mathrm{~s})\left(\mathrm{CDCl}_{3}, 400 \mathrm{MHz}\right) \boldsymbol{N}$-methyl- $\boldsymbol{N}$-phenylfuran-3-carboxamide

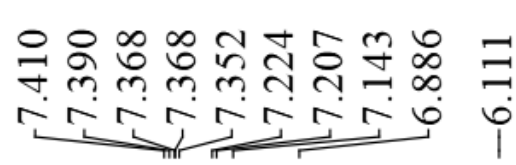

$\stackrel{0}{\underset{1}{*}}$

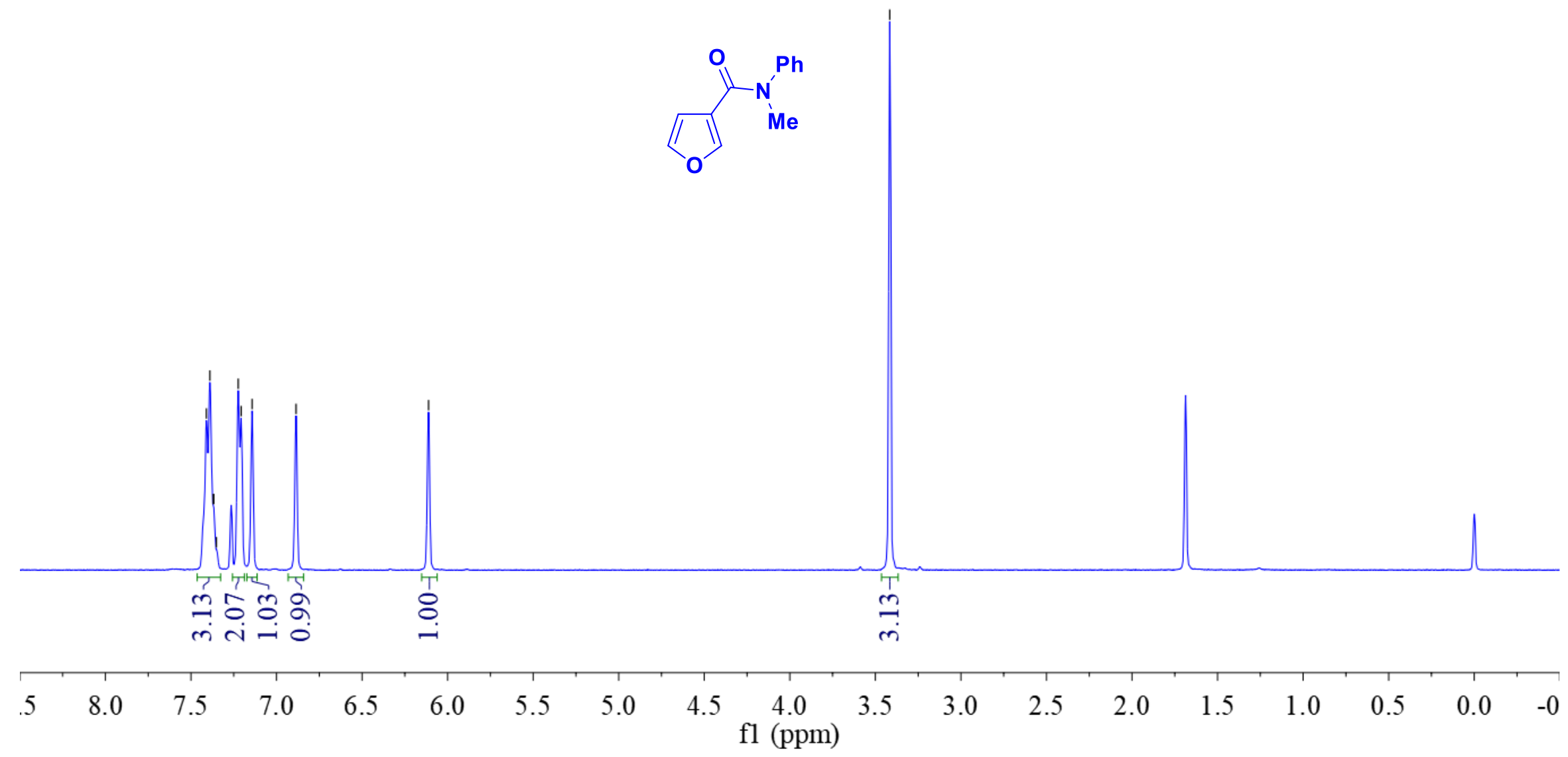


Figure S-24. ${ }^{13} \mathrm{C}\left\{{ }^{1} \mathrm{H}\right\}$ NMR spectrum of (2s) $\left(\mathrm{CDCl}_{3}, 100 \mathrm{MHz}\right) \boldsymbol{N}$-methyl- $\boldsymbol{N}$-phenylfuran-3-carboxamide

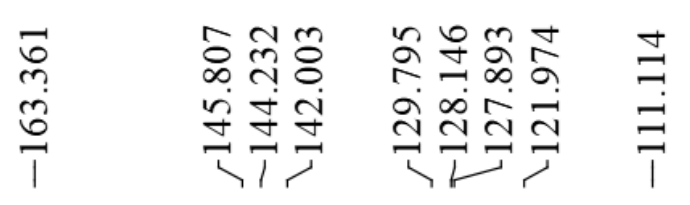
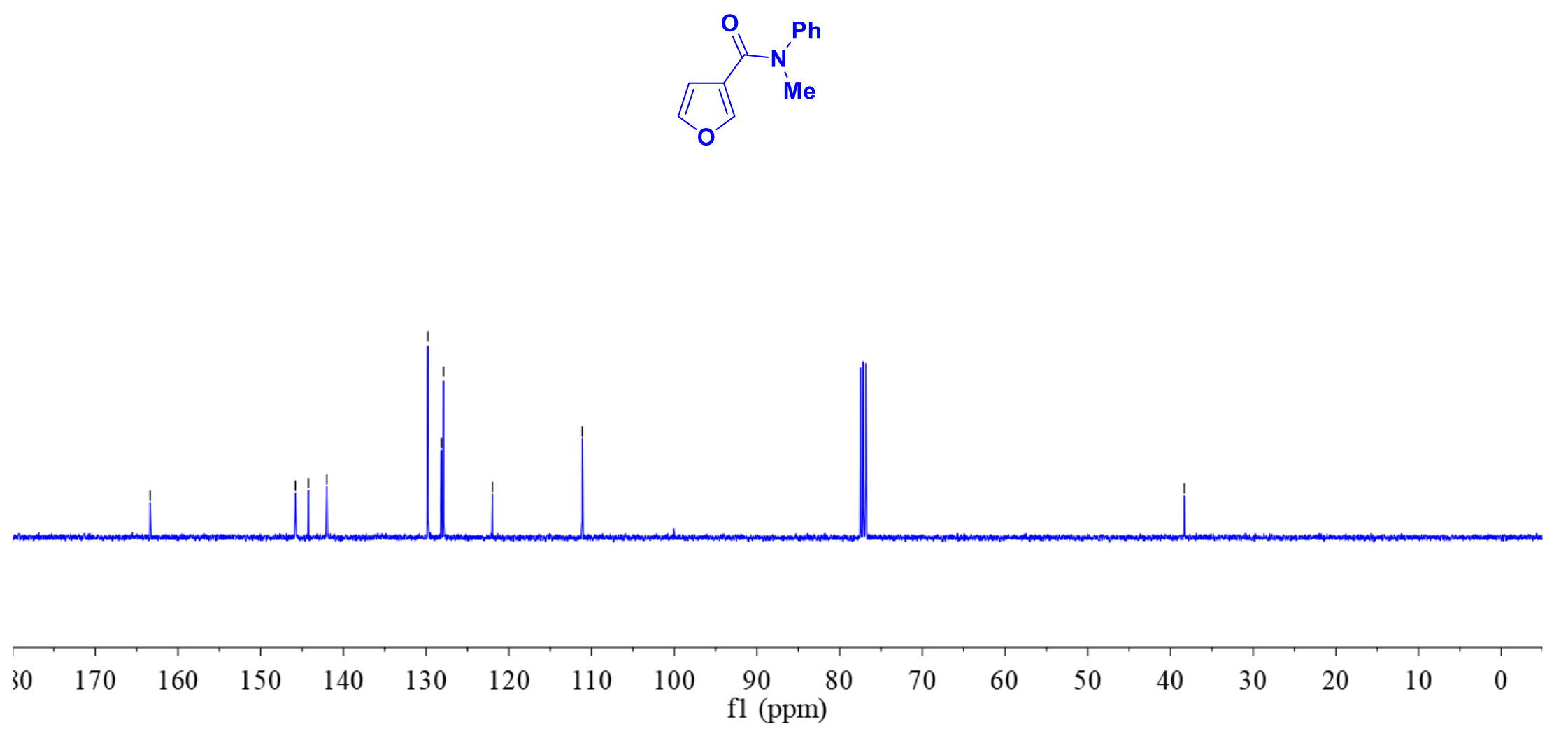
Figure S-25. ${ }^{1} \mathrm{H}$ NMR spectrum of (3c) $\left(\mathrm{CDCl}_{3}, 400 \mathrm{MHz}\right)$ 1-methylcyclohexanecarboxaldehyde
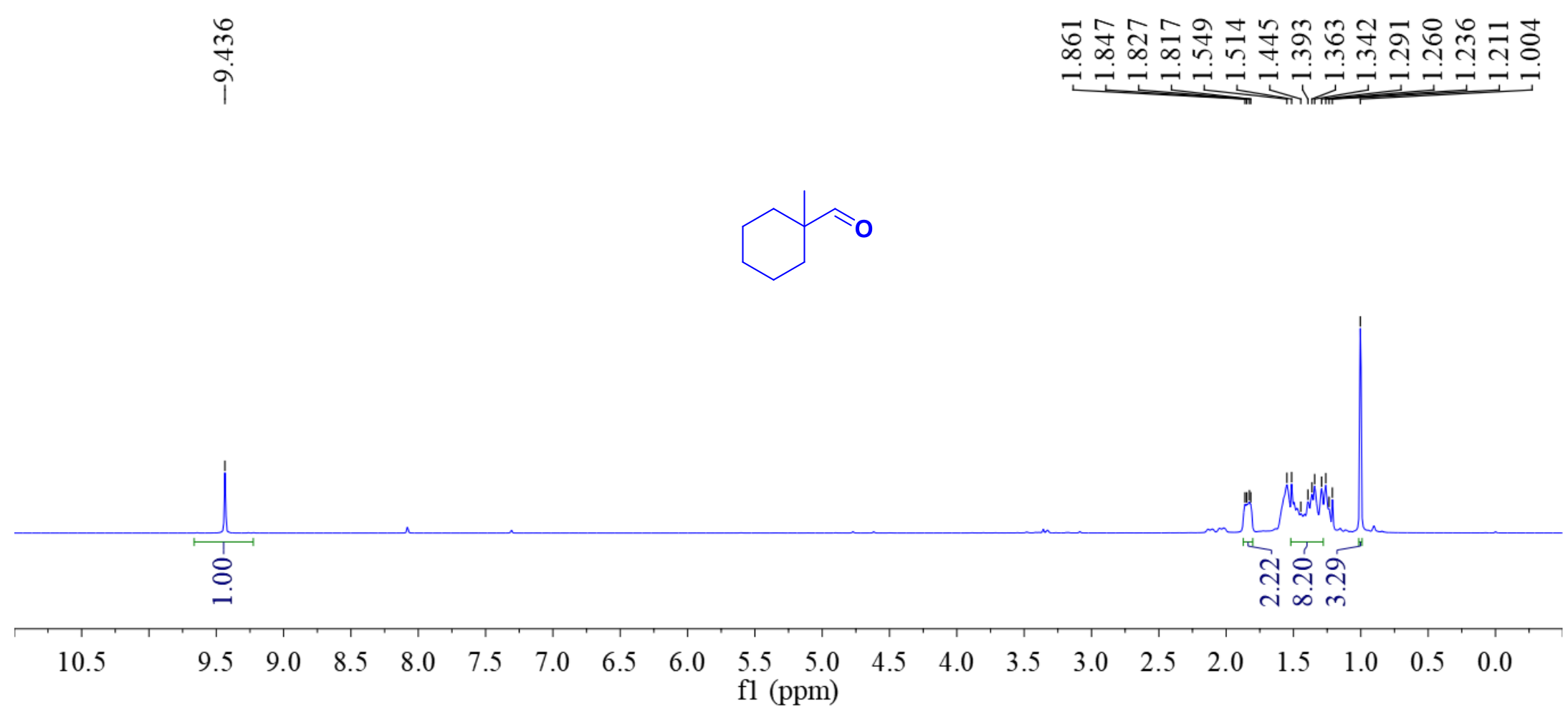
Figure S-26. ${ }^{1} \mathrm{H}$ NMR spectrum of (1a) $\left(\mathrm{CDCl}_{3}, 400 \mathrm{MHz}\right)$ 5-(tert-butyl)- $\boldsymbol{N}$-methyl- $\boldsymbol{N}$-phenylfuran-2-carboxamide

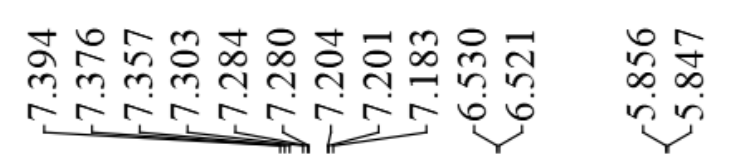
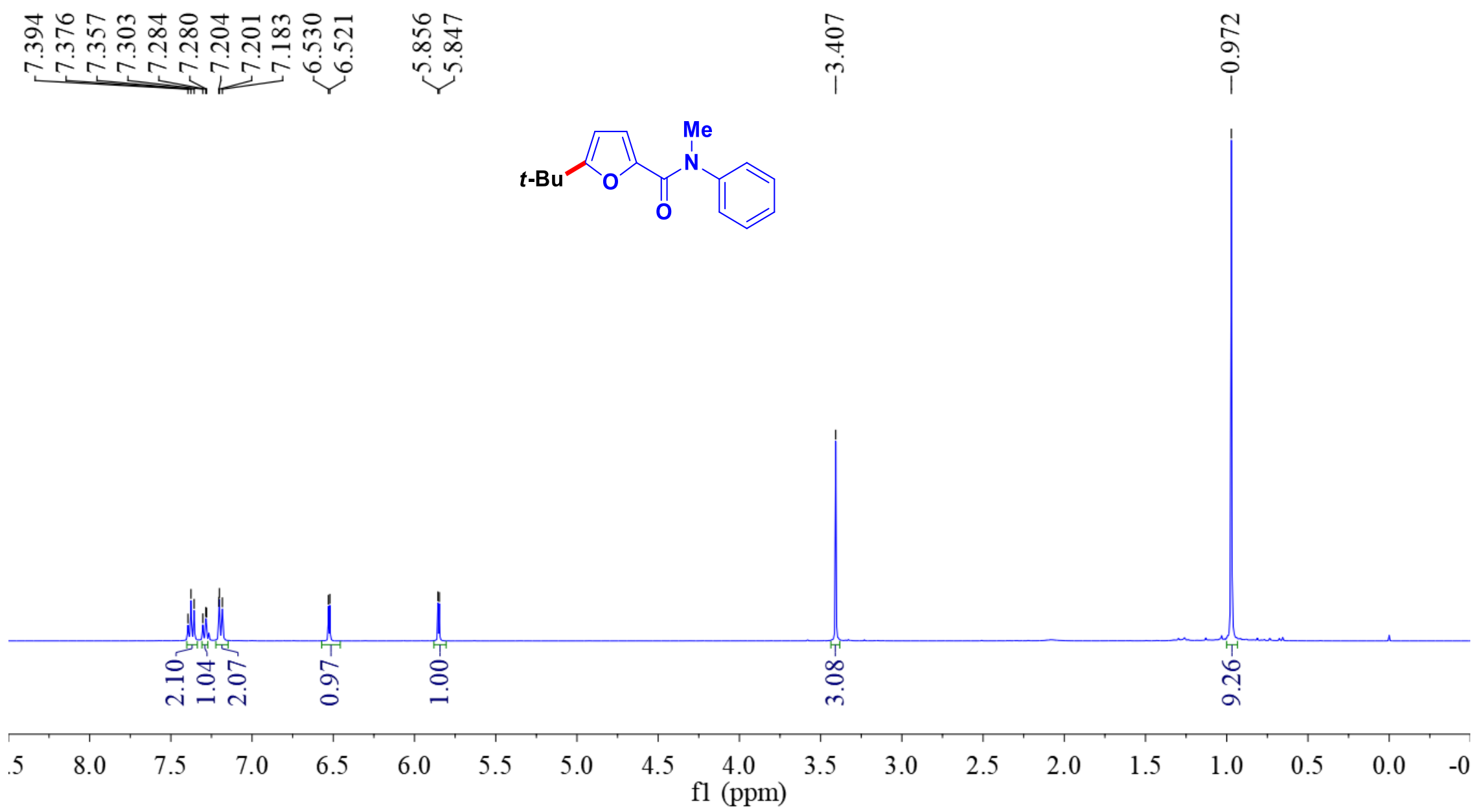
Figure S-27. ${ }^{13} \mathrm{C}\left\{{ }^{1} \mathrm{H}\right\}$ NMR spectrum of (1a) $\left(\mathrm{CDCl}_{3}, 100 \mathrm{MHz}\right)$ 5-(tert-butyl)- $\boldsymbol{N}$-methyl- $\boldsymbol{N}$-phenylfuran-2-carboxamide

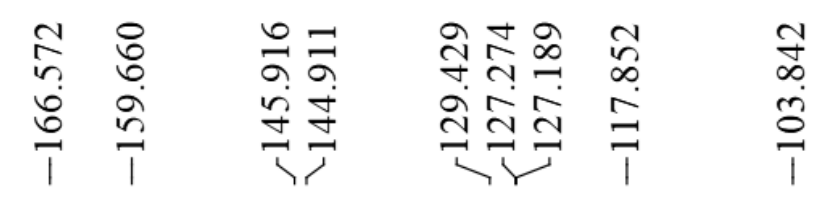

$$
\begin{aligned}
& \text { ㅎํ } \\
& \text { ஸึ ํํ }
\end{aligned}
$$
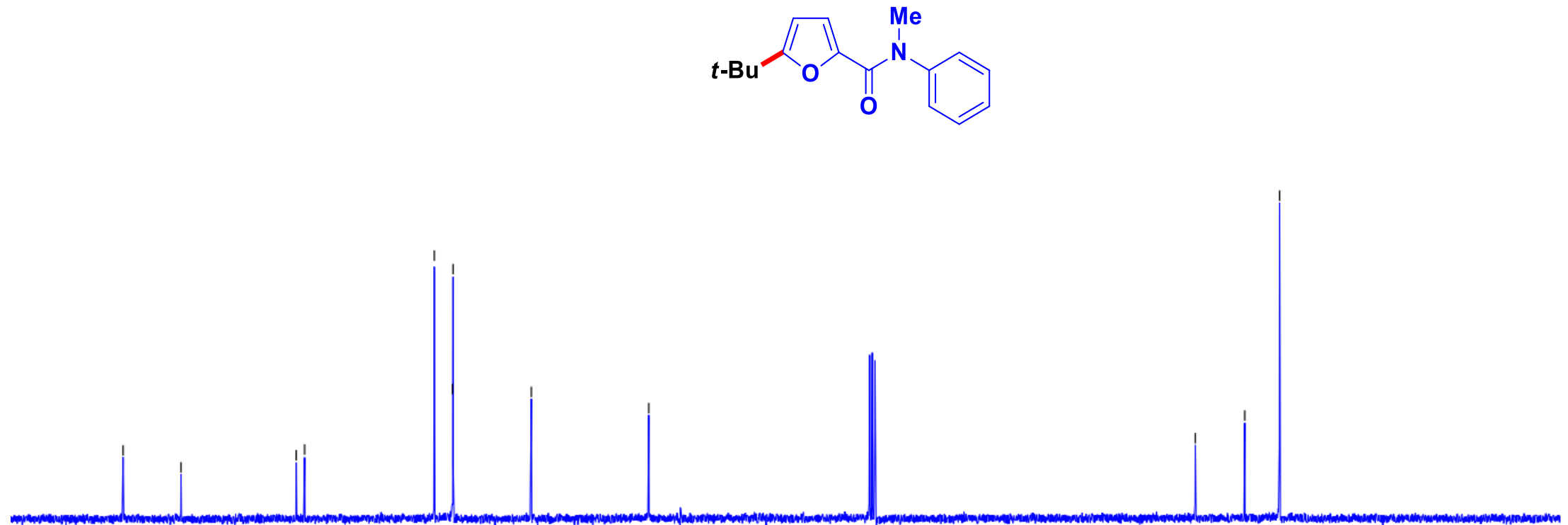

$30 \quad 170$

160

$150 \quad 140$

130

120

110

10090

9080

$70 \quad 60$

$50 \quad 40$

$40 \quad 30$

$20 \quad 10 \quad 0$


Figure S-28. ${ }^{1} \mathrm{H}$ NMR spectrum of (1b) $\left(\mathrm{CDCl}_{3}, 400 \mathrm{MHz}\right)$ 5-(tert-butyl)- $N$, $N$-dimethylfuran-2-carboxamide

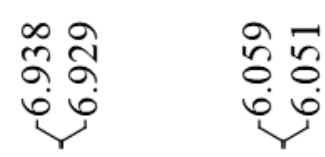
थั๊

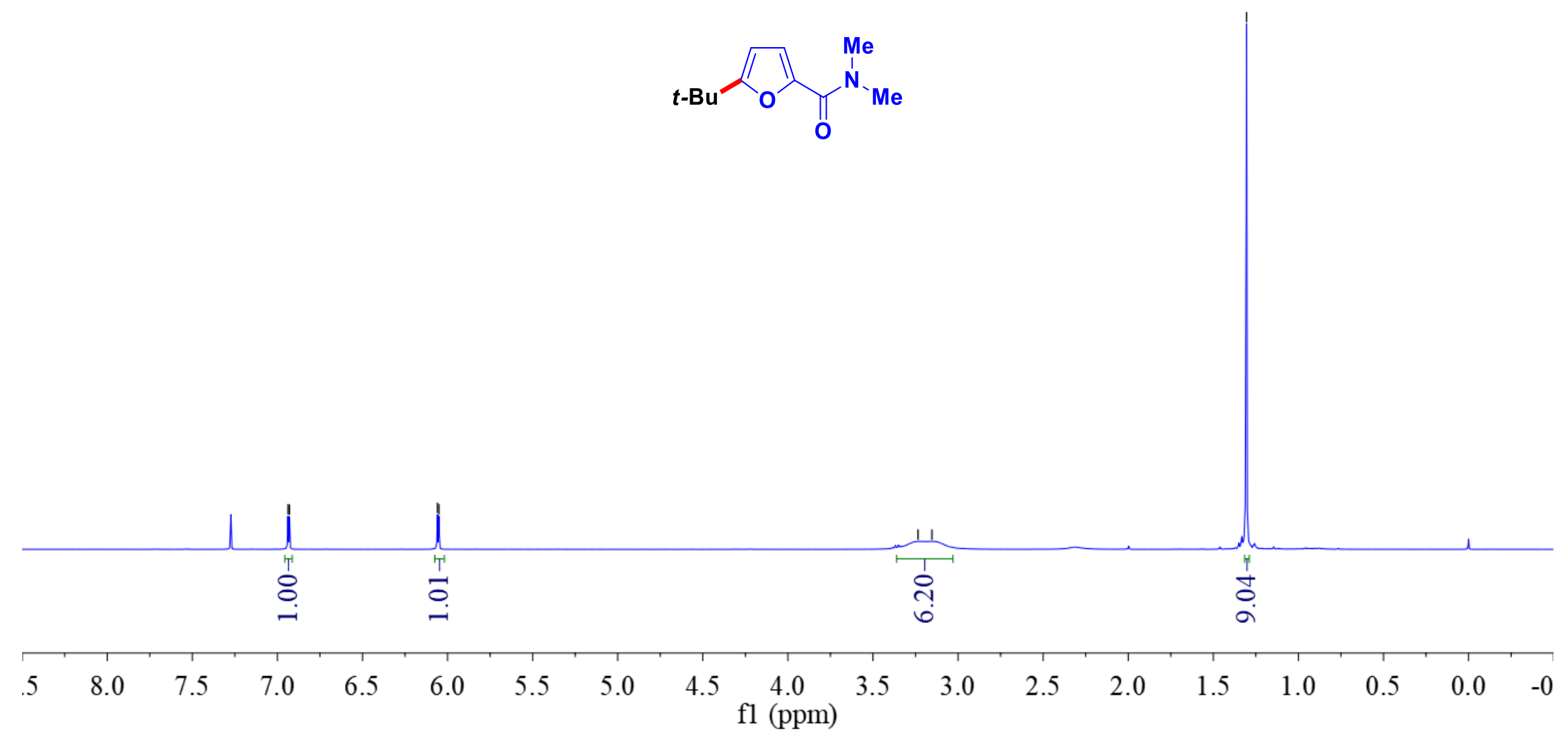


Figure S-29. ${ }^{13} \mathrm{C}\left\{{ }^{1} \mathrm{H}\right\}$ NMR spectrum of (1b) $\left(\mathrm{CDCl}_{3}, 100 \mathrm{MHz}\right)$ 5-(tert-butyl)- $N$, $N$-dimethylfuran-2-carboxamide

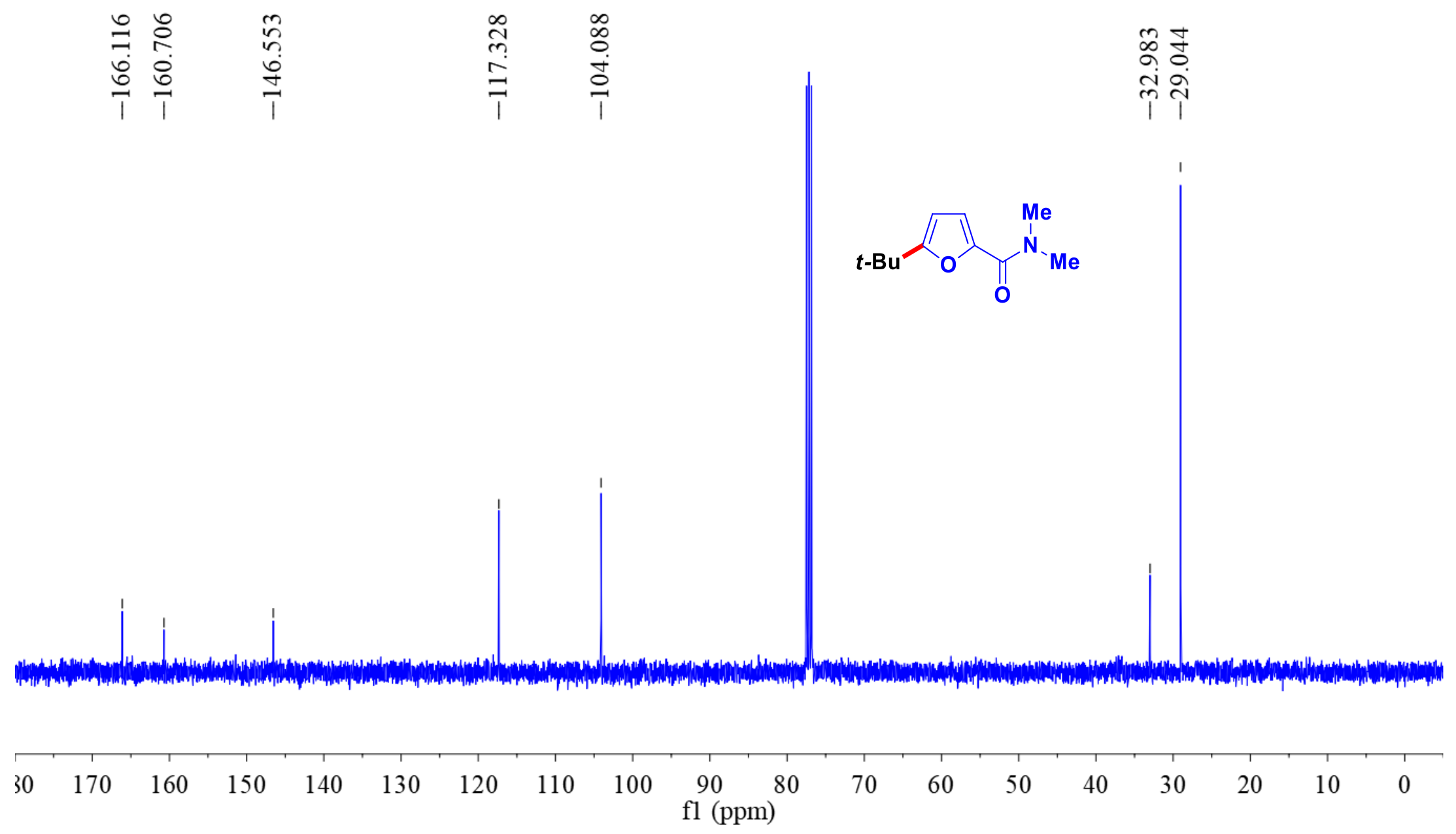


Figure S-30. ${ }^{1} \mathrm{H}$ NMR spectrum of (1c) $\left(\mathrm{CDCl}_{3}, 400 \mathrm{MHz}\right)$ 5-(tert-butyl)- $\boldsymbol{N}$, $\boldsymbol{N}$-diethylfuran-2-carboxamide
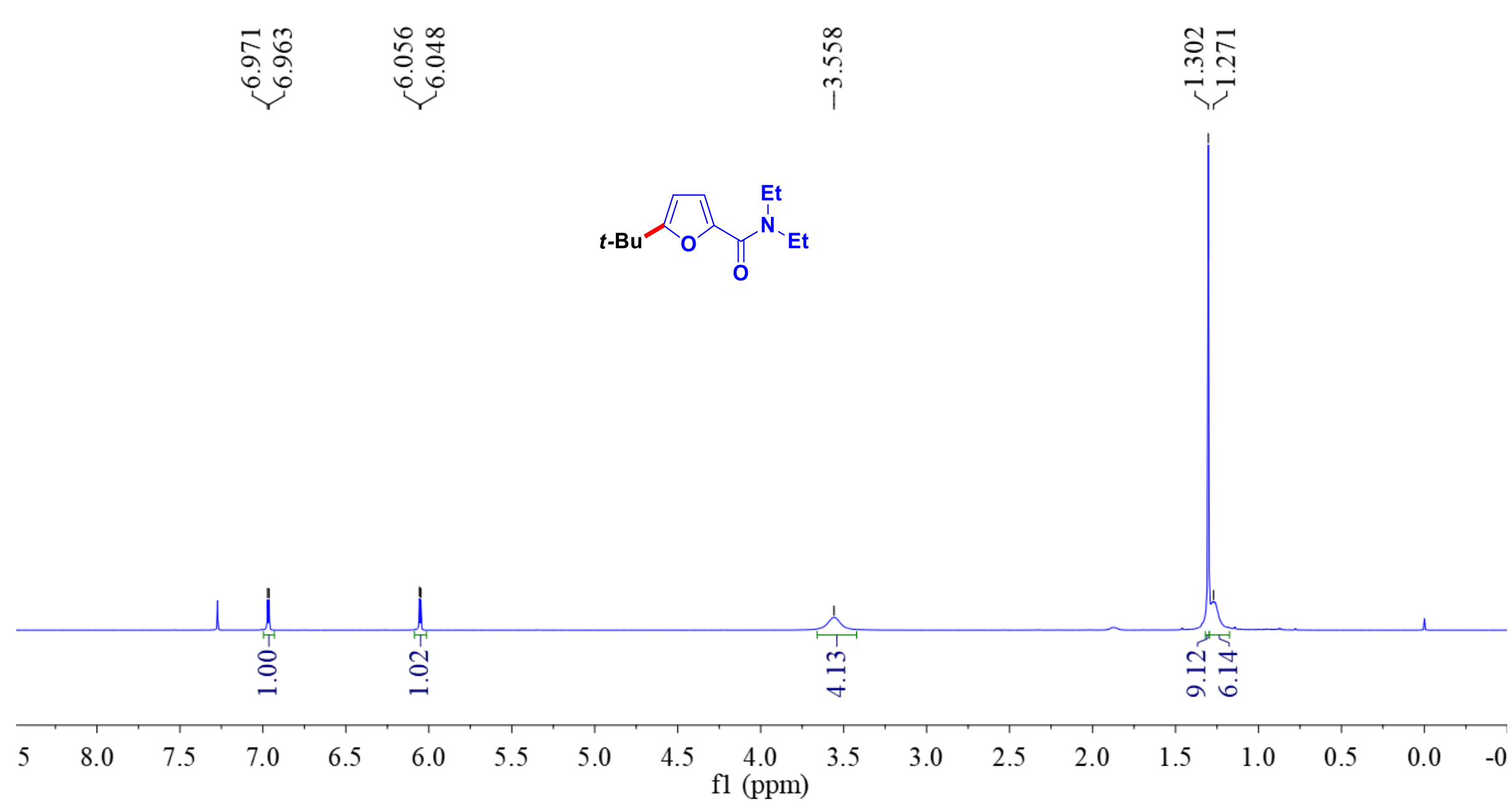

ำ 
Figure S-31. ${ }^{13} \mathrm{C}\left\{{ }^{1} \mathrm{H}\right\}$ NMR spectrum of (1c) $\left(\mathrm{CDCl}_{3}, 100 \mathrm{MHz}\right)$ 5-(tert-butyl)- $\boldsymbol{N}$, $\boldsymbol{N}$-diethylfuran-2-carboxamide

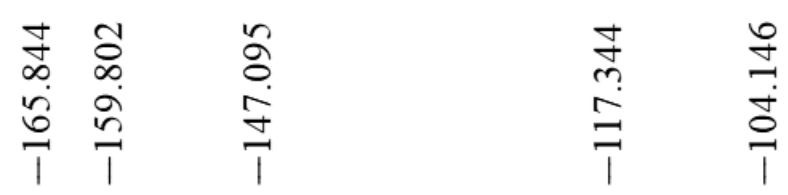$$
\text { হุ, }
$$$$
\text { iิ }
$$<smiles>CCN(CC)C(=O)c1ccc(C(C)(C)C)o1</smiles>

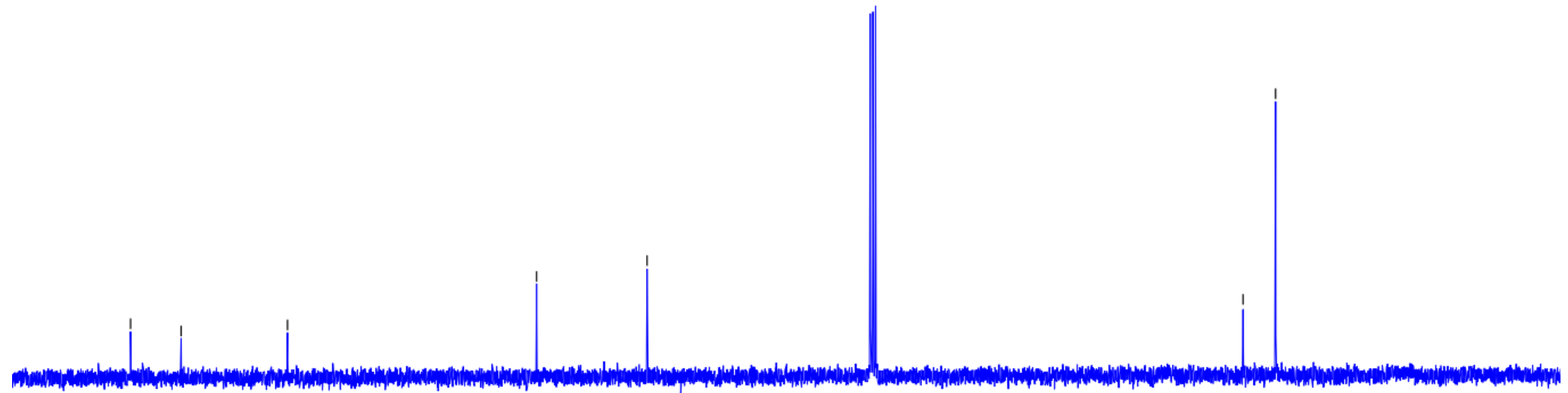

$\begin{array}{lllllll}30 & 170 & 160 & 150 & 140 & 130 & 120\end{array}$

$\begin{array}{lllllllll}90 \quad 80 & 70 & 60 & 50 & 40 & 30 & 20 & 10 & 0\end{array}$


Figure S-32. ${ }^{1} \mathrm{H}$ NMR spectrum of (1d) $\left(\mathrm{CDCl}_{3}, 400 \mathrm{MHz}\right) \mathrm{N}$-(2-bromophenyl)-5-(tert-butyl)- $\mathrm{N}$-methylfuran-2-carboxamide
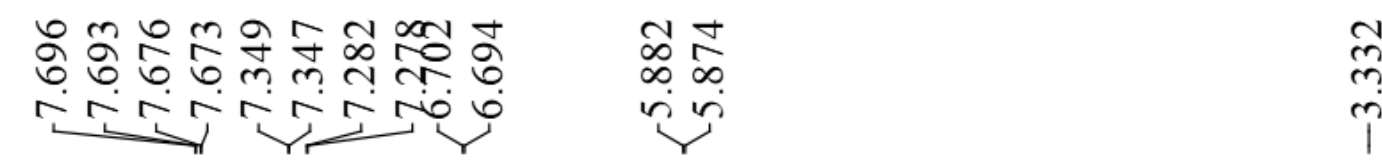

m
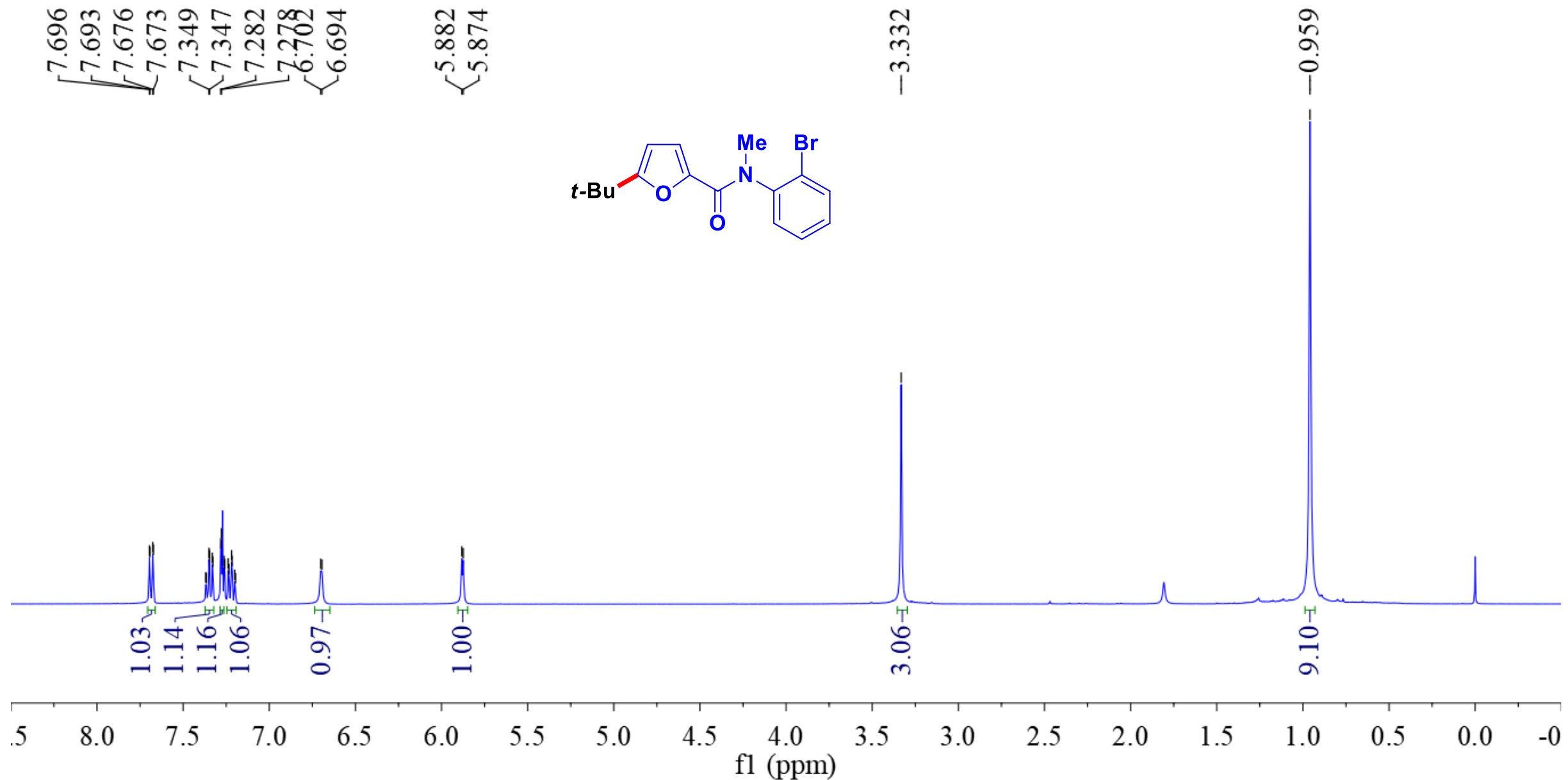
Figure S-33. ${ }^{13} \mathrm{C}\left\{{ }^{1} \mathrm{H}\right\}$ NMR spectrum of (1d) $\left(\mathrm{CDCl}_{3}, 100 \mathrm{MHz}\right) \mathrm{N}$-(2-bromophenyl)-5-(tert-butyl)- $N$-methylfuran-2-carboxamide

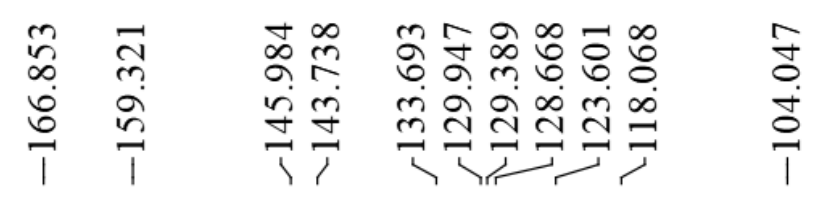

ণุ?

$\mathfrak{i} \stackrel{i}{i}$

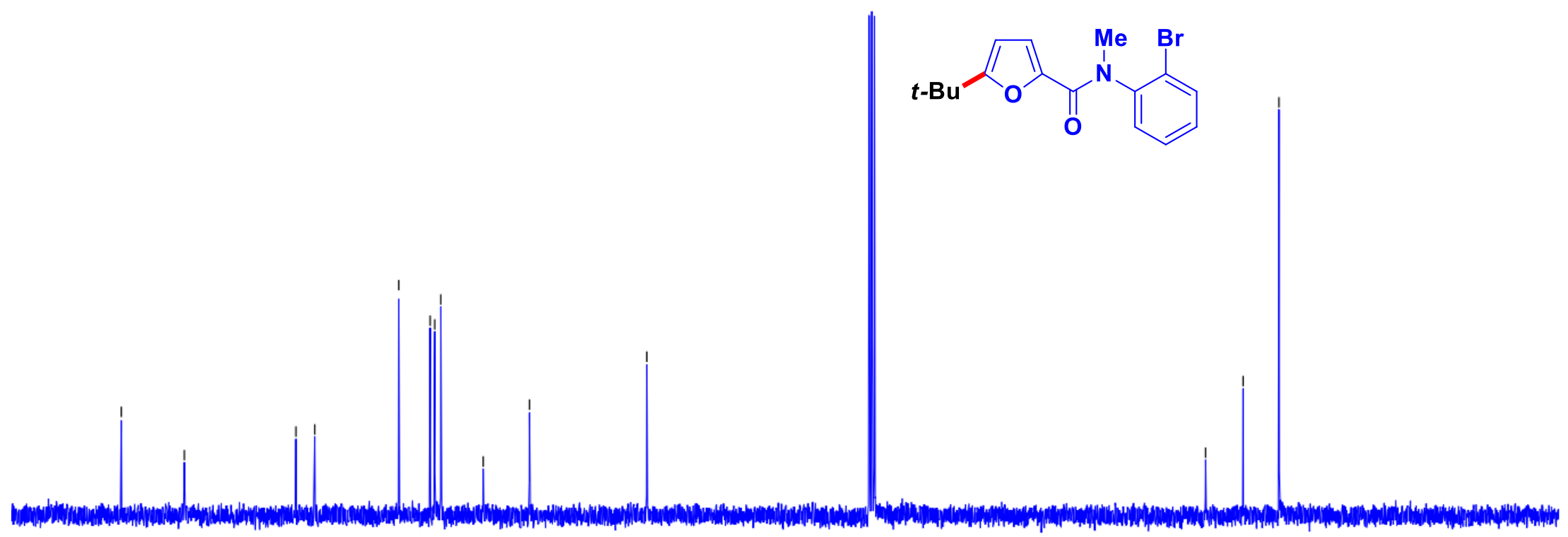


Figure S-34. ${ }^{1} \mathrm{H}$ NMR spectrum of (1e) $\left(\mathrm{CDCl}_{3}, 400 \mathrm{MHz}\right)$ 5-(tert-butyl)- $N$-(3-methoxyphenyl)- $N$-methylfuran-2-carboxamide

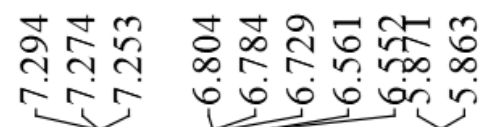

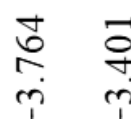

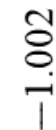
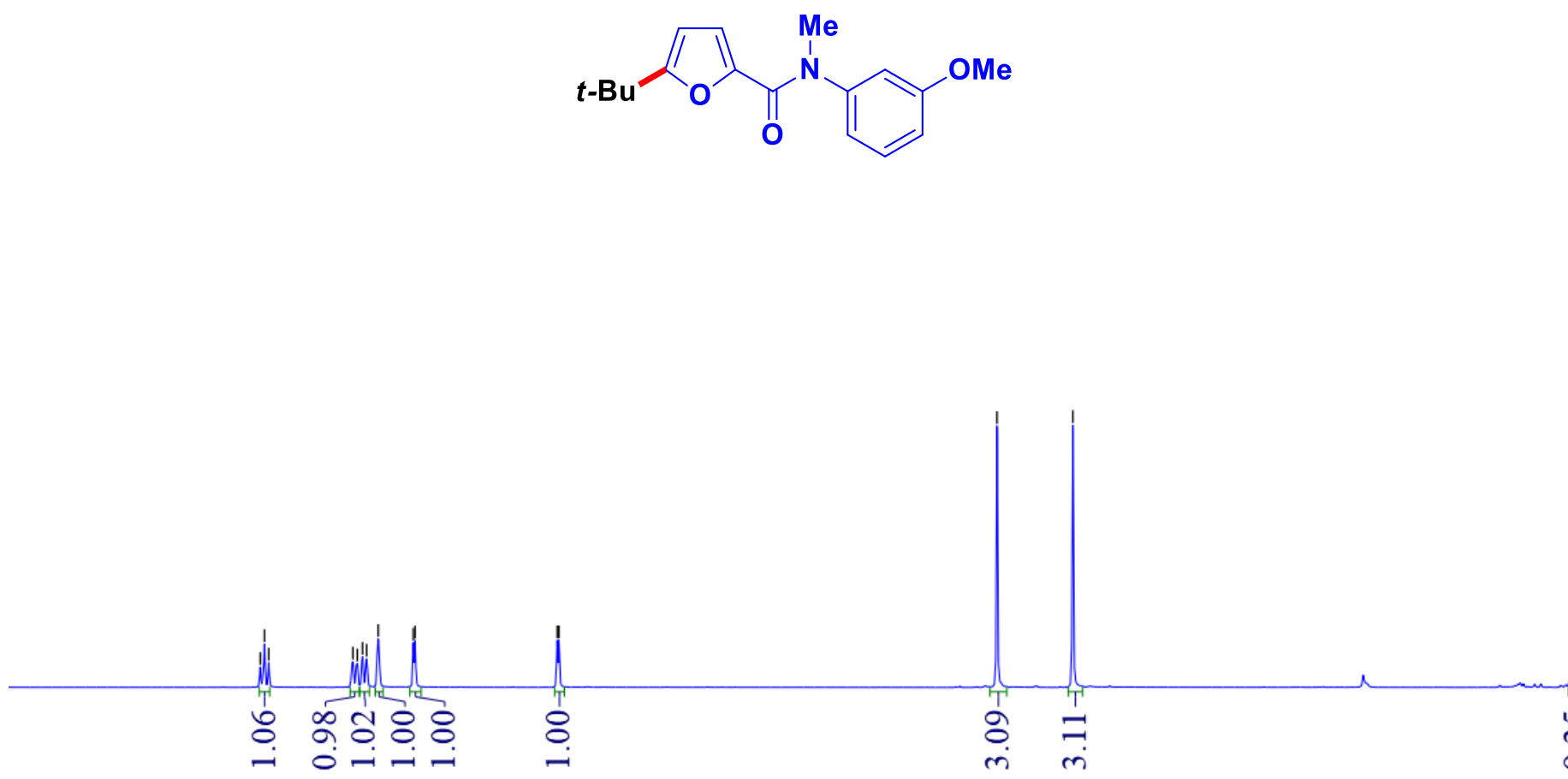

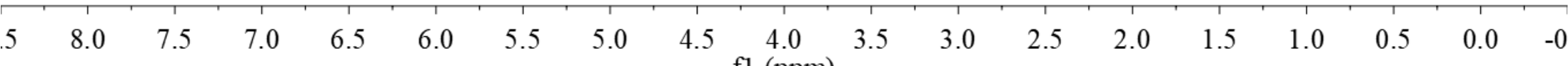


Figure S-35. ${ }^{13} \mathrm{C}\left\{{ }^{1} \mathrm{H}\right\}$ NMR spectrum of (1e) $\left(\mathrm{CDCl}_{3}, 100 \mathrm{MHz}\right)$ 5-(tert-butyl)- $N$-(3-methoxyphenyl)-N-methylfuran-2-carboxamide

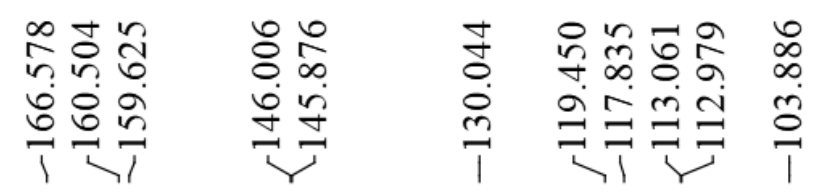
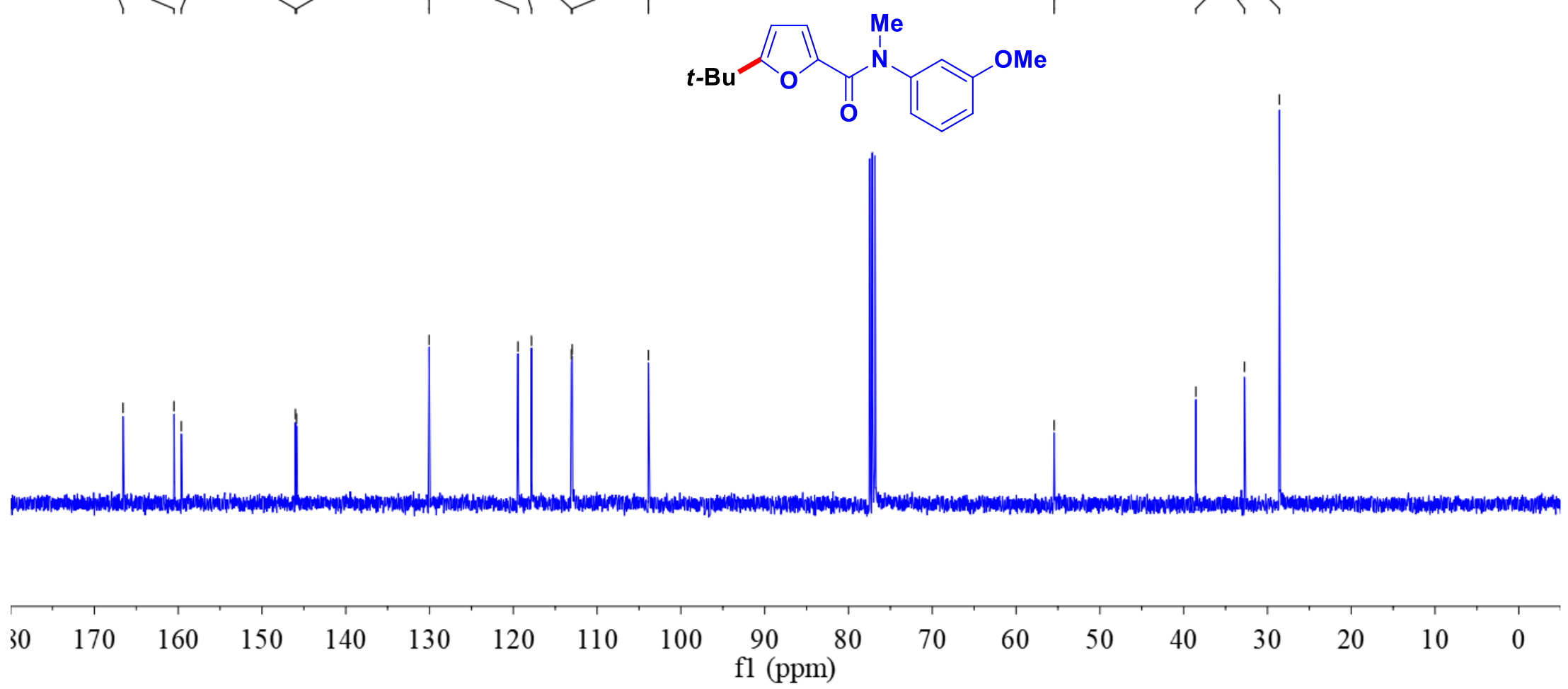
Figure S-36. ${ }^{1} \mathrm{H}$ NMR spectrum of (1f) $\left(\mathrm{CDCl}_{3}, 400 \mathrm{MHz}\right)(\mathbf{5}$-(tert-butyl)furan-2-yl)(pyrrolidin-1-yl)methanone
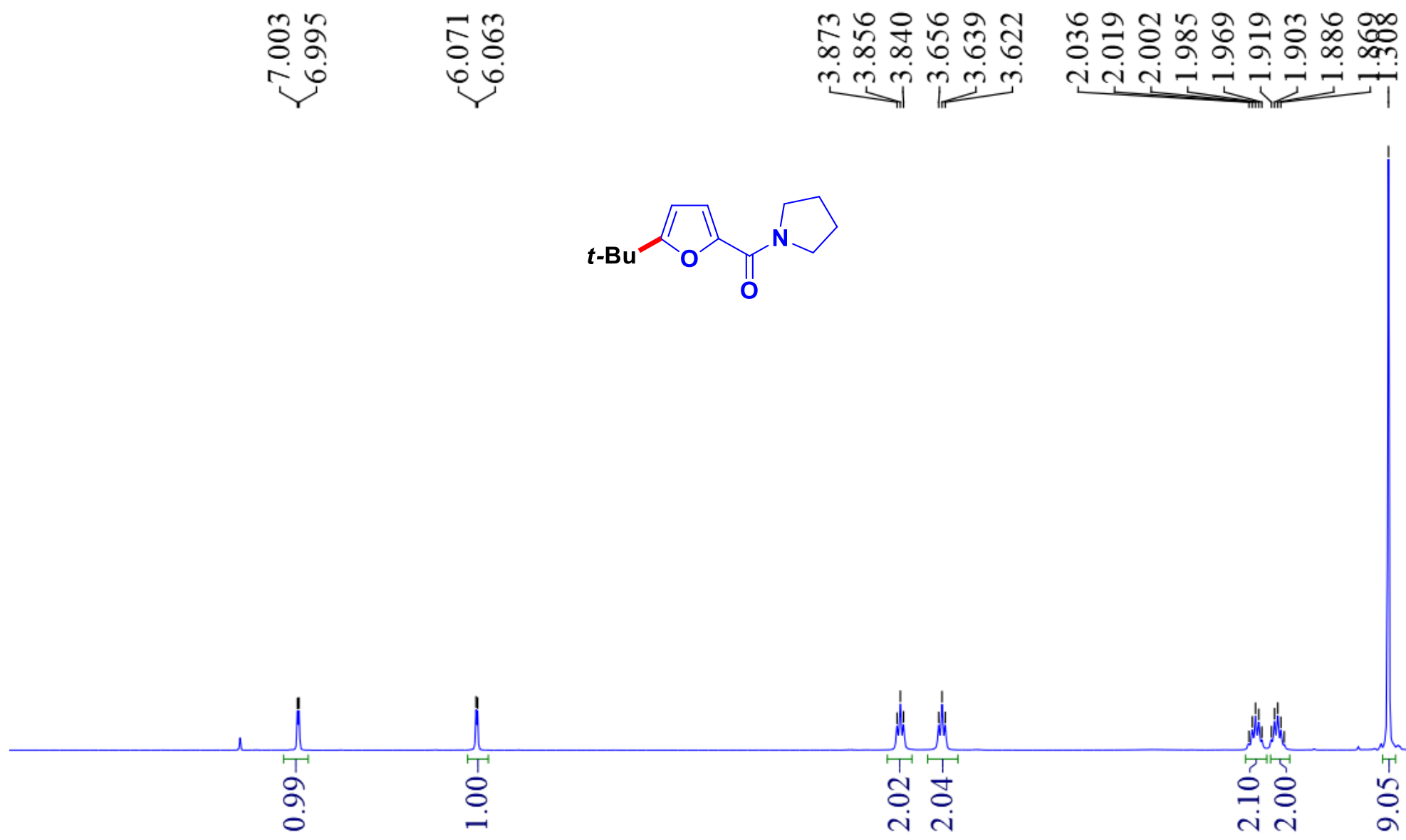

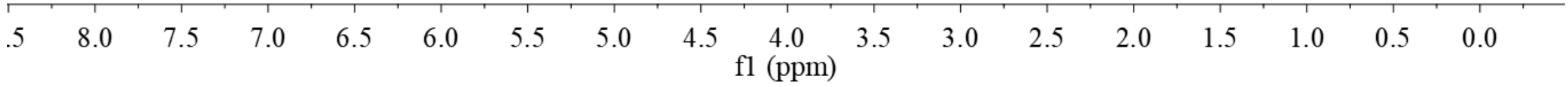


Figure S-37. ${ }^{13} \mathrm{C}\left\{{ }^{1} \mathrm{H}\right\}$ NMR spectrum of (1f) $\left(\mathrm{CDCl}_{3}, 100 \mathrm{MHz}\right)($ 5-(tert-butyl)furan-2-yl)(pyrrolidin-1-yl)methanone

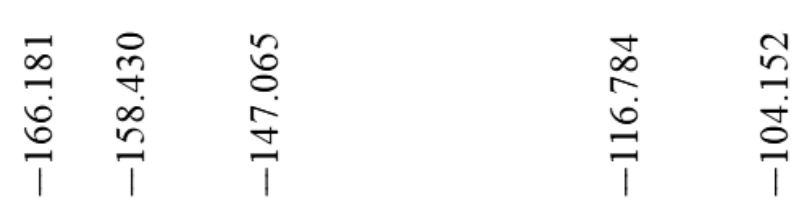

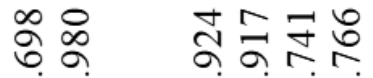$$
\text { Fं }
$$

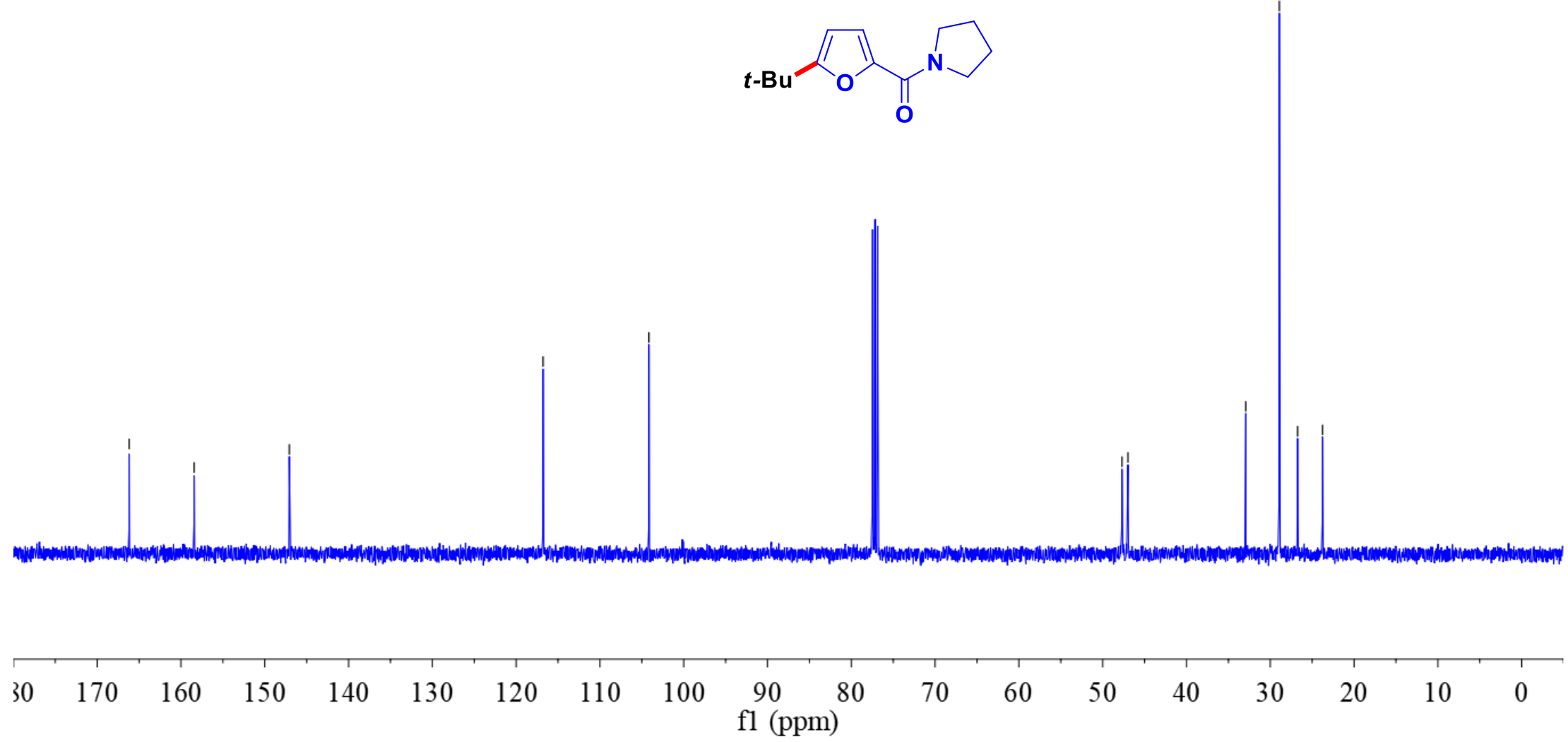


Figure S-38. ${ }^{1} \mathrm{H}$ NMR spectrum of (1g) $\left(\mathrm{CDCl}_{3}, 400 \mathrm{MHz}\right)(\mathbf{5}$-(tert-butyl)furan-2-yl)(piperidin-1-yl)methanone

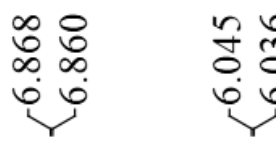
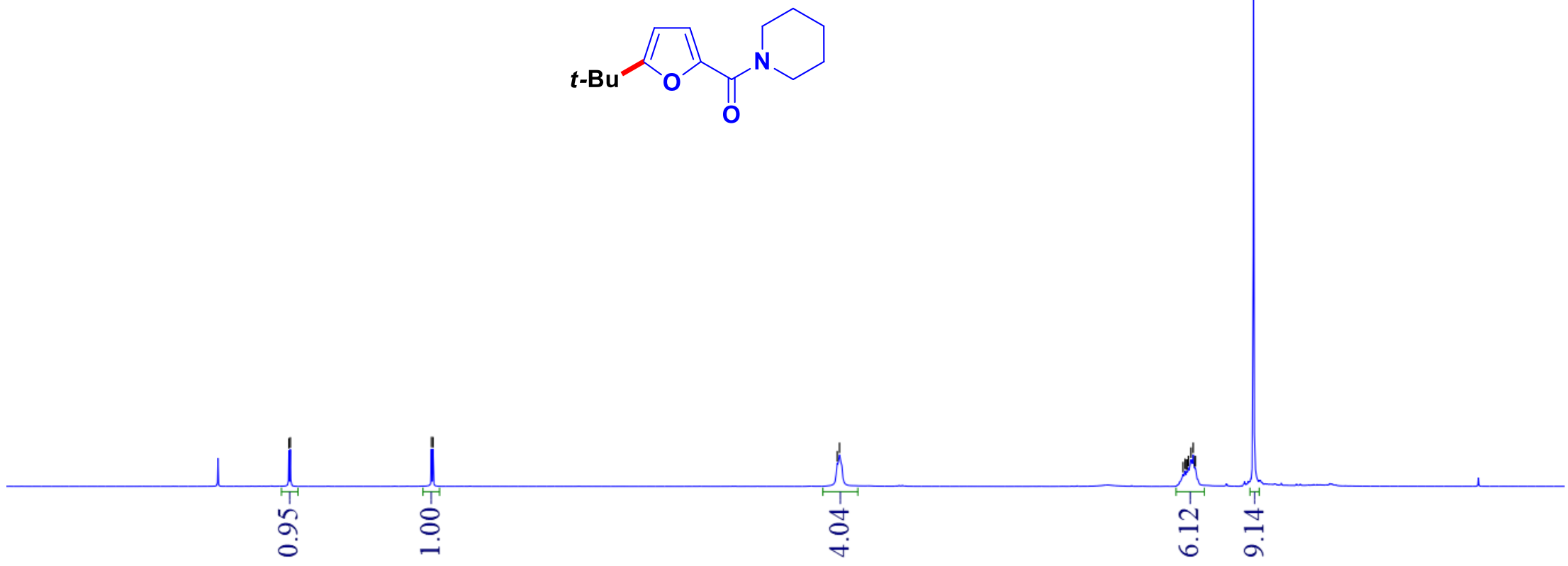

$5 \quad 8.0 \quad 7.5 \quad 7.0$

$\begin{array}{lllll}6.5 & 6.0 & 5.5 & 5.0 & 4.5\end{array} \quad 4$.

4.0

3.5

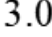

2.5 
Figure S-39. ${ }^{13} \mathrm{C}\left\{{ }^{1} \mathrm{H}\right\}$ NMR spectrum of $(\mathbf{1 g})\left(\mathrm{CDCl}_{3}, 100 \mathrm{MHz}\right)(5$-(tert-butyl)furan-2-yl)(piperidin-1-yl)methanone

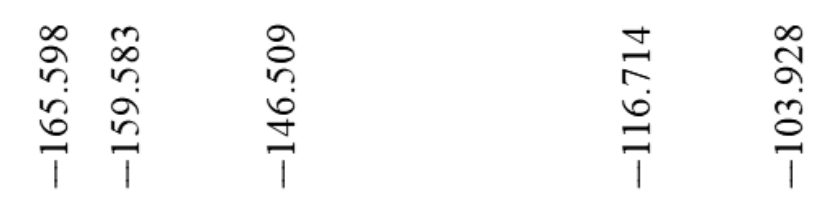

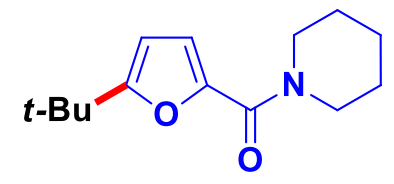

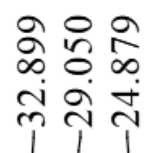

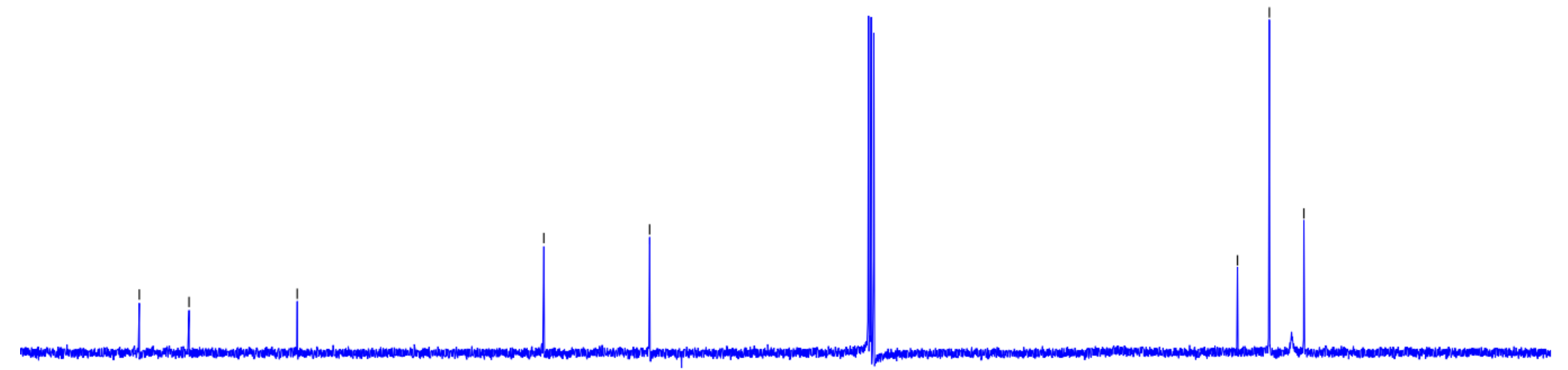

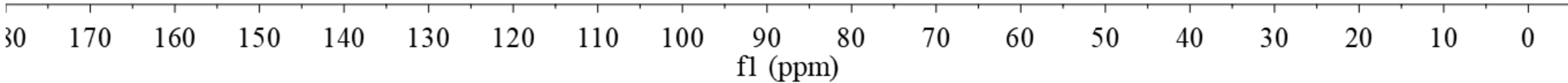


Figure S-40. ${ }^{1} \mathrm{H}$ NMR spectrum of (1h) $\left(\mathrm{CDCl}_{3}, 400 \mathrm{MHz}\right)$ 5-(tert-butyl)- $N$-methoxy- $N$-methylfuran-2-carboxamide

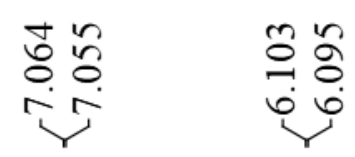

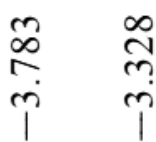

m
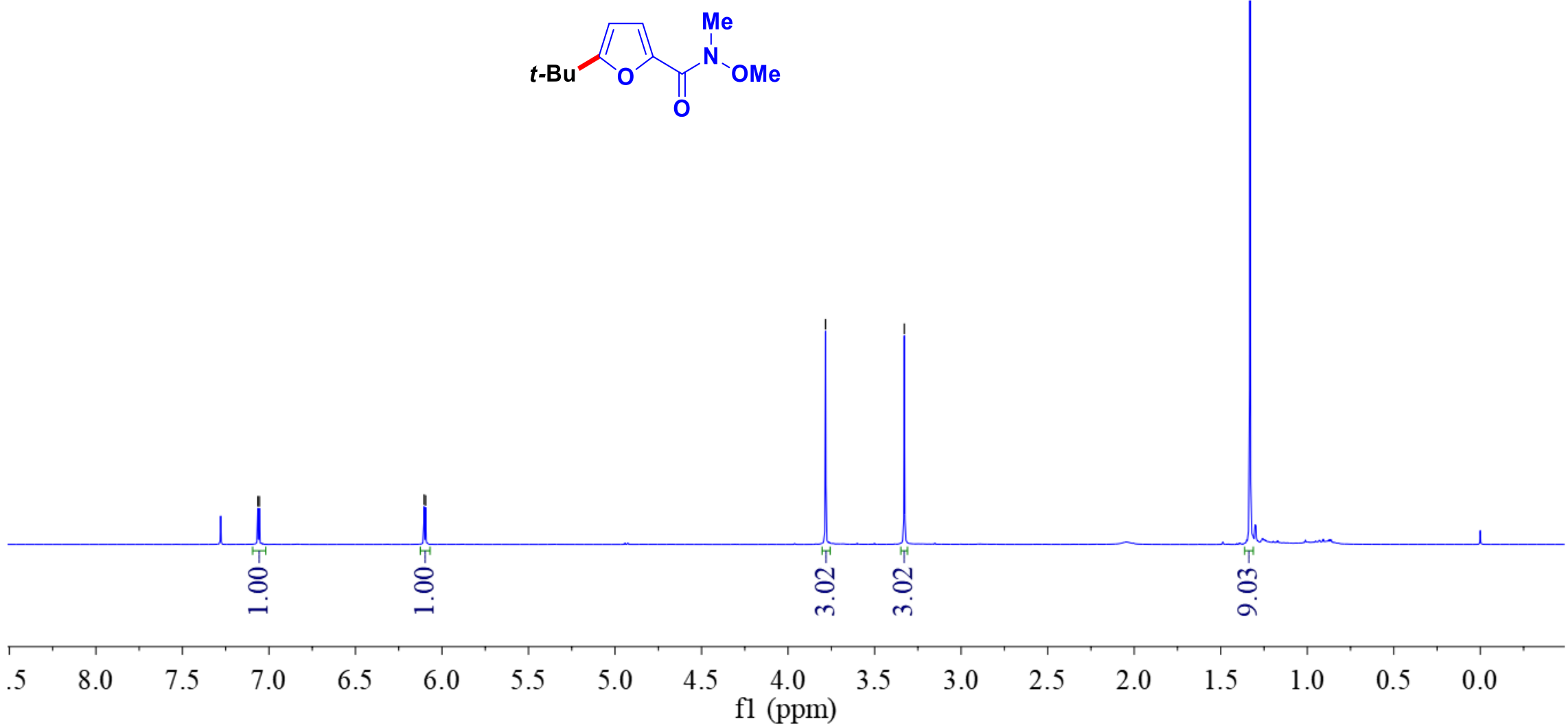
Figure S-41. ${ }^{13} \mathrm{C}\left\{{ }^{1} \mathrm{H}\right\}$ NMR spectrum of (1h) $\left(\mathrm{CDCl}_{3}, 100 \mathrm{MHz}\right)$ 5-(tert-butyl)- $N$-methoxy- $N$-methylfuran-2-carboxamide
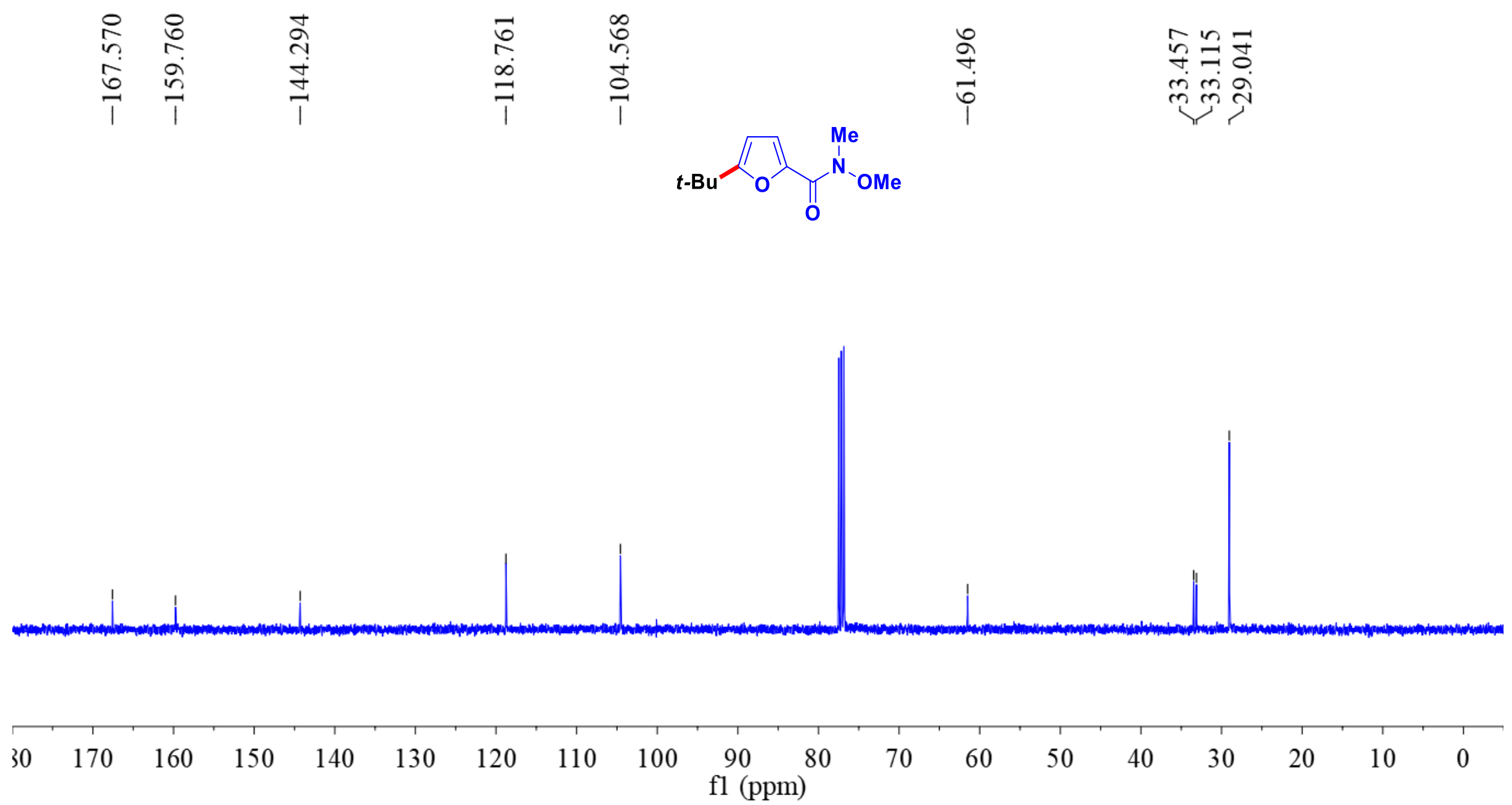
Figure S-42. ${ }^{1} \mathrm{H}$ NMR spectrum of (1i) $\left(\mathrm{CDCl}_{3}, 400 \mathrm{MHz}\right)$ methyl 5-(tert-butyl)furan-2-carboxylate

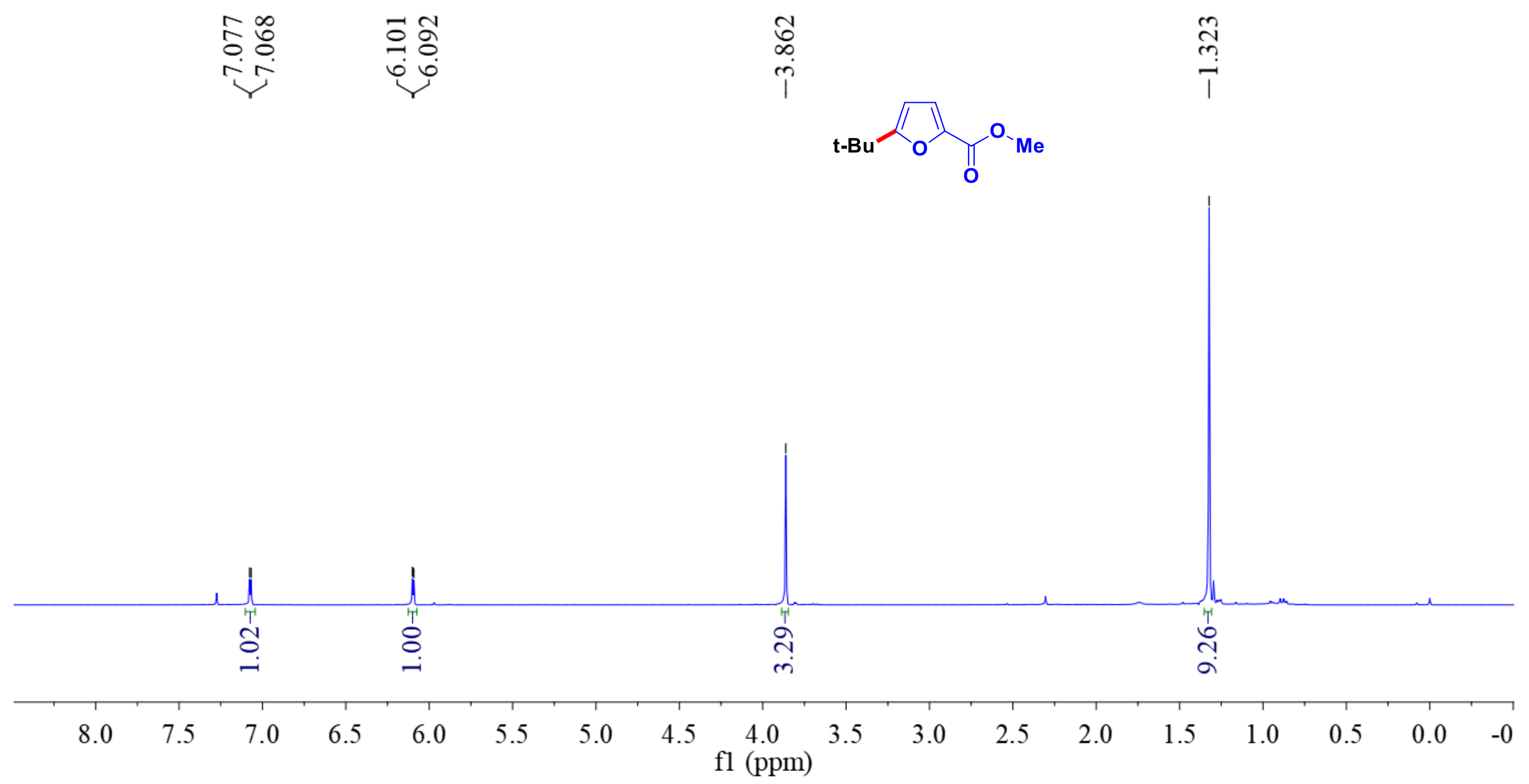


Figure S-43. ${ }^{13} \mathrm{C}\left\{{ }^{1} \mathrm{H}\right\}$ NMR spectrum of (1i) $\left(\mathrm{CDCl}_{3}, 100 \mathrm{MHz}\right)$ methyl 5-(tert-butyl)furan-2-carboxylate

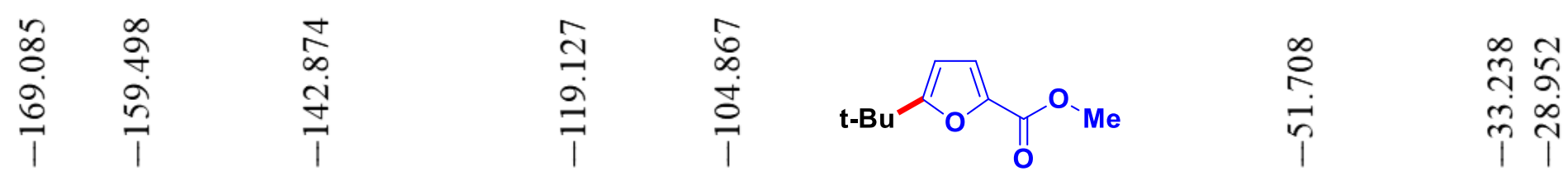

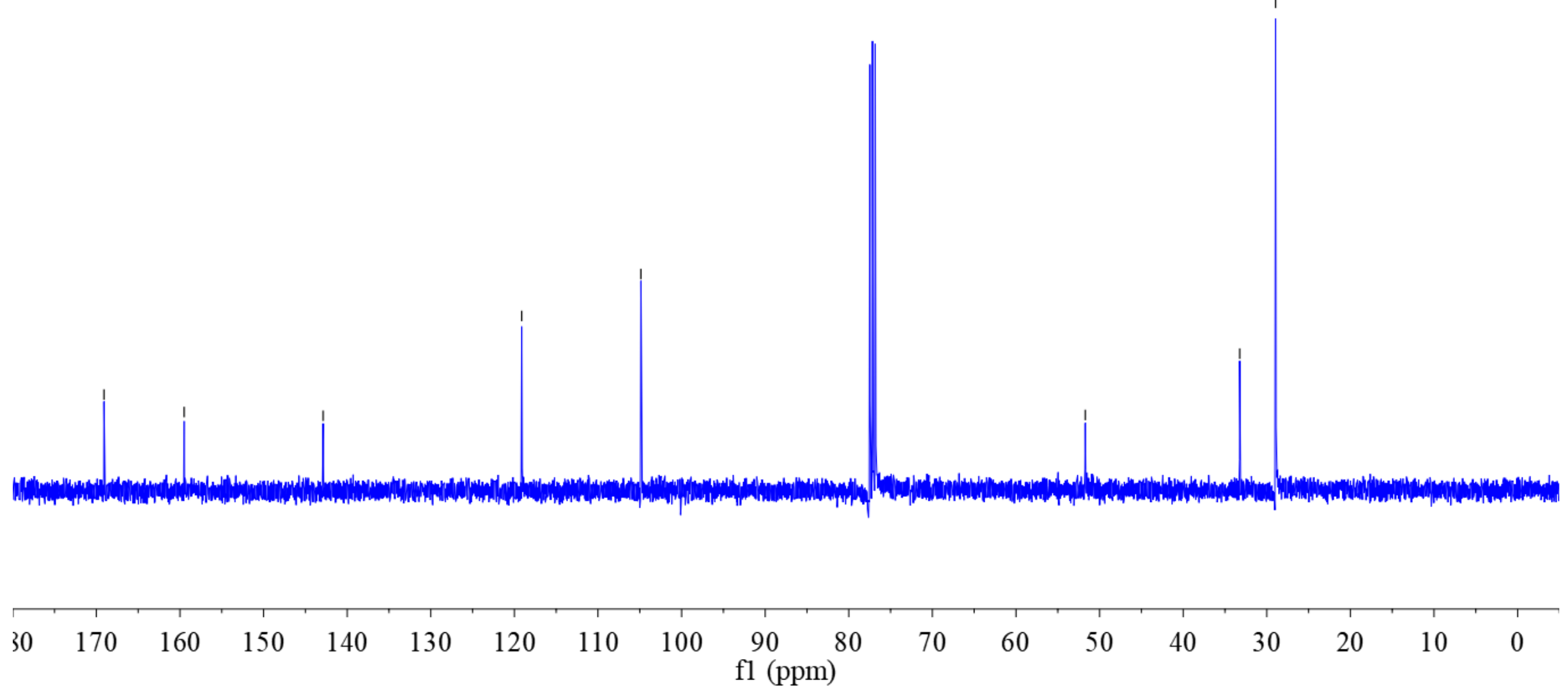


Figure S-44. ${ }^{1} \mathrm{H}$ NMR spectrum of $(\mathbf{1 j})\left(\mathrm{CDCl}_{3}, 400 \mathrm{MHz}\right)$ ethyl 5-(tert-butyl)furan-2-carboxylate
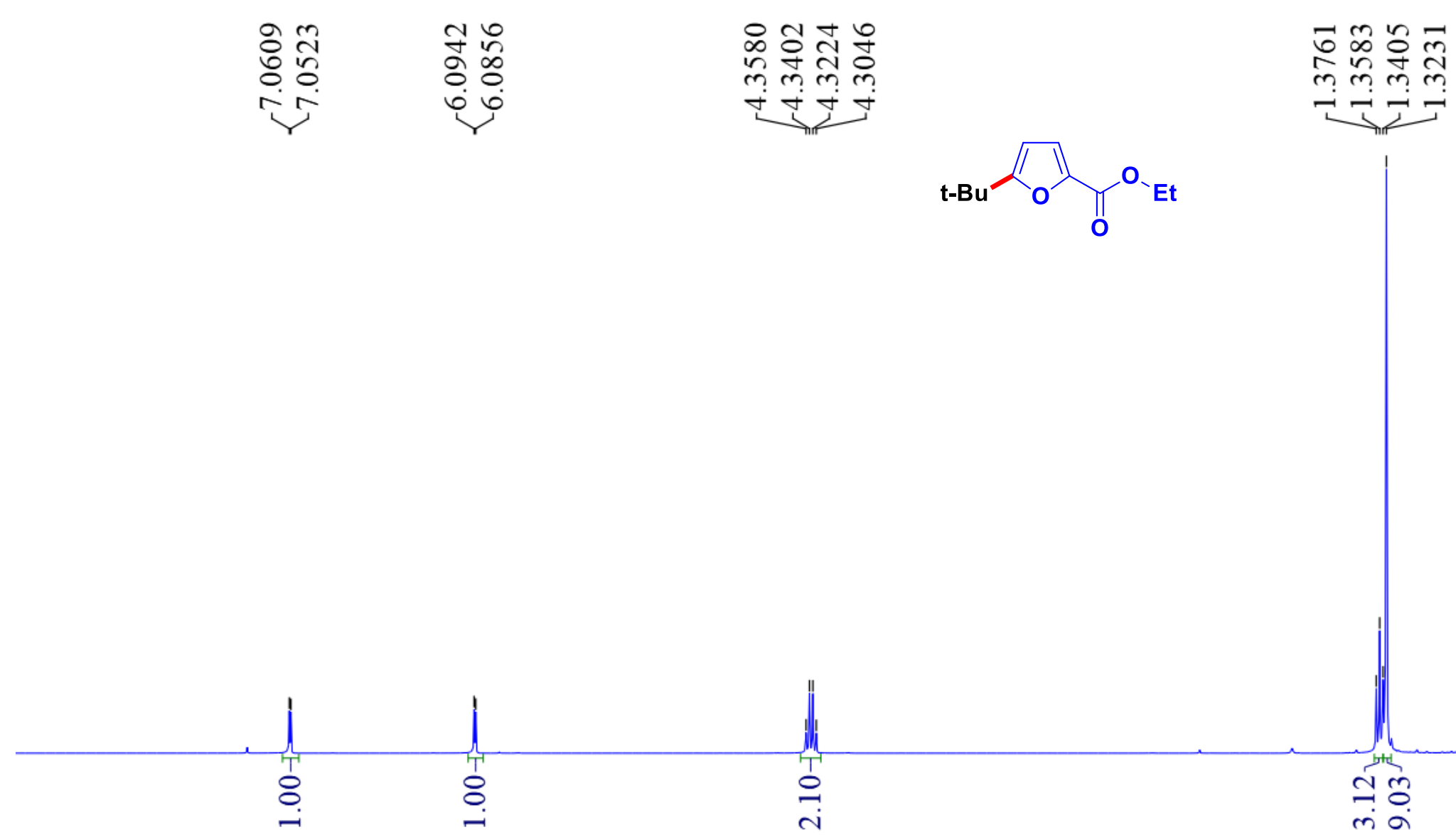

\begin{tabular}{|c|c|c|c|c|c|c|c|c|c|c|c|c|c|c|c|c|c|}
\hline 8.0 & 7.5 & 7.0 & 6.5 & 6.0 & 5.5 & 5.0 & 4.5 & $\begin{array}{c}4.0 \\
\text { fl }(\mathrm{ppm})\end{array}$ & 3.5 & 3.0 & 2.5 & 2.0 & 1.5 & 1.0 & 0.5 & 0.0 & -0 \\
\hline
\end{tabular}


Figure $\mathbf{S}-45 .{ }^{13} \mathrm{C}\left\{{ }^{1} \mathrm{H}\right\}$ NMR spectrum of $(\mathbf{1 j})\left(\mathrm{CDCl}_{3}, 100 \mathrm{MHz}\right)$ ethyl 5-(tert-butyl)furan-2-carboxylate

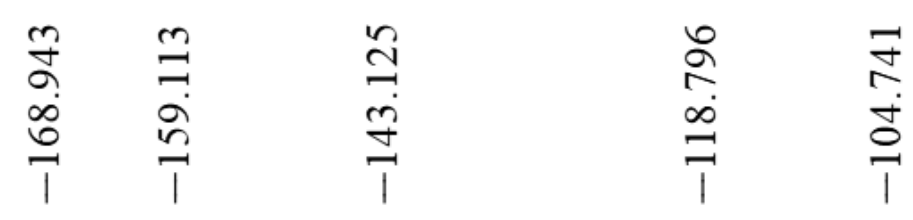

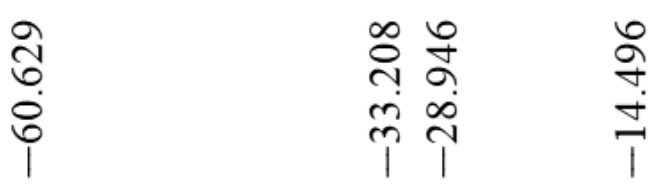
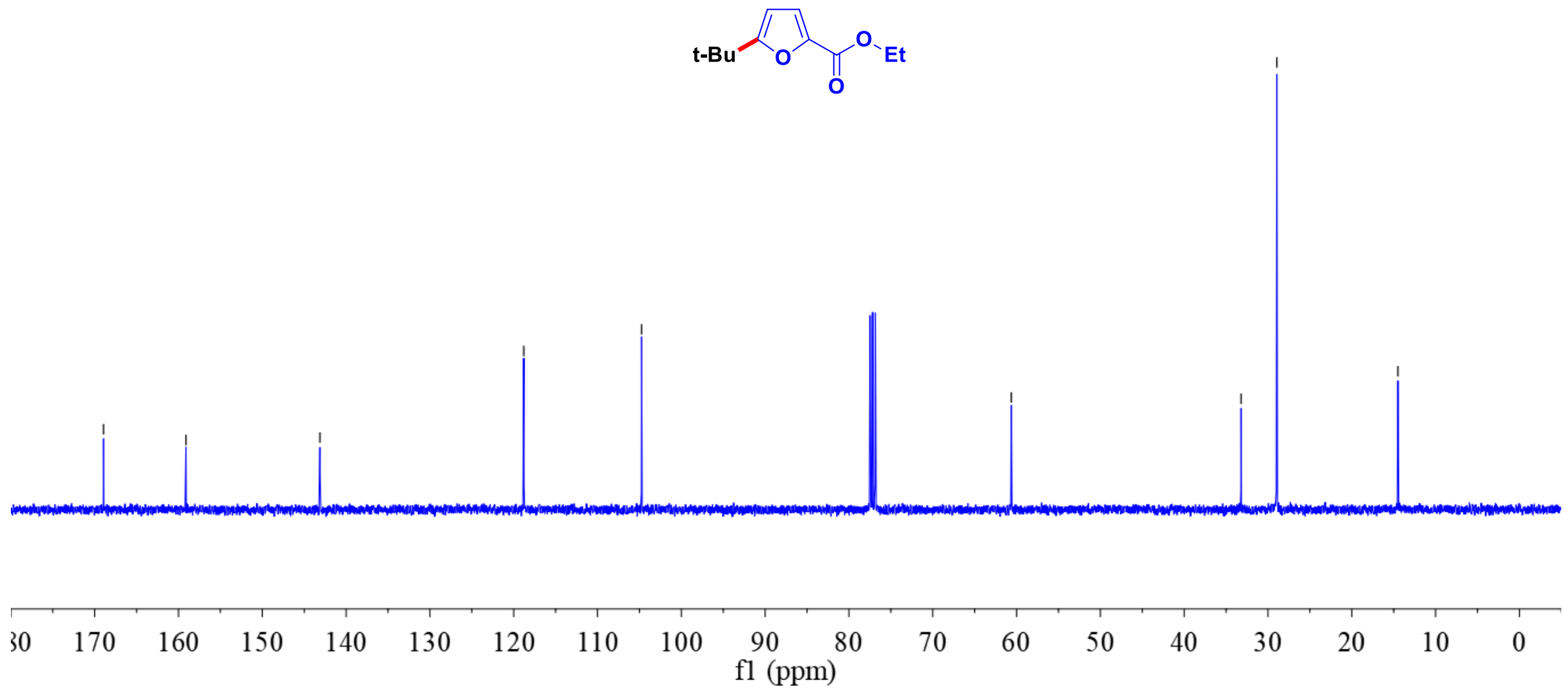
Figure S-46. ${ }^{1} \mathrm{H}$ NMR spectrum of (1k) $\left(\mathrm{CDCl}_{3}, 400 \mathrm{MHz}\right)$ isopropyl 5-(tert-butyl)furan-2-carboxylate

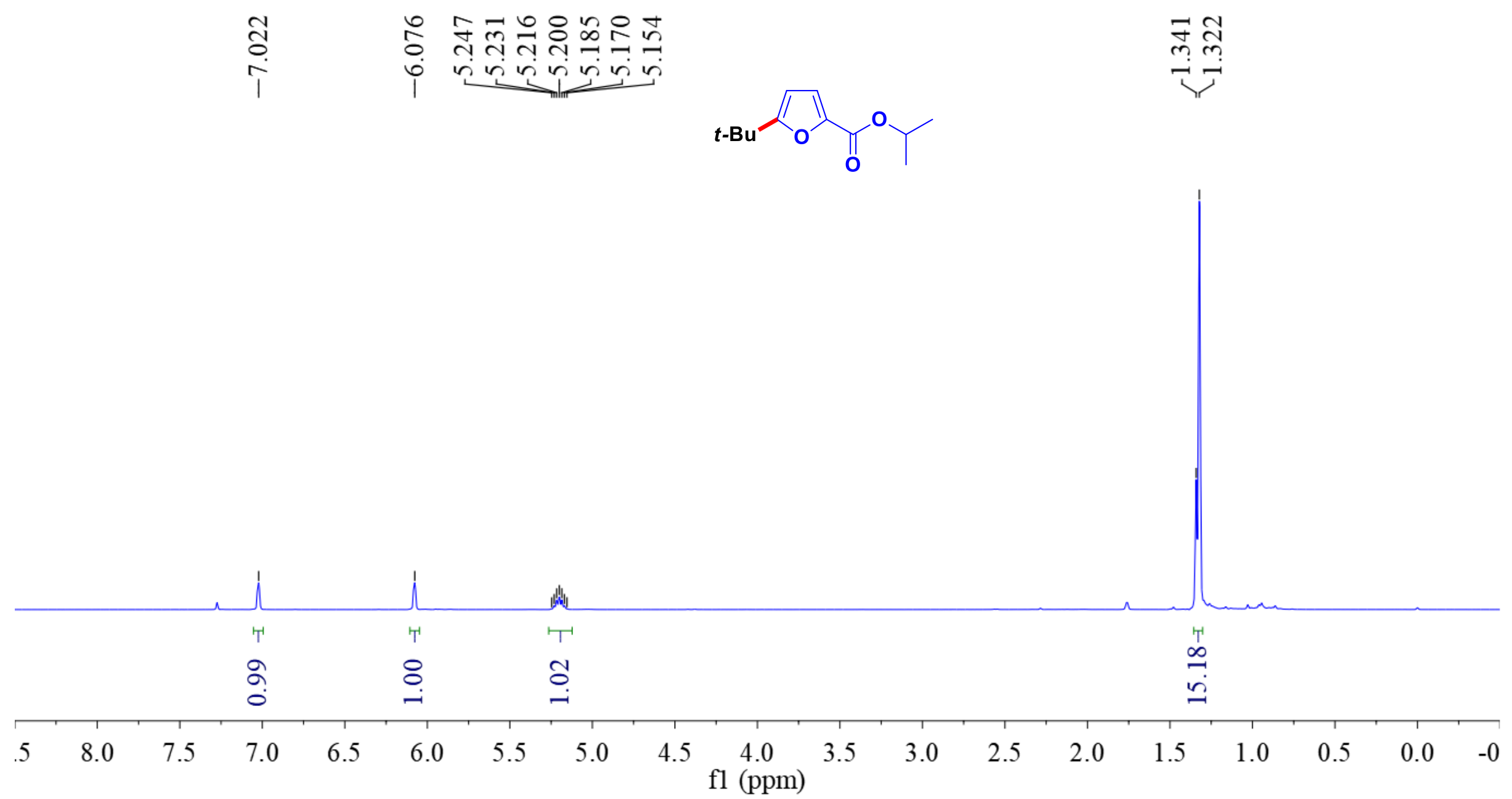


Figure $\mathbf{S - 4 7 .}{ }^{13} \mathrm{C}\left\{{ }^{1} \mathrm{H}\right\}$ NMR spectrum of (1k) $\left(\mathrm{CDCl}_{3}, 100 \mathrm{MHz}\right)$ isopropyl 5-(tert-butyl)furan-2-carboxylate

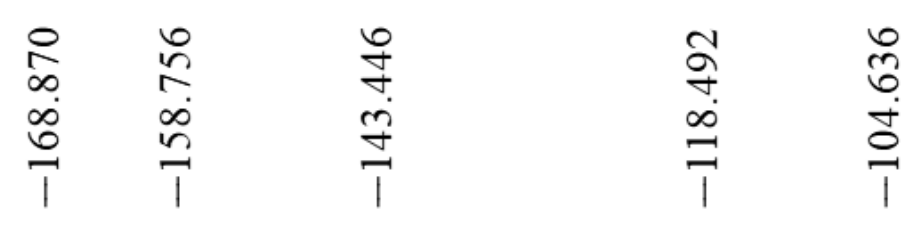
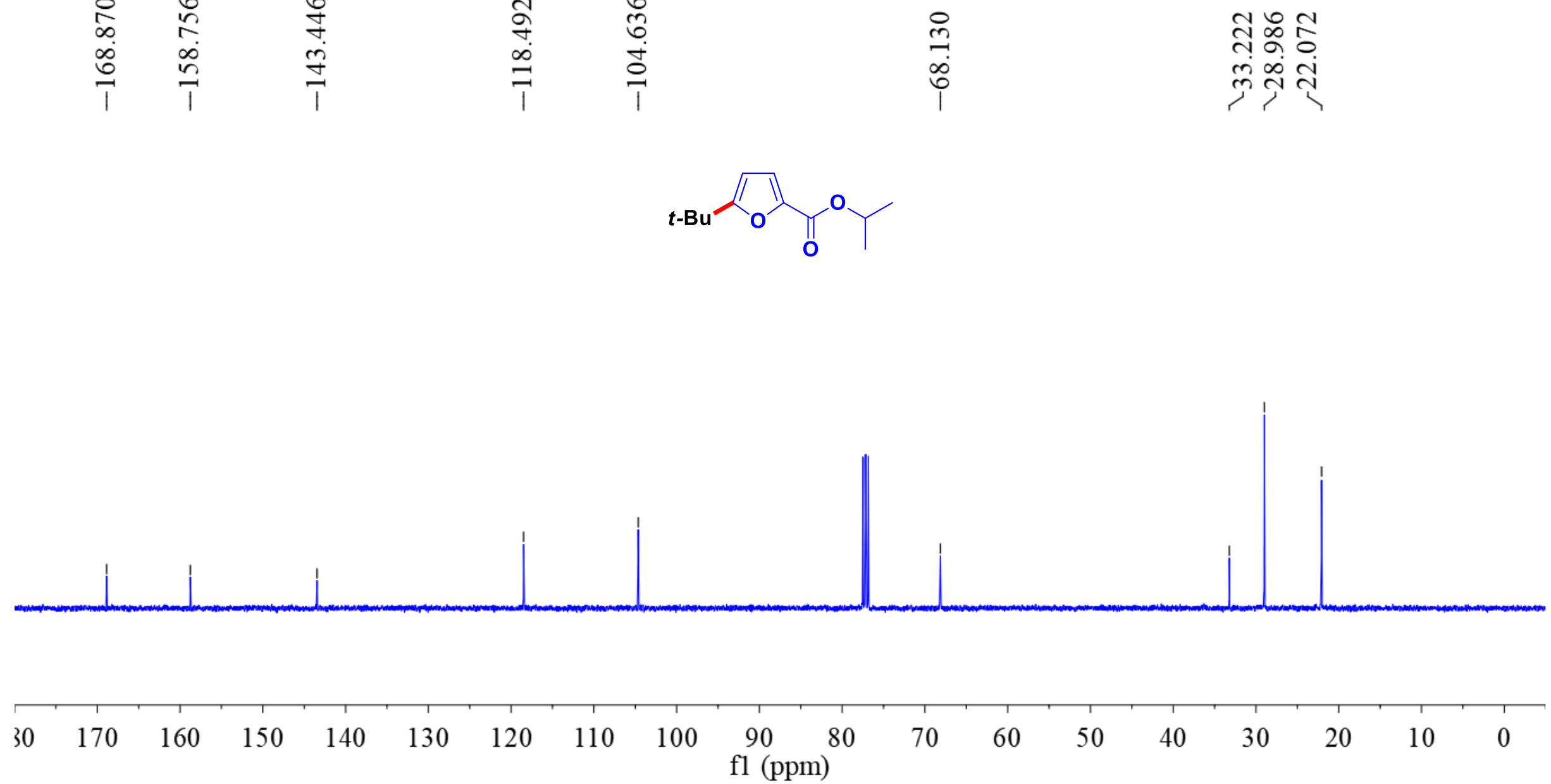
Figure S-48. ${ }^{1} \mathrm{H}$ NMR spectrum of (11) $\left(\mathrm{CDCl}_{3}, 400 \mathrm{MHz}\right)(\mathbf{5}$-(tert-butyl)furan-2-yl)(phenyl)methanone
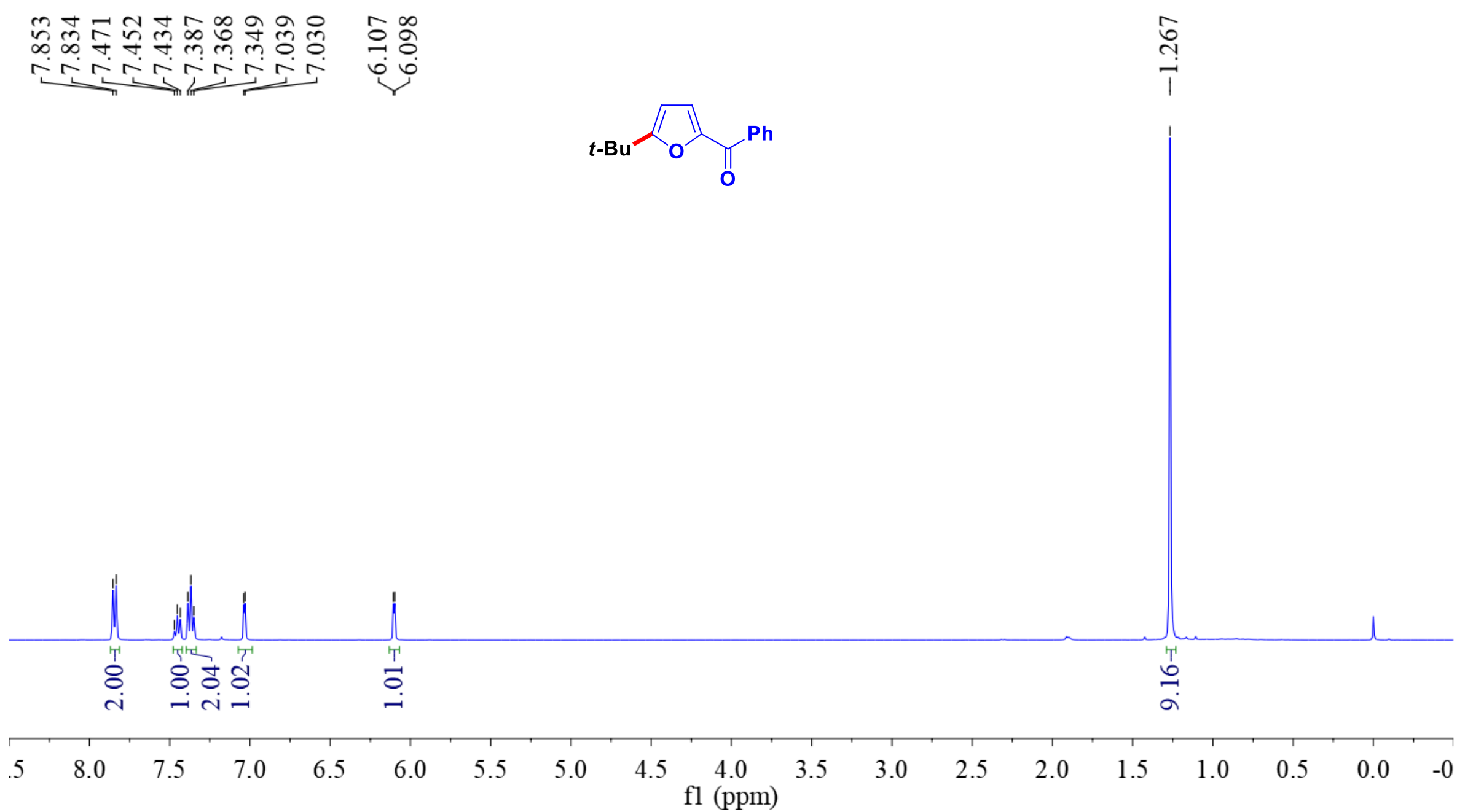
Figure S-49. ${ }^{13} \mathrm{C}\left\{{ }^{1} \mathrm{H}\right\}$ NMR spectrum of (11) $\left(\mathrm{CDCl}_{3}, 100 \mathrm{MHz}\right)($ 5-(tert-butyl)furan-2-yl)(phenyl)methanone

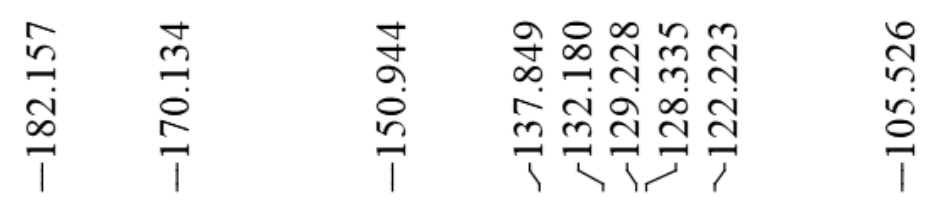

ㅎํ응

$\stackrel{i}{i}$
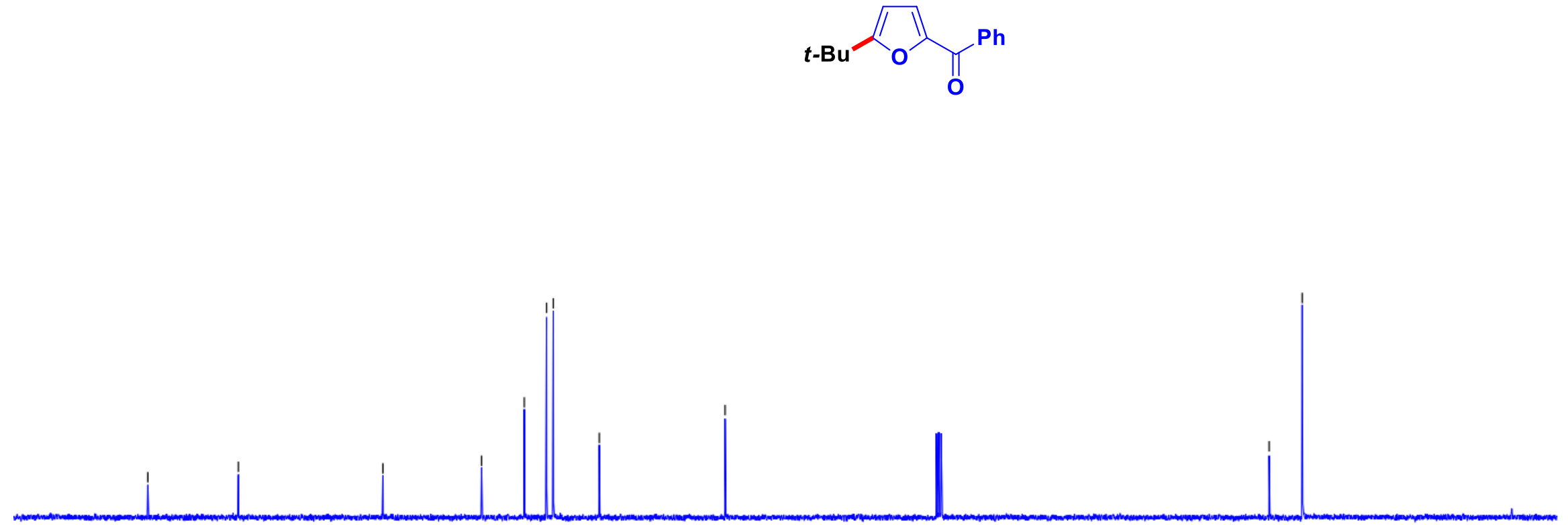

$\begin{array}{llllll}190 & 180 & 170 & 160 & 150-140 & 130\end{array}$

110100

10090

$80 \quad 70$

6050


Figure S-50. ${ }^{1} \mathrm{H}$ NMR spectrum of $(\mathbf{1 m})\left(\mathrm{CDCl}_{3}, 400 \mathrm{MHz}\right)$ 5-(tert-butyl)furan-2-carbaldehyde

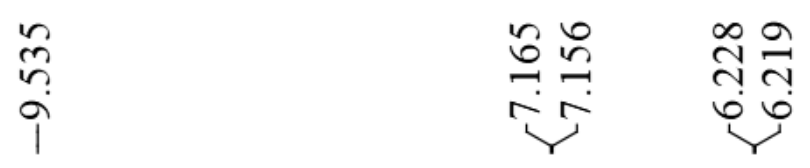

$\stackrel{n}{\frac{n}{3}}$
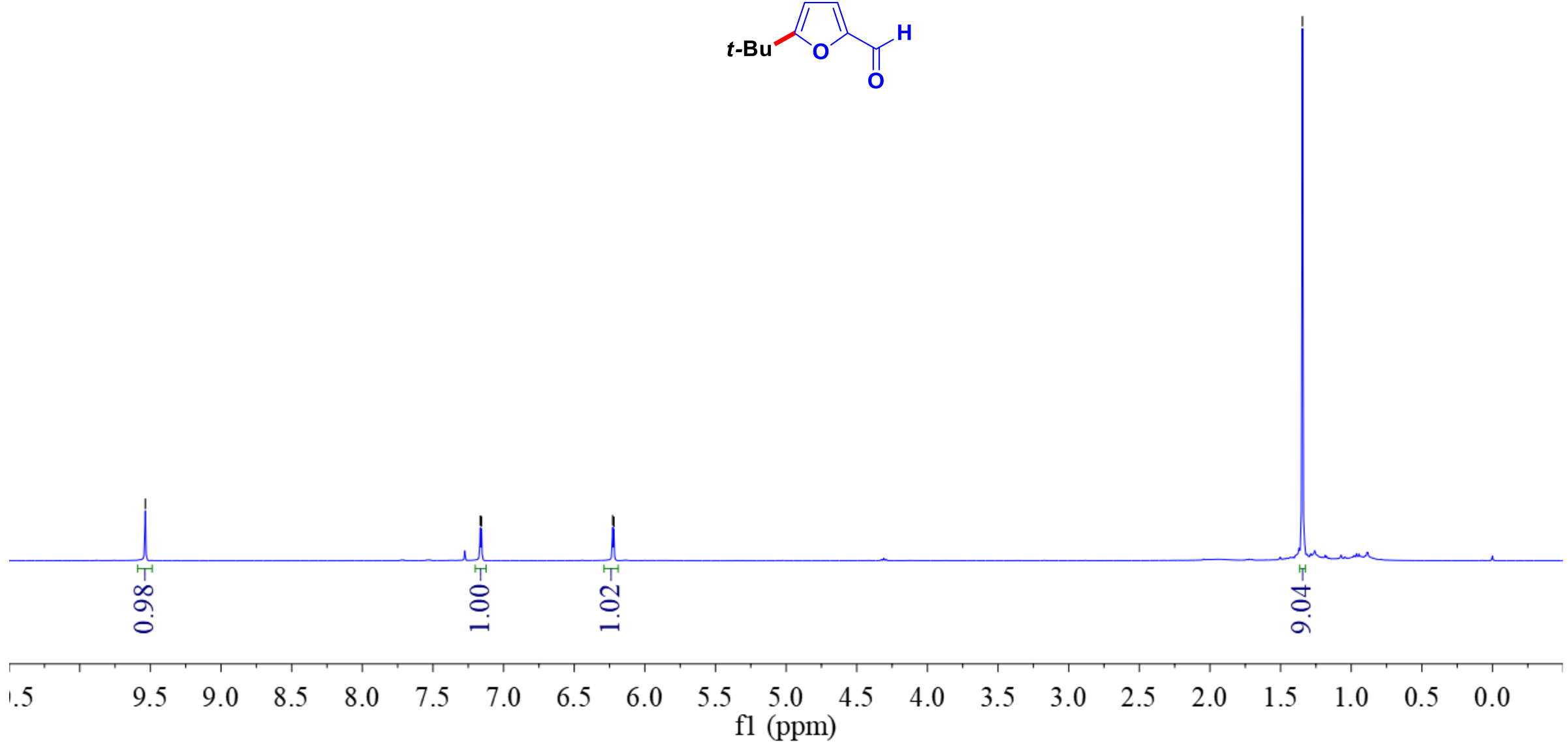
Figure S-51. ${ }^{13} \mathrm{C}\left\{{ }^{1} \mathrm{H}\right\}$ NMR spectrum of (1m) $\left(\mathrm{CDCl}_{3}, 100 \mathrm{MHz}\right)$ 5-(tert-butyl)furan-2-carbaldehyde

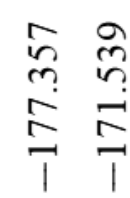
$m$
$\infty$

$n$
0
0
0 $\stackrel{\substack{* \\ \infty}}{n}$ $\ddot{m} \stackrel{\infty}{\sim}$
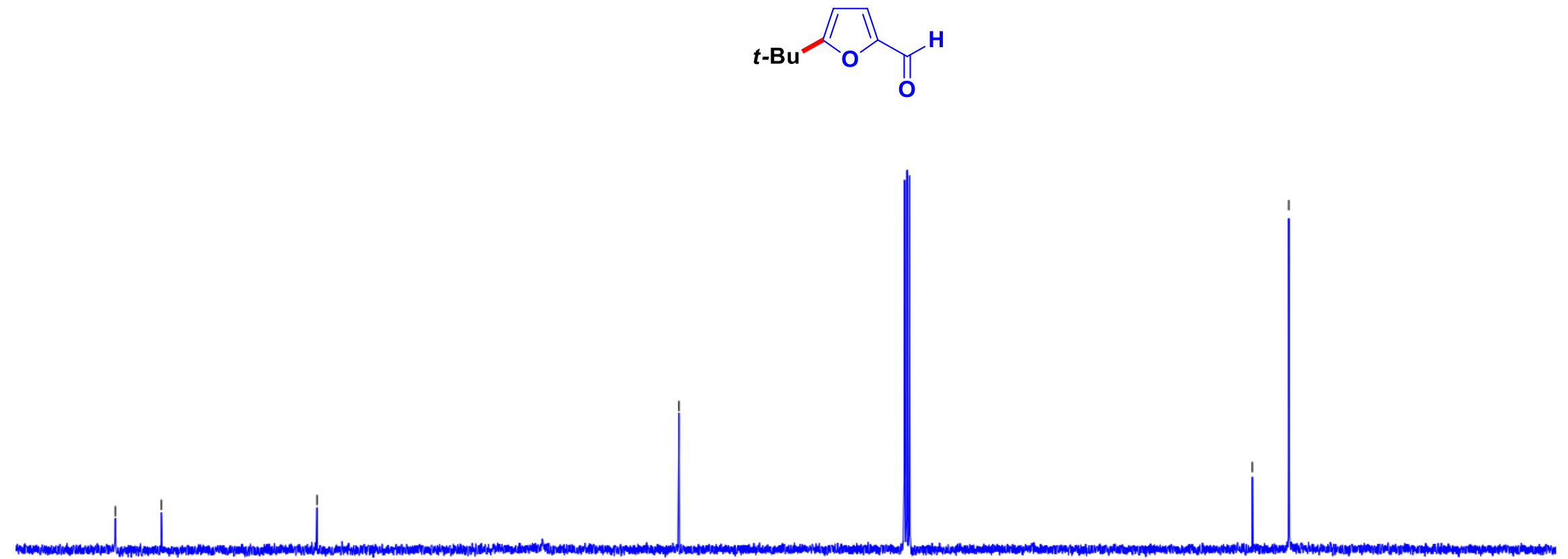

60

$150 \quad 140$

130

120

$110 \quad 100$

fl (ppm) 
Figure S-52. ${ }^{1} \mathrm{H}$ NMR spectrum of (1n) $\left(\mathrm{CDCl}_{3}, 400 \mathrm{MHz}\right)$ 1-(5-(tert-butyl)furan-2-yl)ethanone

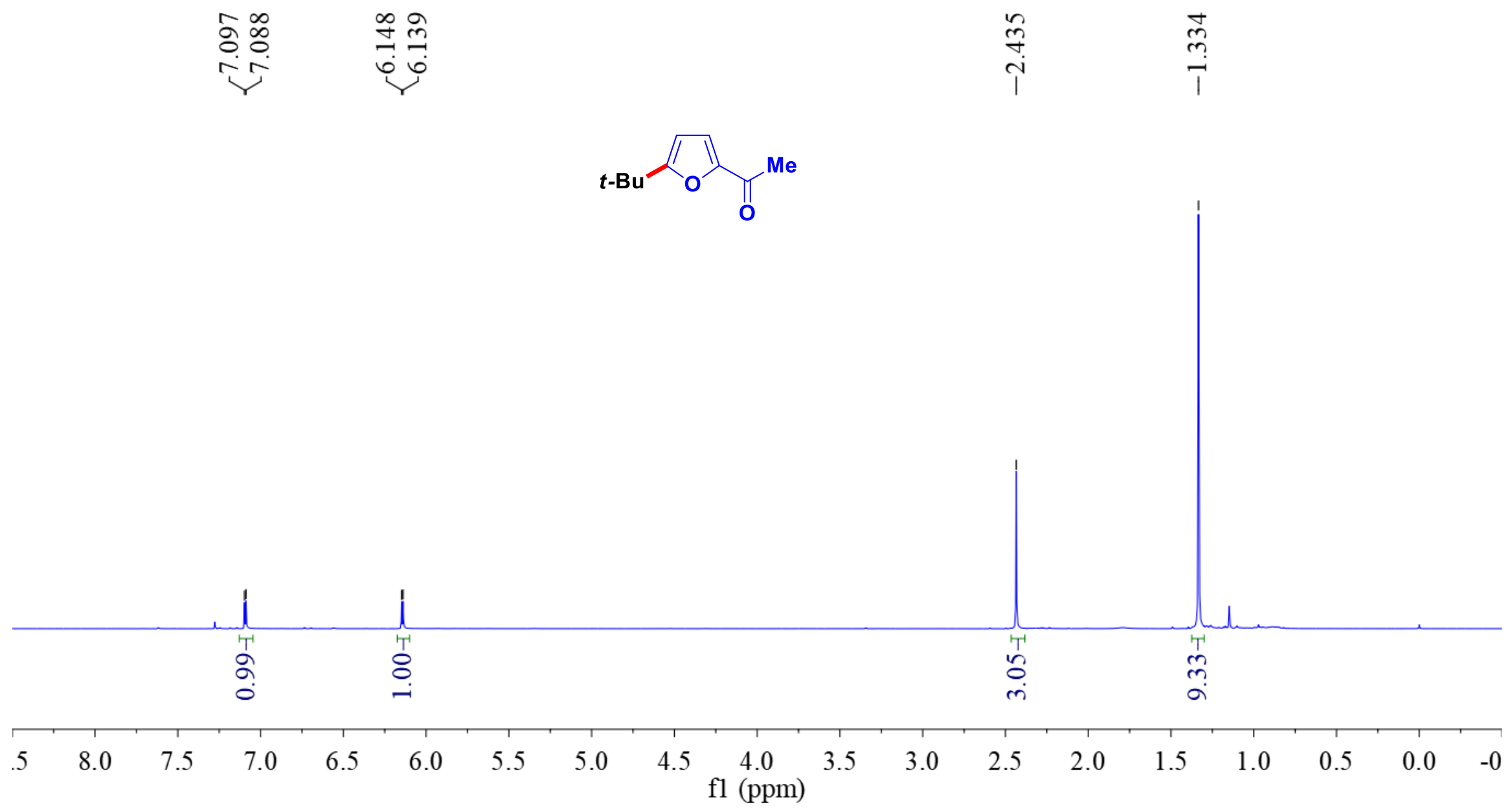


Figure S-53. ${ }^{13} \mathrm{C}\left\{{ }^{1} \mathrm{H}\right\} \mathrm{NMR}$ spectrum of (1n) $\left(\mathrm{CDCl}_{3}, 100 \mathrm{MHz}\right)$ 1-(5-(tert-butyl)furan-2-yl)ethanone

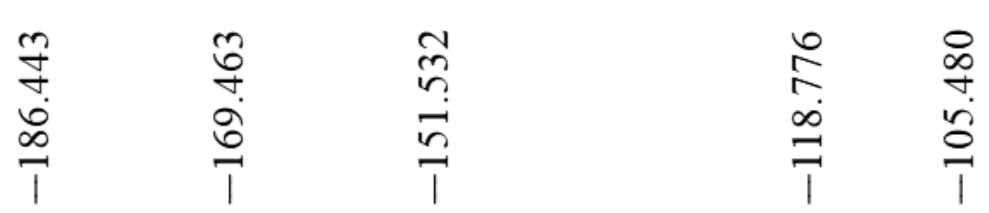

กิ

$\dddot{m} \stackrel{\infty}{1}$

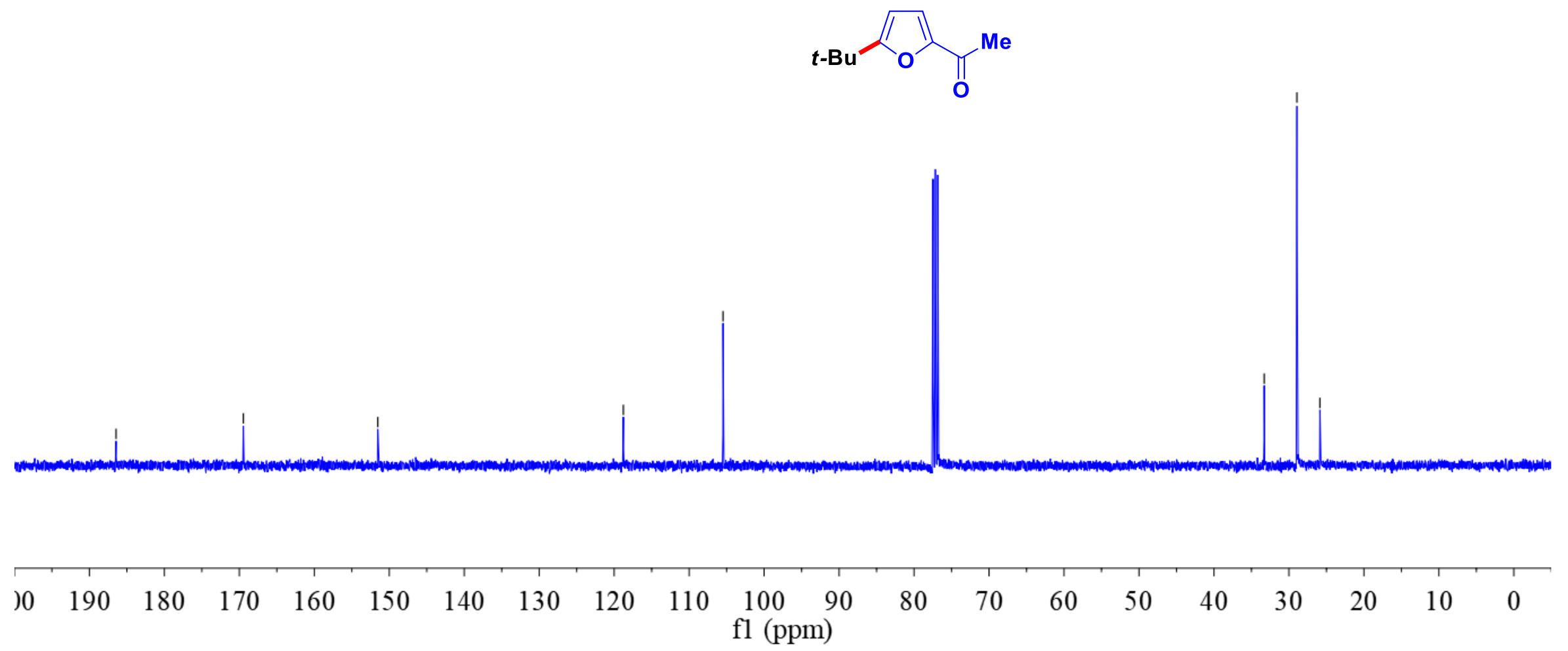


Figure S-54. ${ }^{1} \mathrm{H}$ NMR spectrum of (1o) $\left(\mathrm{CDCl}_{3}, 400 \mathrm{MHz}\right)$ 1-(5-(tert-butyl)furan-2-yl)-2,2-dimethylpropan-1-one 命永
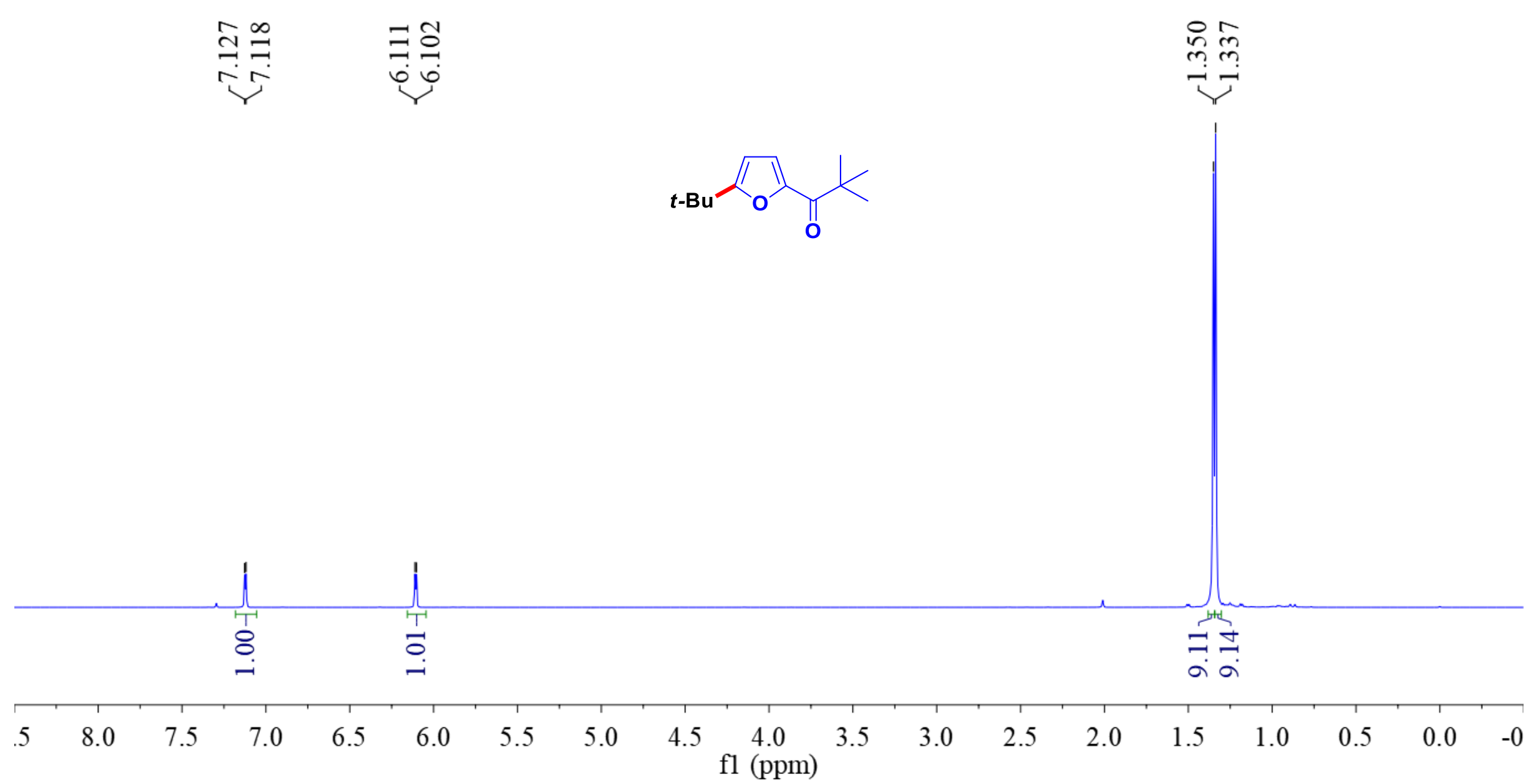
Figure S-55. ${ }^{13} \mathrm{C}\left\{{ }^{1} \mathrm{H}\right\}$ NMR spectrum of (10) $\left(\mathrm{CDCl}_{3}, 100 \mathrm{MHz}\right)$ 1-(5-(tert-butyl)furan-2-yl)-2,2-dimethylpropan-1-one
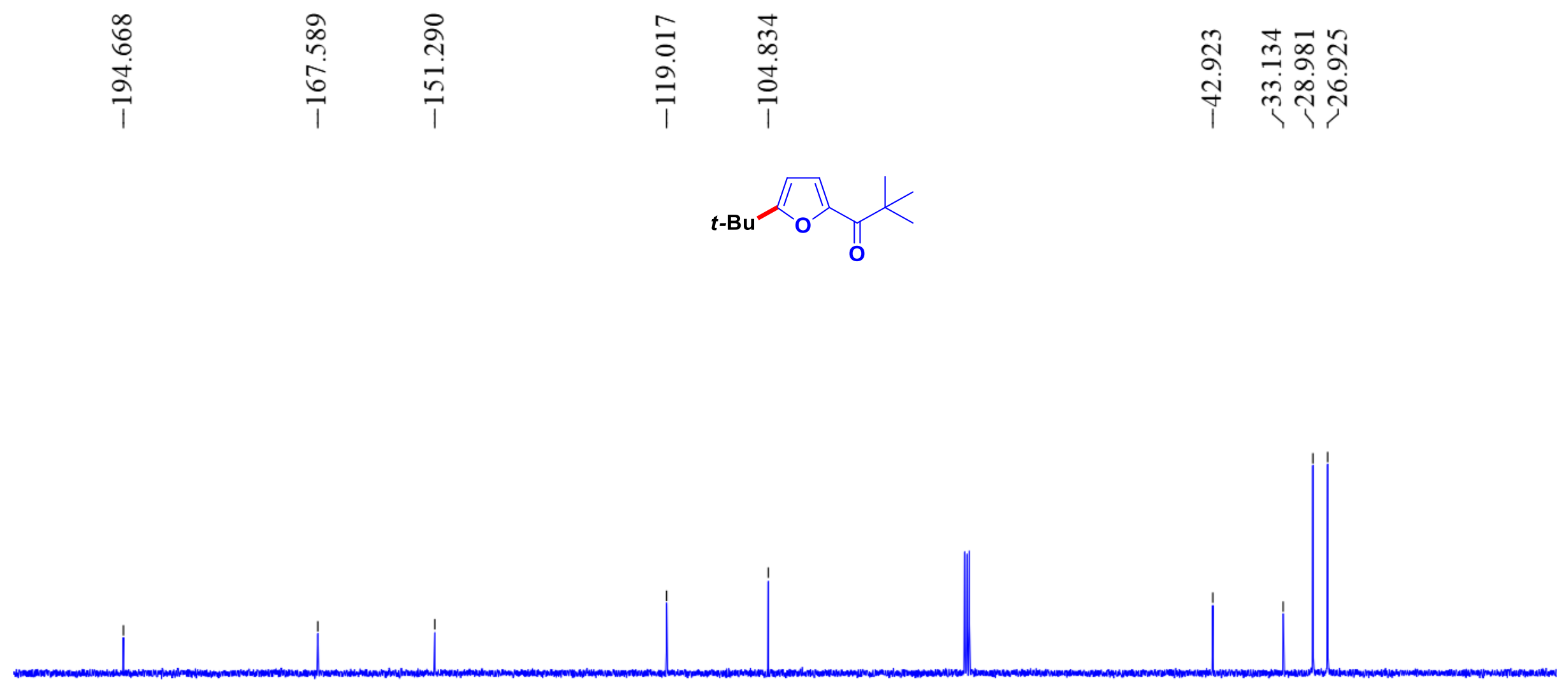

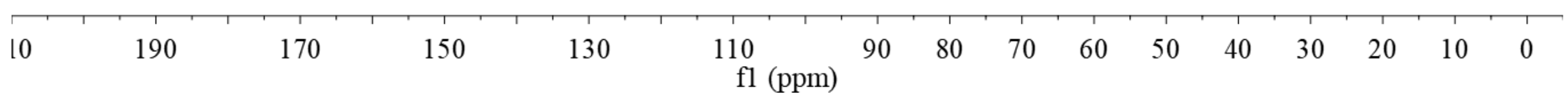


Figure S-56. ${ }^{1} \mathrm{H}$ NMR spectrum of (1p) $\left(\mathrm{CDCl}_{3}, 400 \mathrm{MHz}\right)$ 2-(tert-butyl)-5-phenylfuran

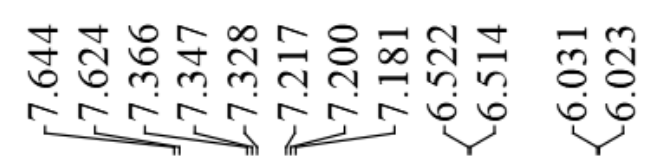

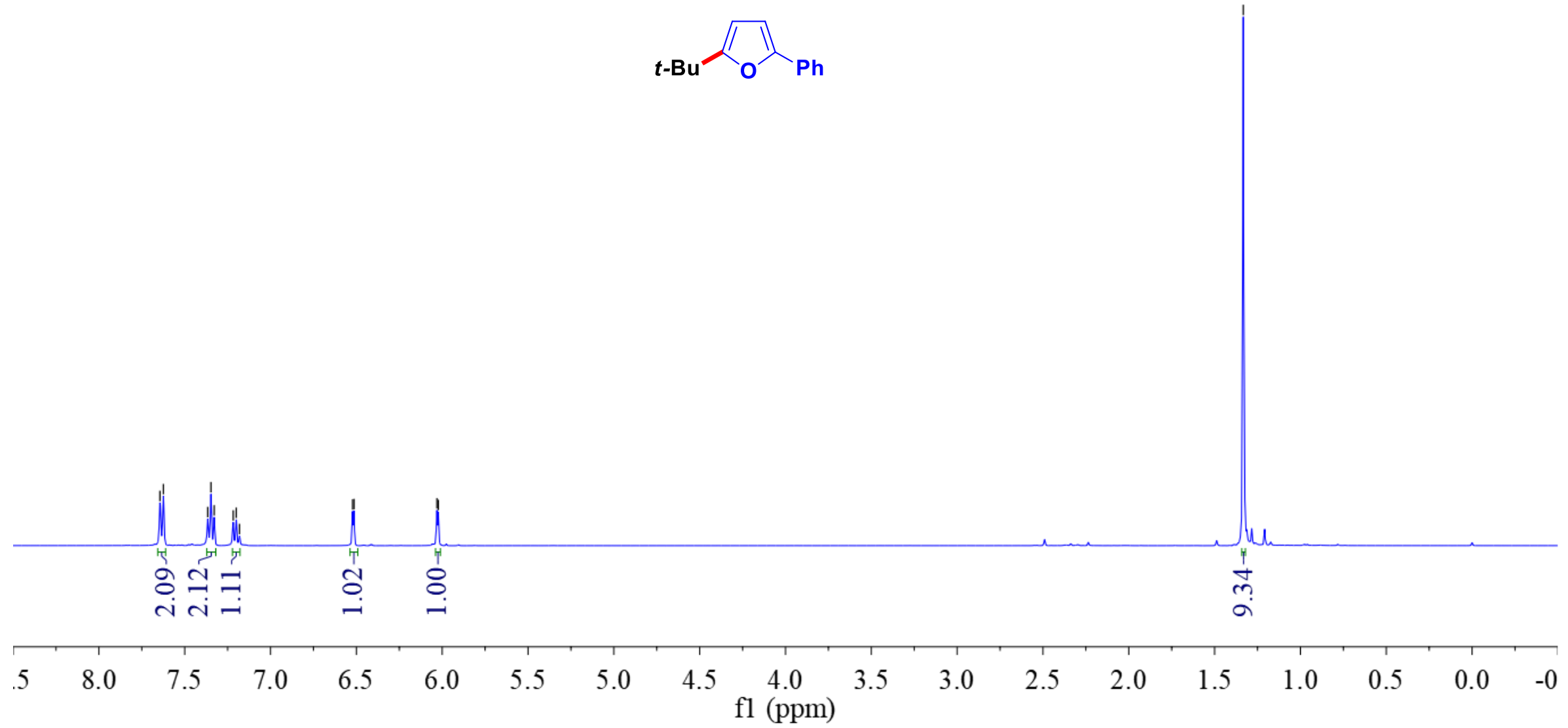


Figure S-57. ${ }^{13} \mathrm{C}\left\{{ }^{1} \mathrm{H}\right\}$ NMR spectrum of (1p) $\left(\mathrm{CDCl}_{3}, 100 \mathrm{MHz}\right)$ 2-(tert-butyl)-5-phenylfuran

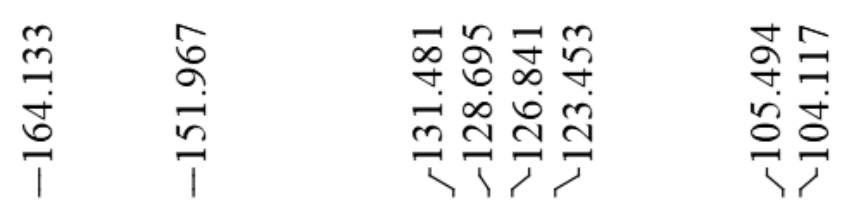

ลำ

กิ กิ

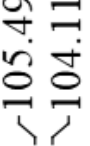
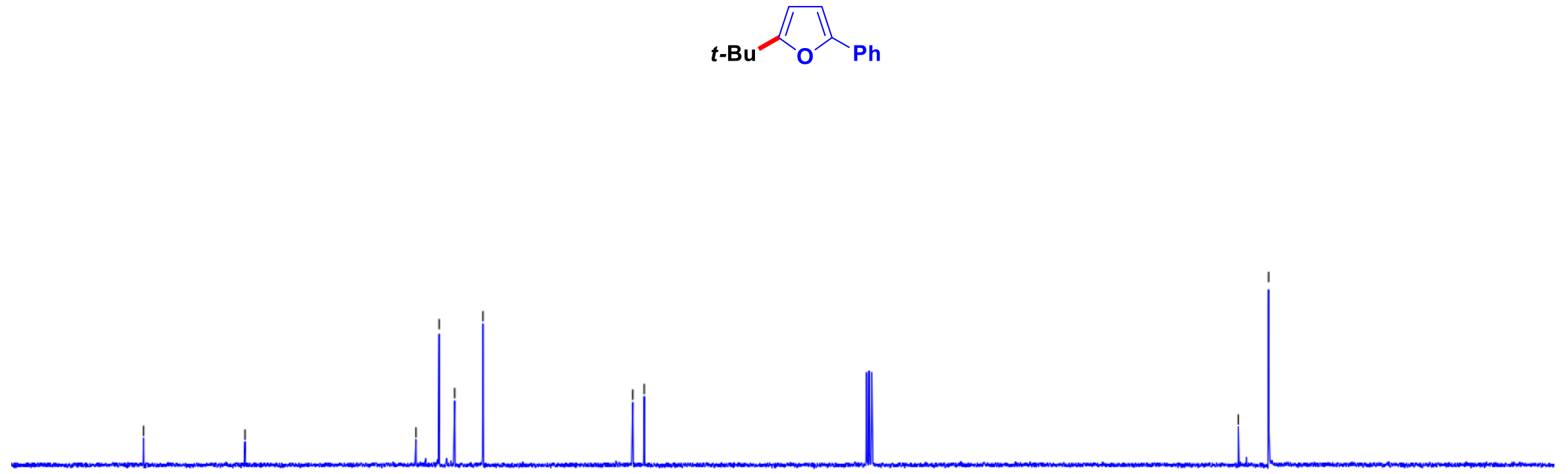

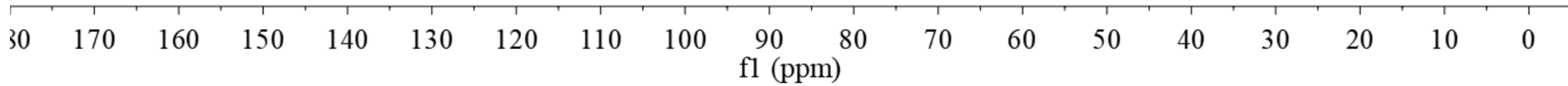


Figure S-58. ${ }^{1} \mathrm{H} \mathrm{NMR}$ spectrum of (1r) $\left(\mathrm{CDCl}_{3}, 400 \mathrm{MHz}\right)$ 2-(tert-butyl)benzofuran

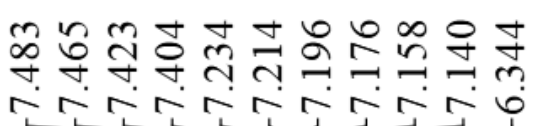

ก

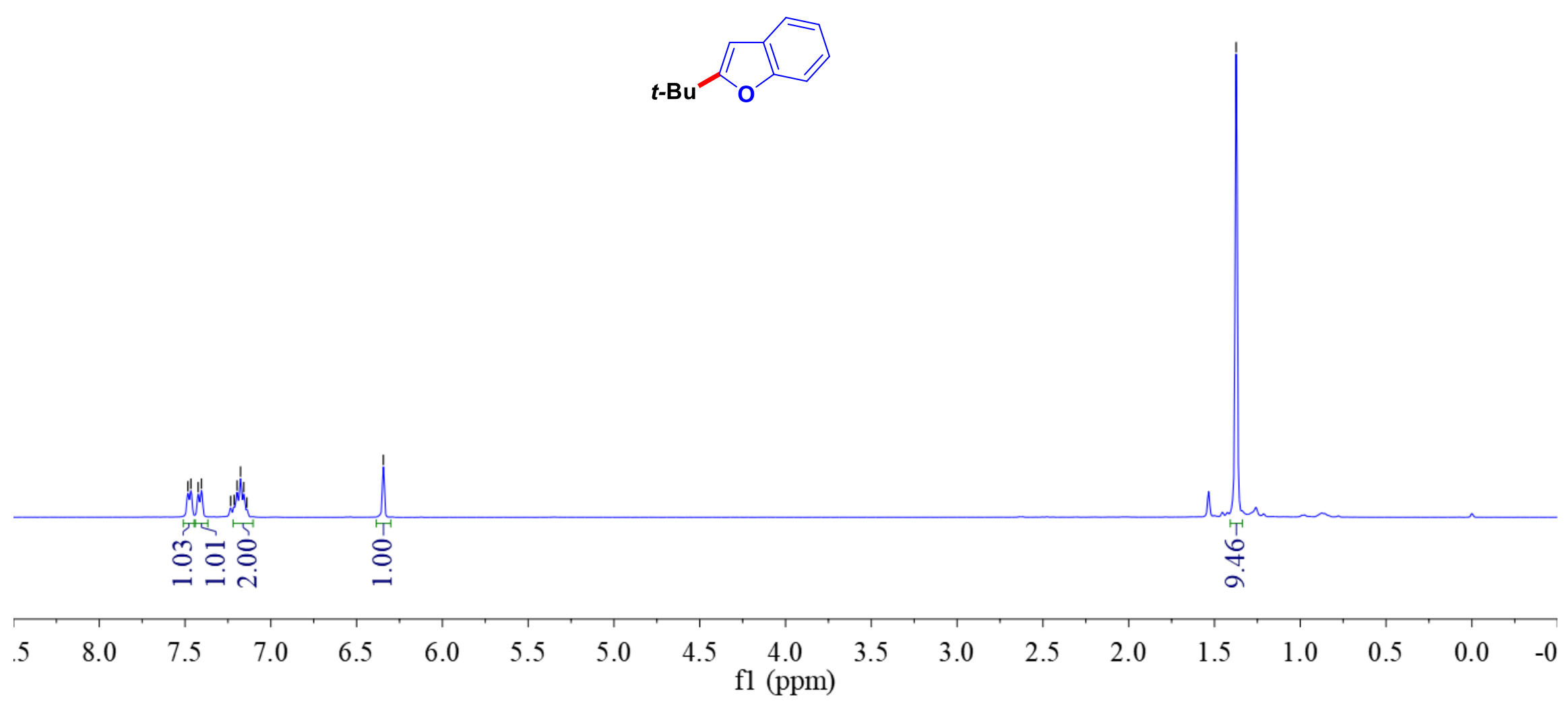


Figure S-59. ${ }^{13} \mathrm{C}\left\{{ }^{1} \mathrm{H}\right\}$ NMR spectrum of (1r) $\left(\mathrm{CDCl}_{3}, 100 \mathrm{MHz}\right)$ 2-(tert-butyl)benzofuran
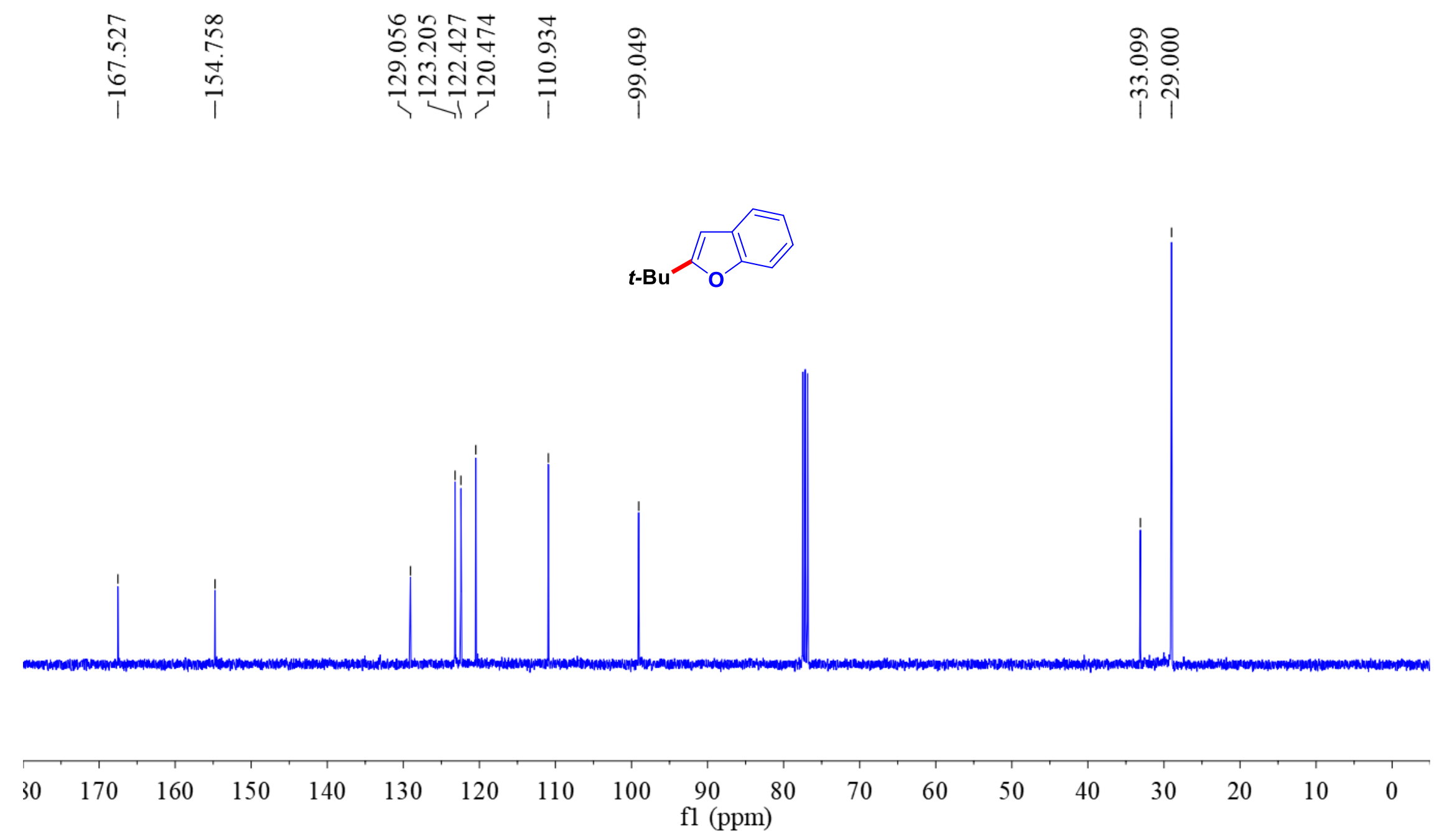
Figure S-60. ${ }^{1} \mathrm{H}$ NMR spectrum of (1s) $\left(\mathrm{CDCl}_{3}, 400 \mathrm{MHz}\right)$ 2,5-di-tert-butyl- $N$-methyl- $N$-phenylfuran-3-carboxamide
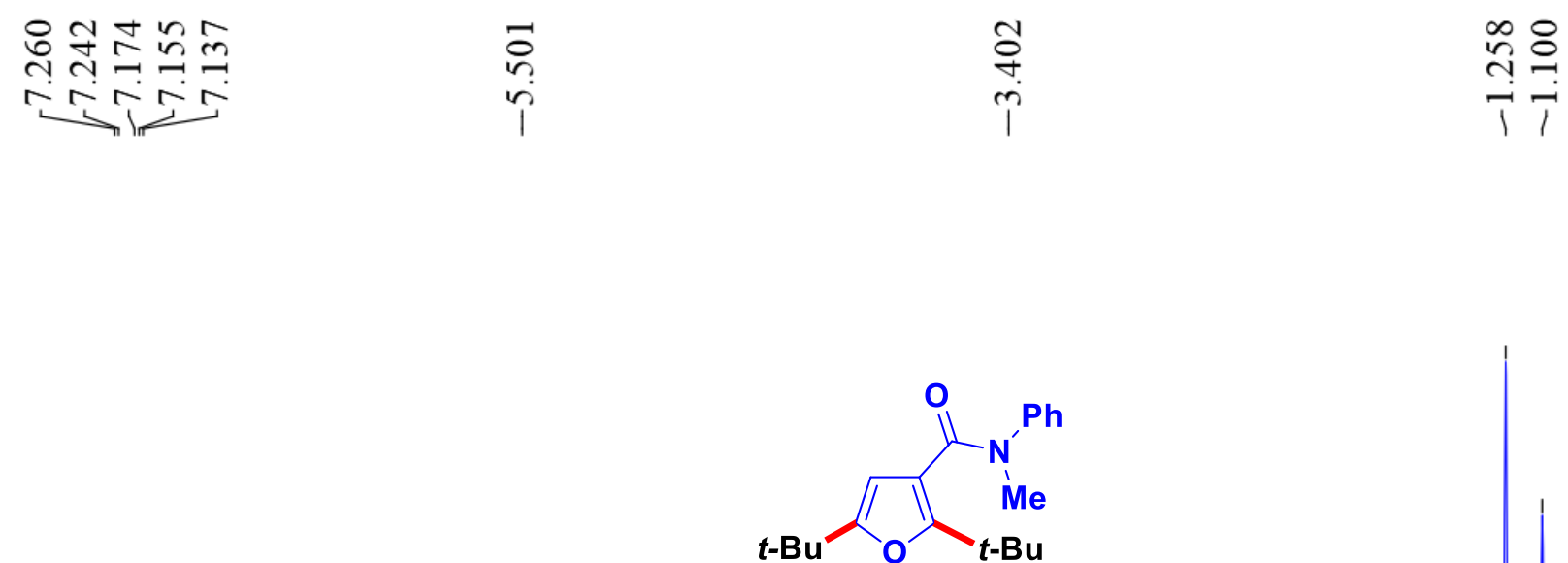

$$
\text { ヘே }
$$

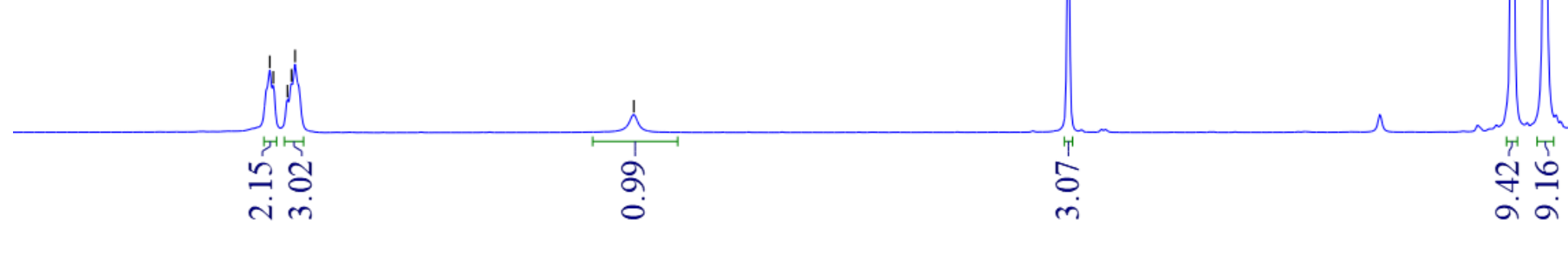

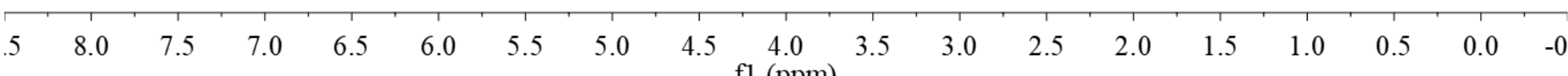


Figure S-61. ${ }^{13} \mathrm{C}\left\{{ }^{1} \mathrm{H}\right\}$ NMR spectrum of (1s) $\left(\mathrm{CDCl}_{3}, 100 \mathrm{MHz}\right)$ 2,5-di-tert-butyl- $N$-methyl- $\boldsymbol{N}$-phenylfuran-3-carboxamide

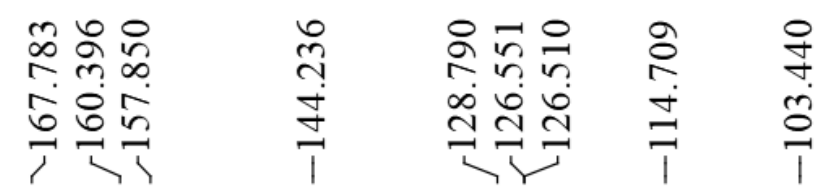

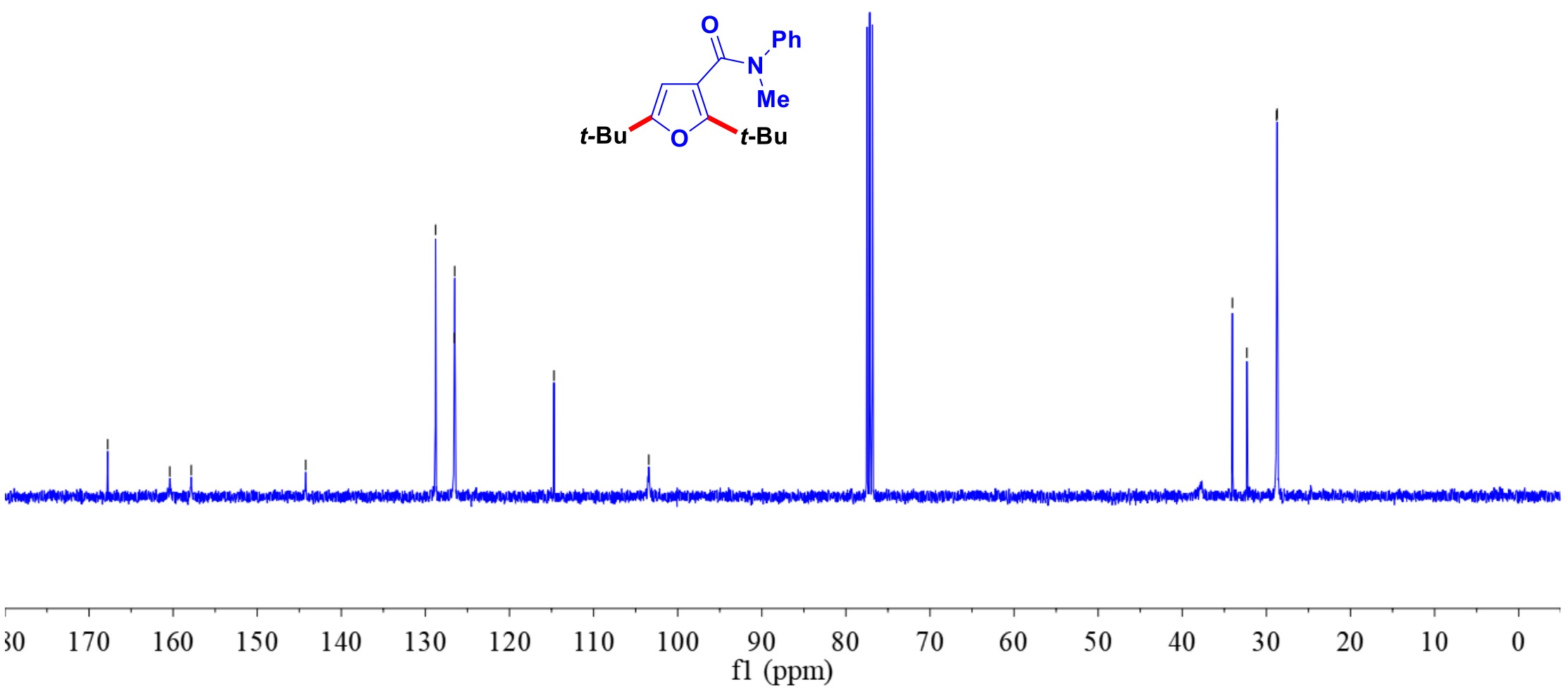


Figure S-62. ${ }^{1} \mathrm{H}$ NMR spectrum of (1t) $\left(\mathrm{CDCl}_{3}, 400 \mathrm{MHz}\right)$ 5-(1-methylcyclohexyl)- $N$-methyl- $N$-phenylfuran-2-carboxamide

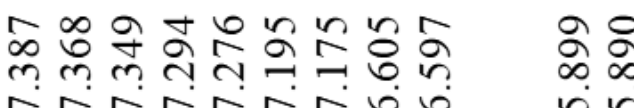
तrrárion
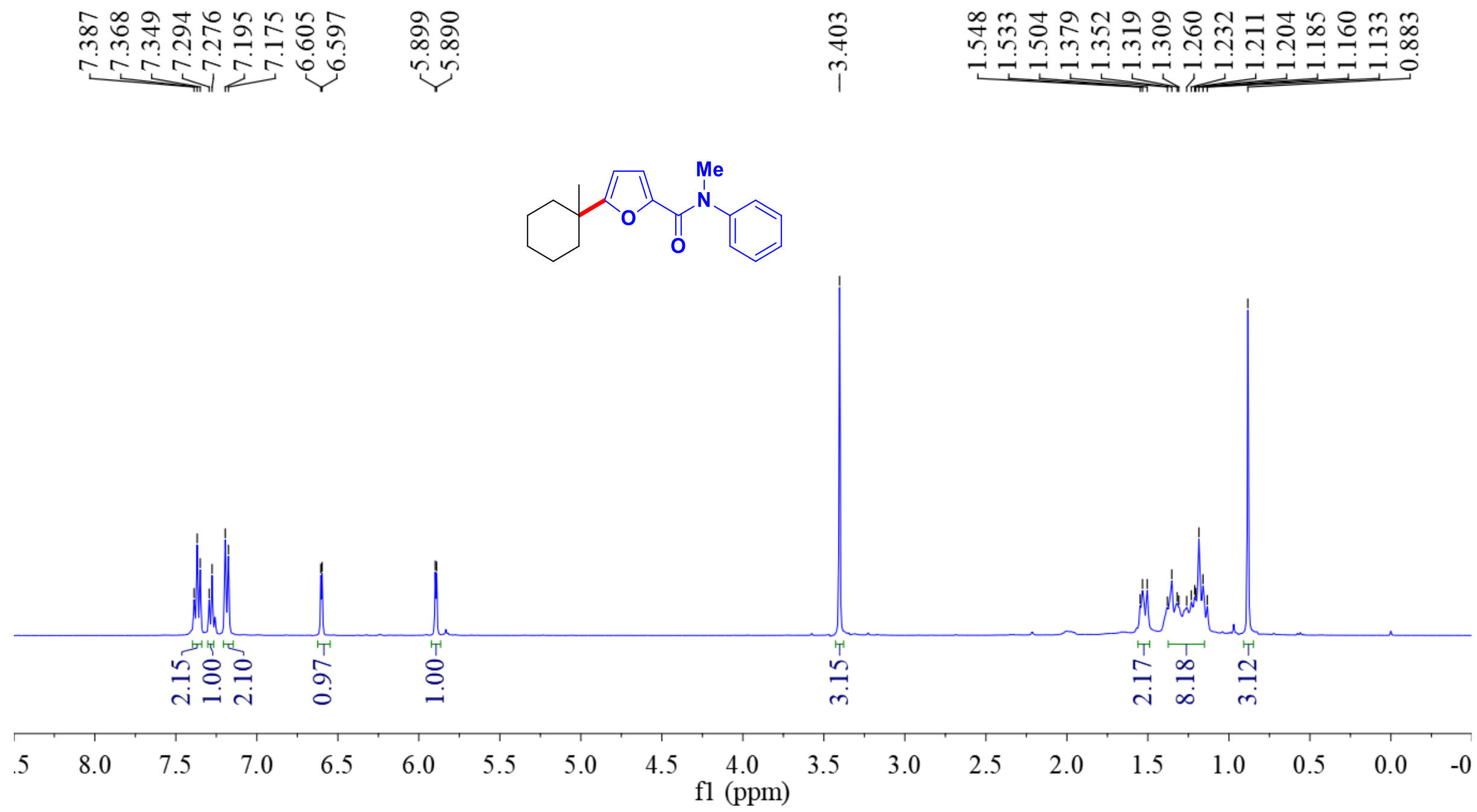
Figure S-63. ${ }^{13} \mathrm{C}\left\{{ }^{1} \mathrm{H}\right\}$ NMR spectrum of (1t) $\left(\mathrm{CDCl}_{3}, 100 \mathrm{MHz}\right)$ 5-(1-methylcyclohexyl)- $N$-methyl- $N$-phenylfuran-2-carboxamide

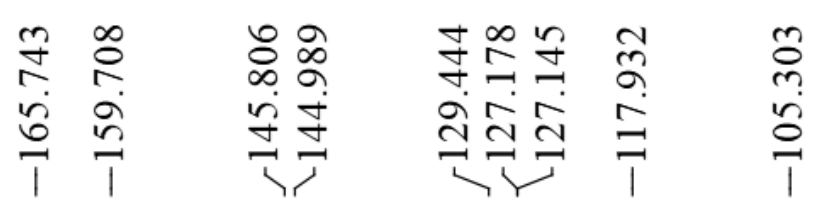

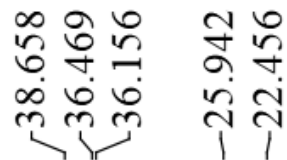

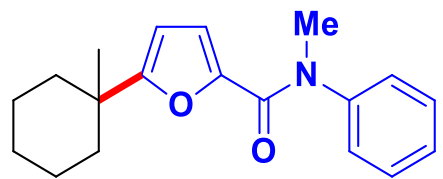

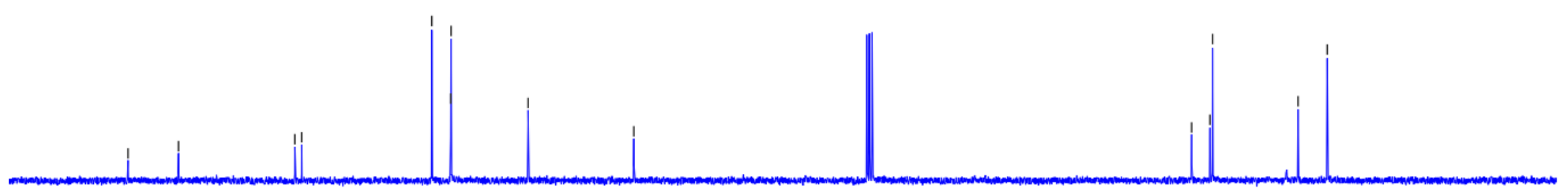

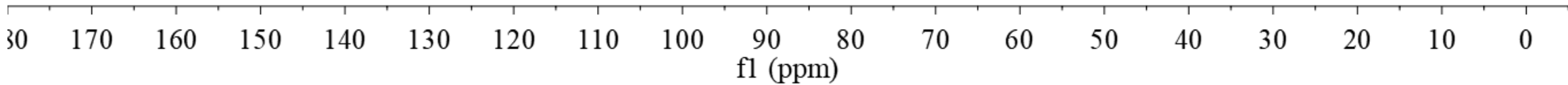


Figure S-64. ${ }^{1} \mathrm{H}$ NMR spectrum of (1u) $\left(\mathrm{CDCl}_{3}, 400 \mathrm{MHz}\right)$ 5-(1-(4-isopropylphenyl)propan-2-yl)- $N$-methyl- $N$-phenylfuran-2-carboxamide

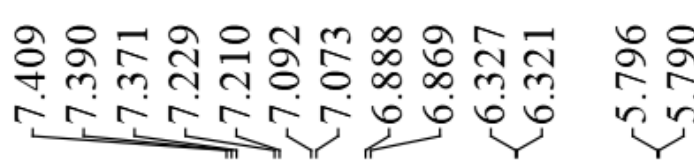

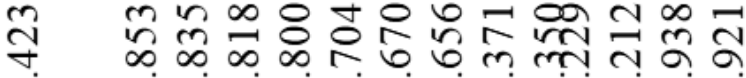

m Nuriariario
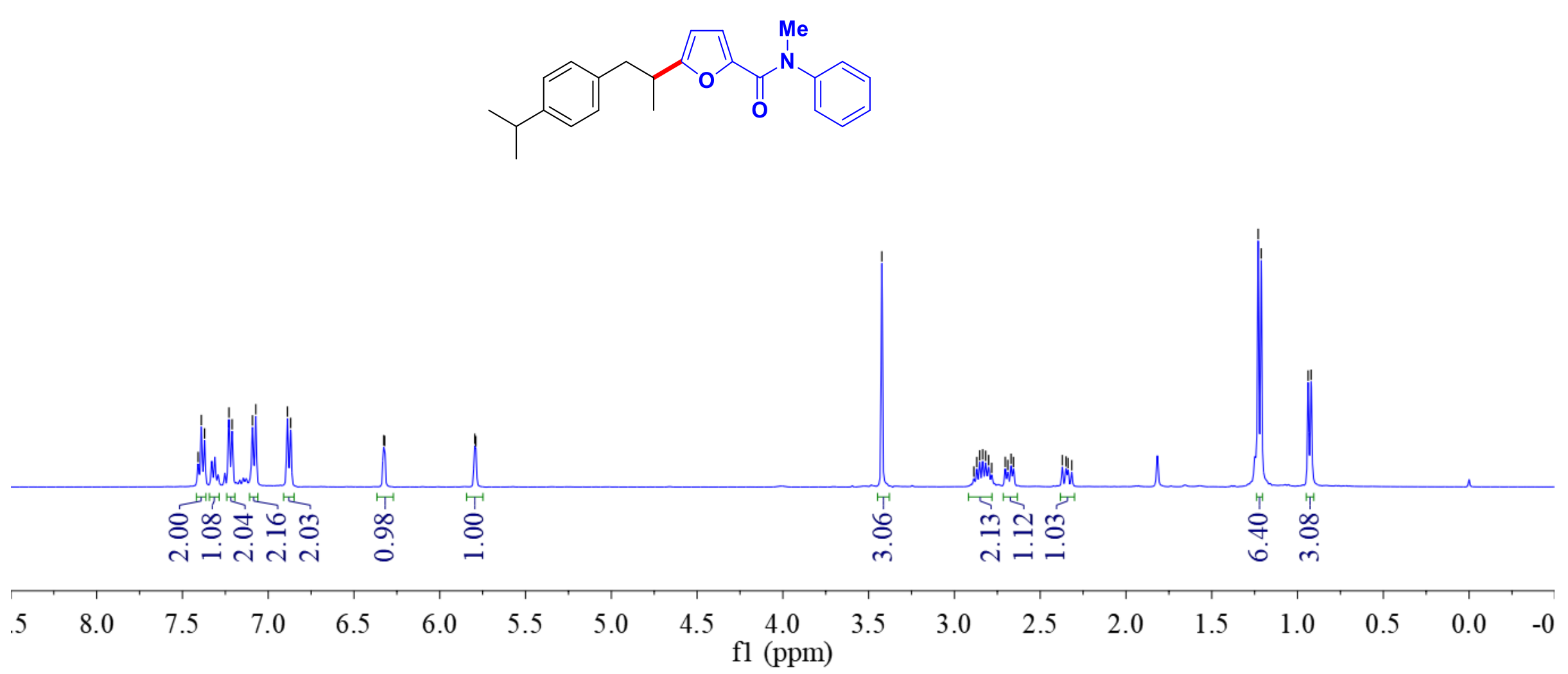
Figure S-65. ${ }^{13} \mathrm{C}\left\{{ }^{1} \mathrm{H}\right\}$ NMR spectrum of (1u) $\left(\mathrm{CDCl}_{3}, 100 \mathrm{MHz}\right)$ 5-(1-(4-isopropylphenyl)propan-2-yl)- $N$-methyl- $N$-phenylfuran-2-carboxamide

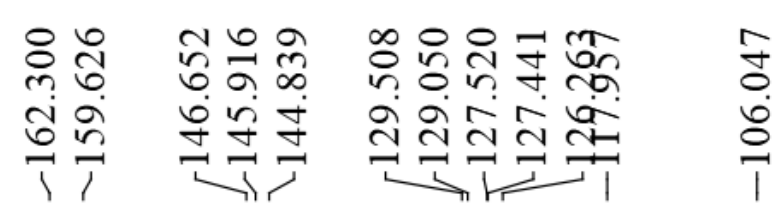

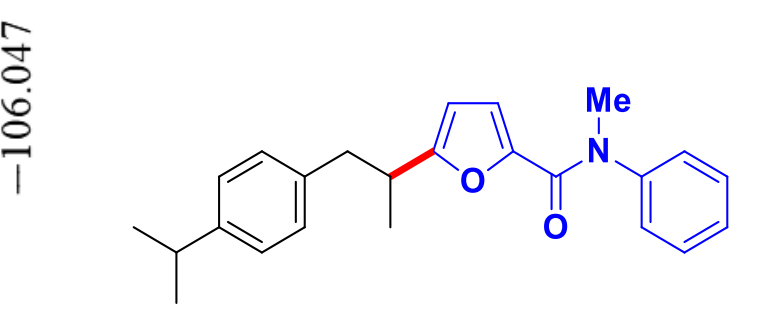

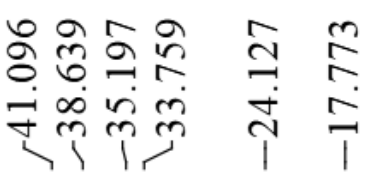

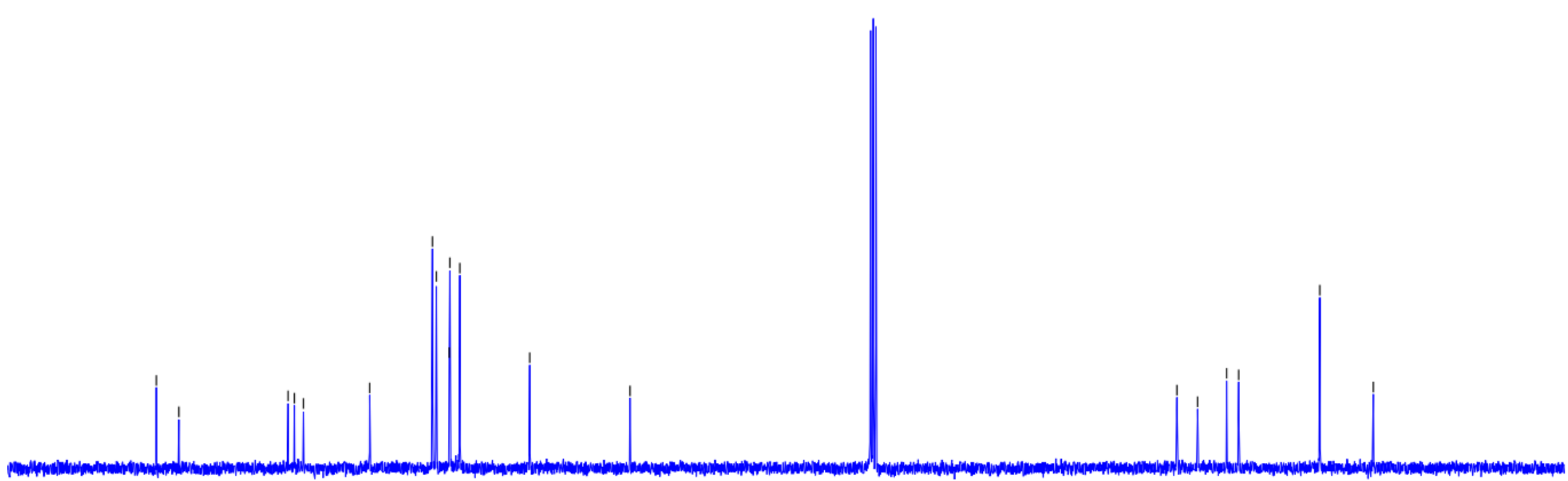

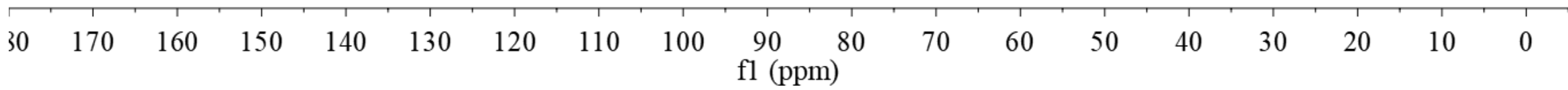


Figure S-66. ${ }^{1} \mathrm{H}$ NMR spectrum of (1v) $\left(\mathrm{CDCl}_{3}, 400 \mathrm{MHz}\right)$ 5-(1-(4-(tert-butyl)phenyl)propan-2-yl)- $N$-methyl- $N$-phenylfuran-2-carboxamide

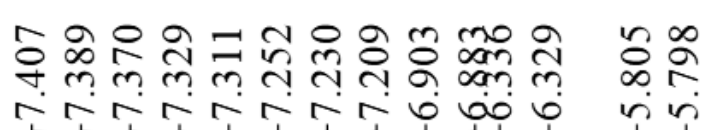
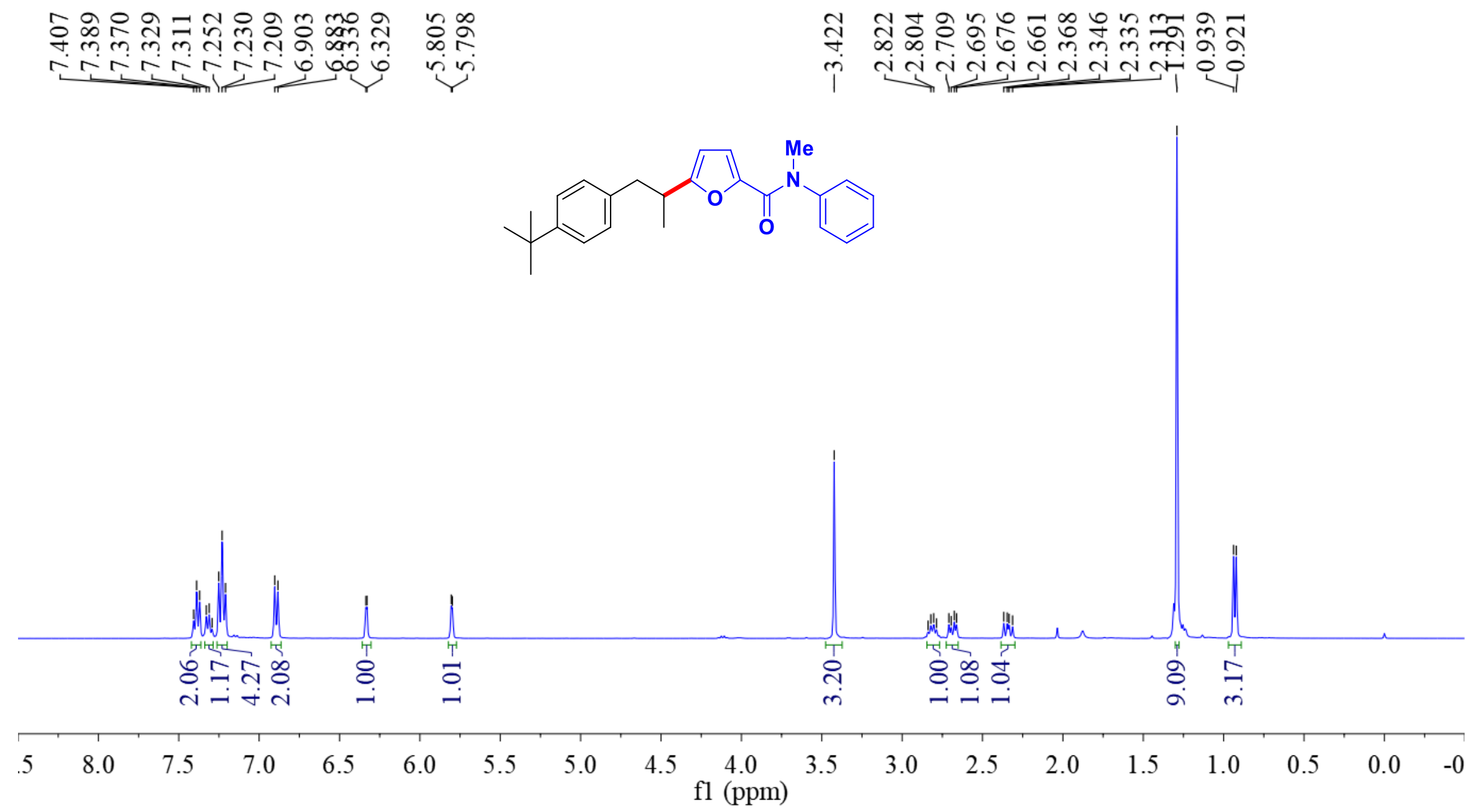
Figure S-67. ${ }^{13} \mathrm{C}\left\{{ }^{1} \mathrm{H}\right\}$ NMR spectrum of (1v) $\left(\mathrm{CDCl}_{3}, 100 \mathrm{MHz}\right)$ 5-(1-(4-(tert-butyl)phenyl)propan-2-yl)- $N$-methyl- $N$-phenylfuran-2-carboxamide
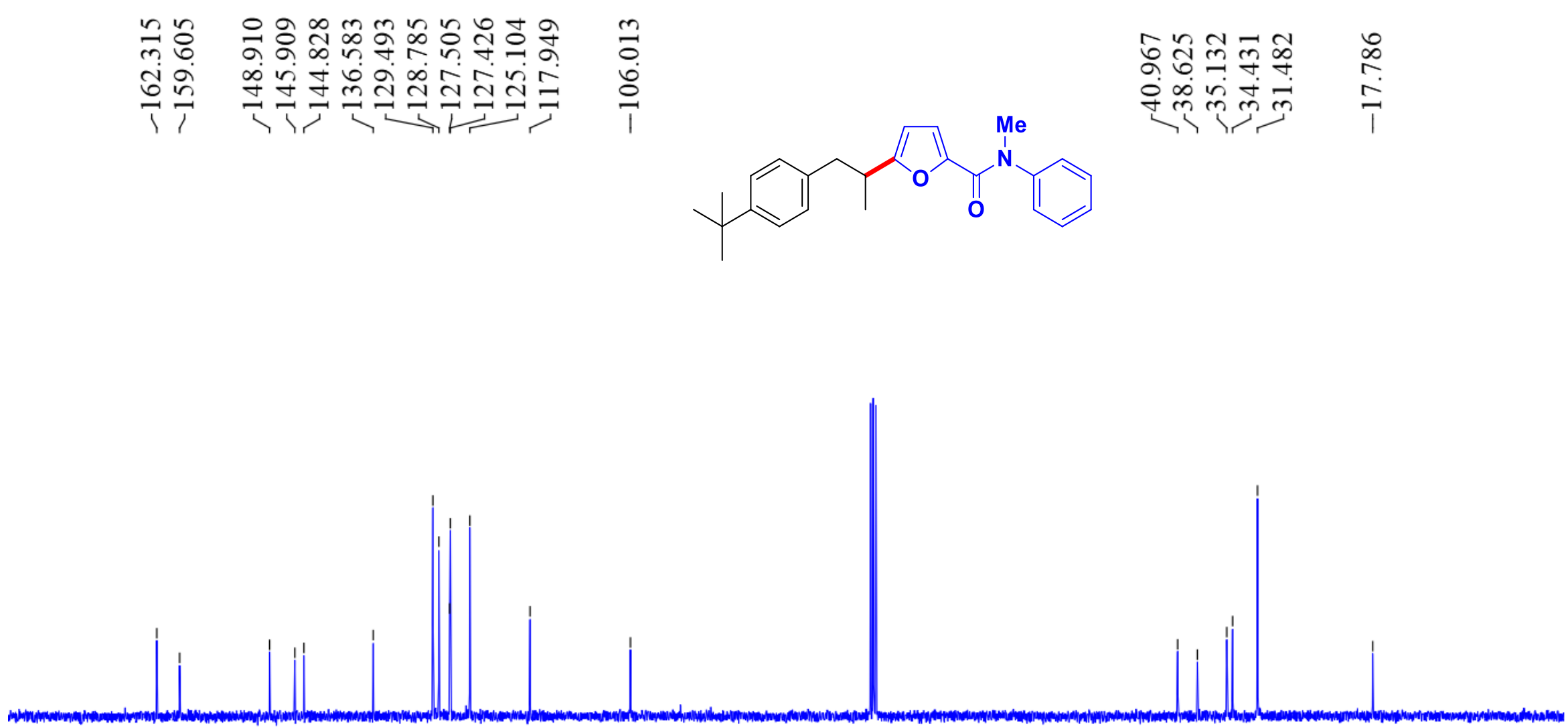
Figure S-68. ${ }^{1} \mathrm{H}$ NMR spectrum of $(\mathbf{1 w})\left(\mathrm{CDCl}_{3}, 400 \mathrm{MHz}\right)$ 5-cyclohexyl- $N$-methyl- $N$-phenylfuran-2-carboxamide

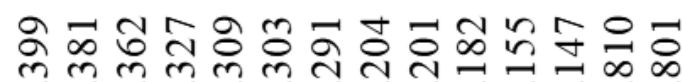
लrin

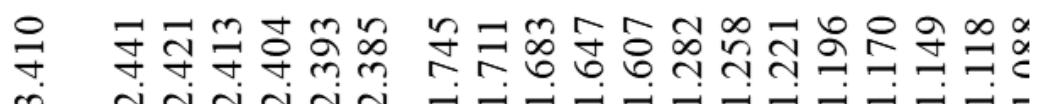

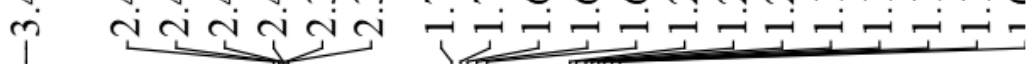

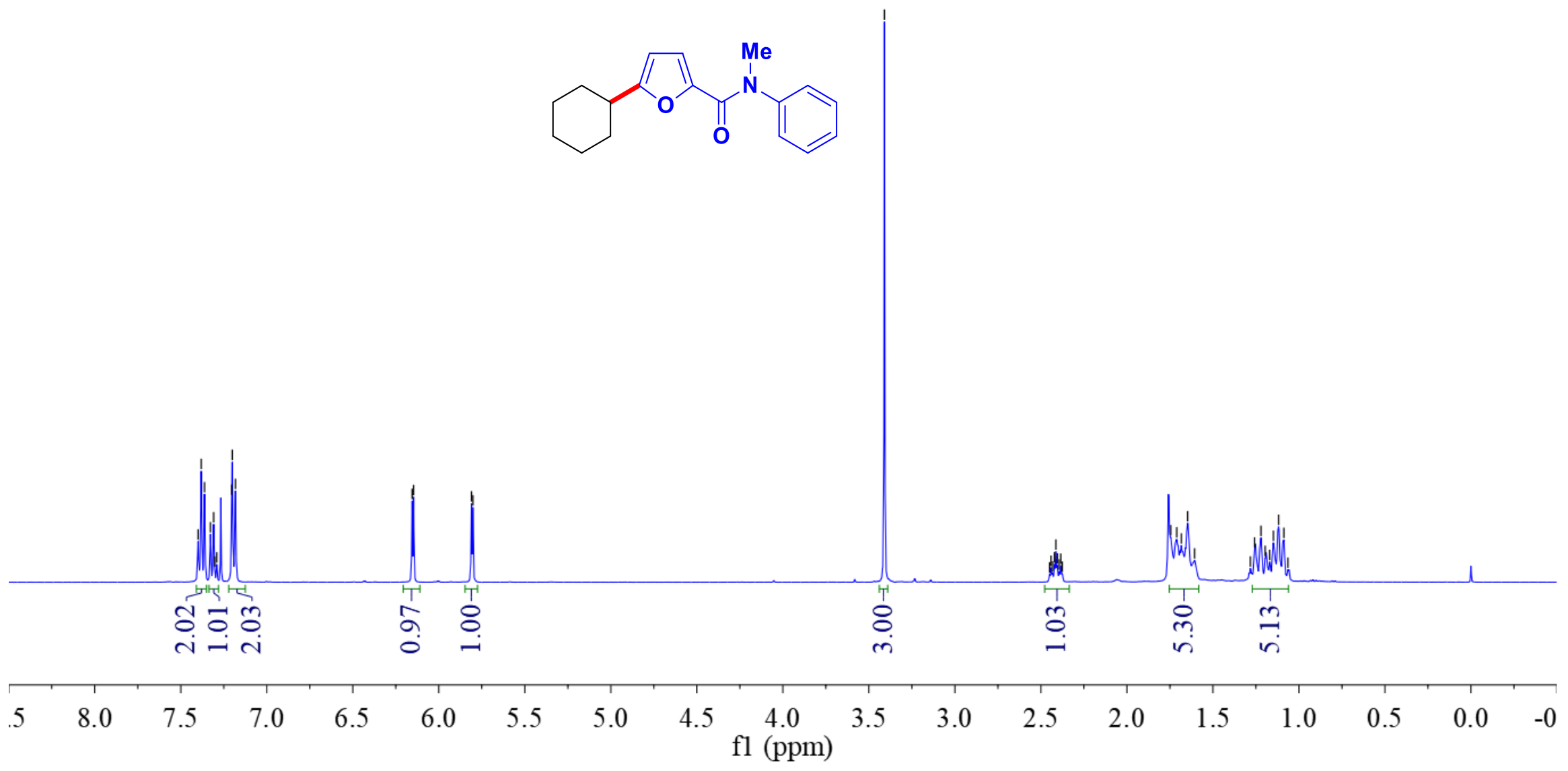


Figure S-69. ${ }^{13} \mathrm{C}\left\{{ }^{1} \mathrm{H}\right\}$ NMR spectrum of (1w) $\left(\mathrm{CDCl}_{3}, 100 \mathrm{MHz}\right)$ 5-cyclohexyl- $N$-methyl- $N$-phenylfuran-2-carboxamide

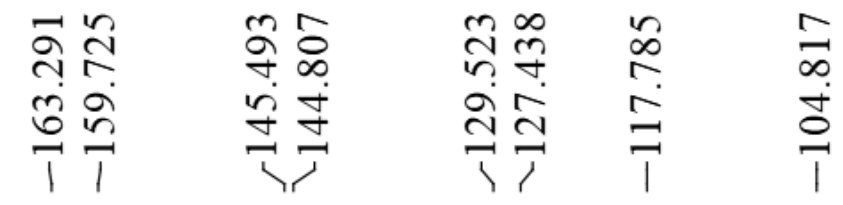
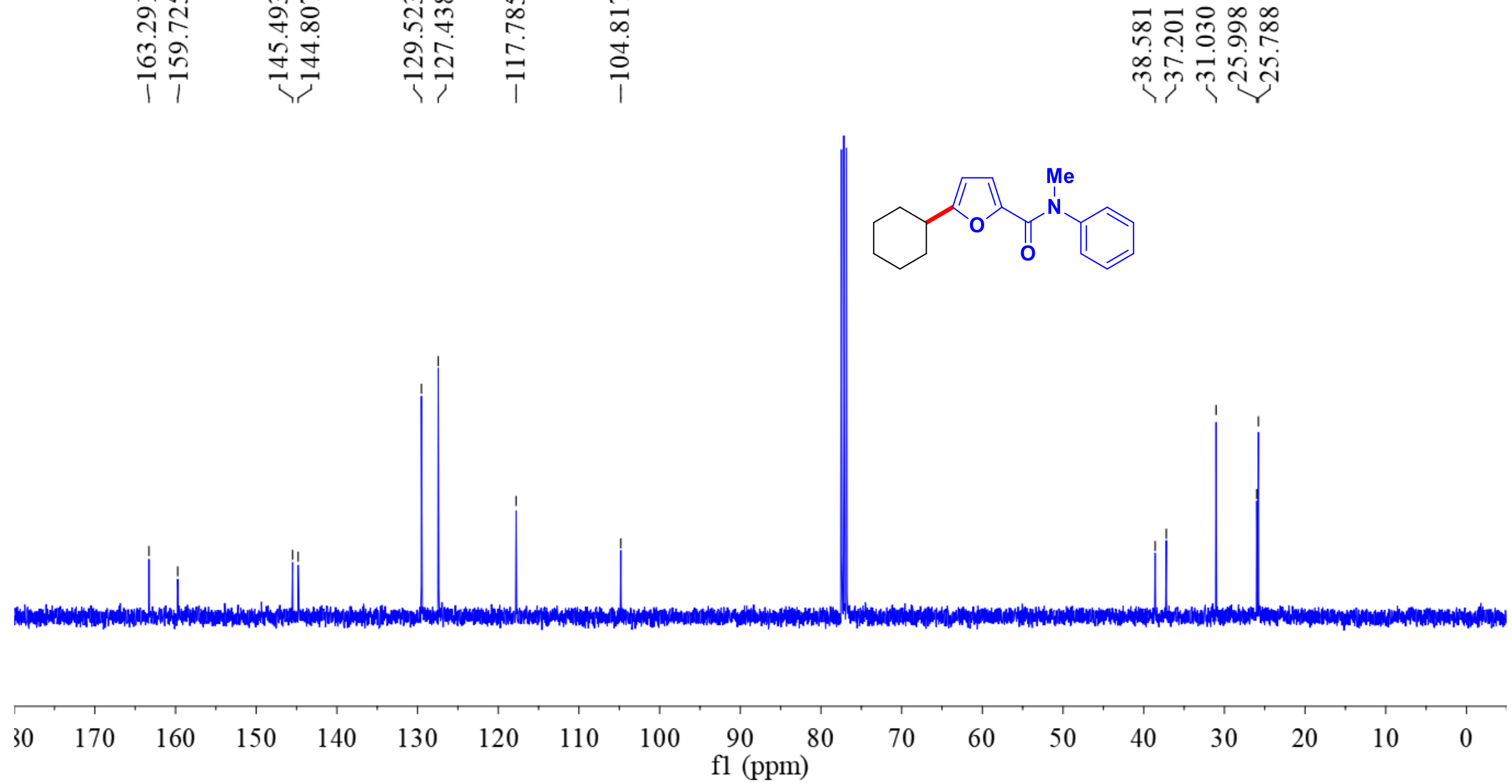
Figure S-70. ${ }^{1} \mathrm{H}$ NMR spectrum of (1x) $\left(\mathrm{CDCl}_{3}, 400 \mathrm{MHz}\right)$ 5-cyclopentyl- $N$-methyl- $N$-phenylfuran-2-carboxamide

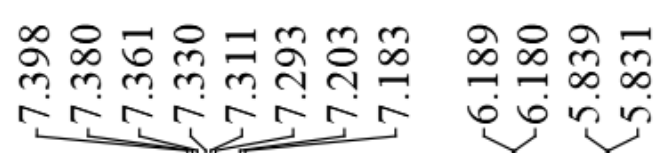
깅 $\exists \sigma \infty \infty \infty \infty \pi)$

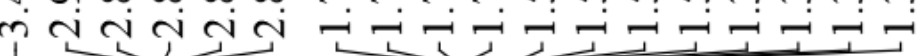

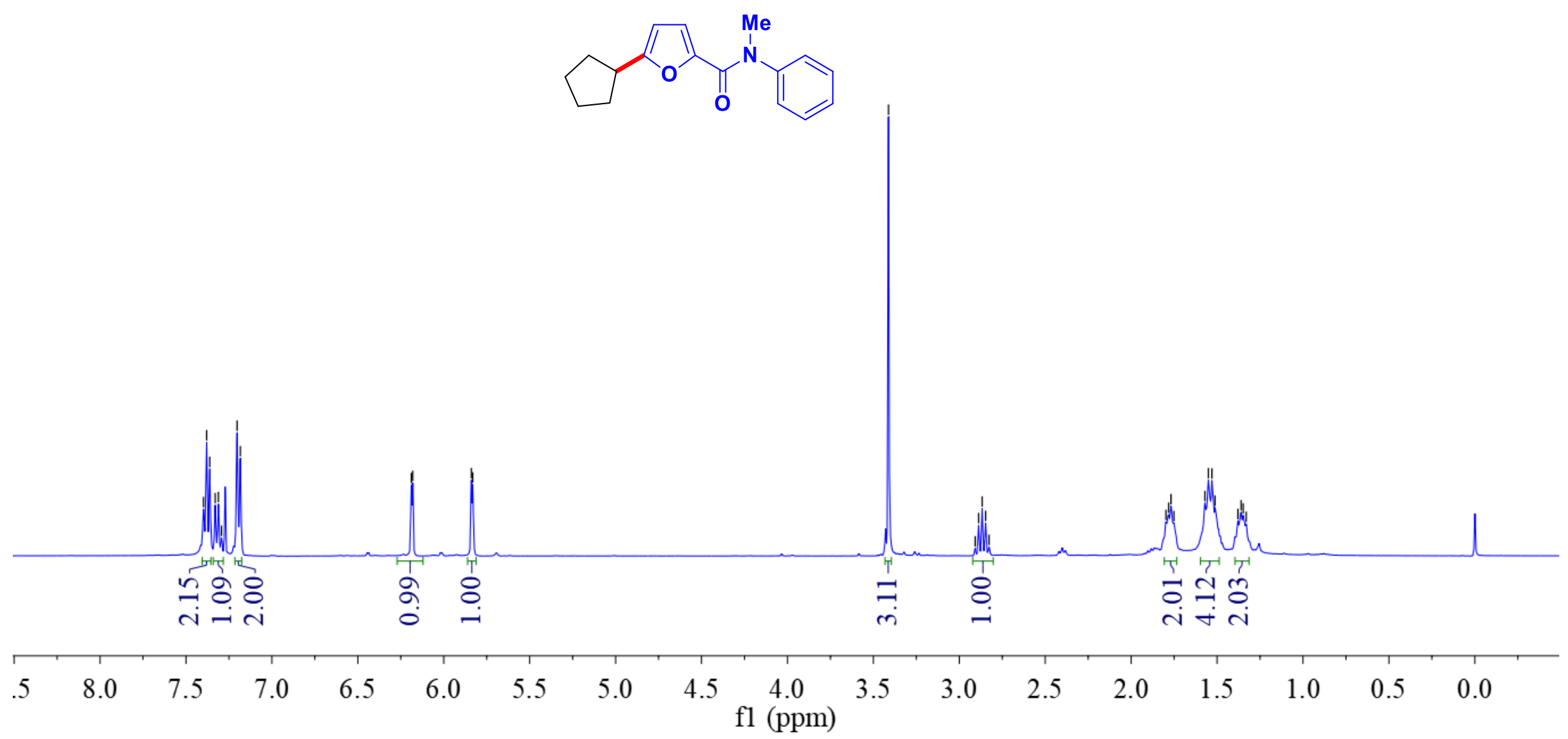


Figure S-71. ${ }^{13} \mathrm{C}\left\{{ }^{1} \mathrm{H}\right\}$ NMR spectrum of $(\mathbf{1 x})\left(\mathrm{CDCl}_{3}, 100 \mathrm{MHz}\right)$ 5-cyclopentyl- $N$-methyl- $N$-phenylfuran-2-carboxamide

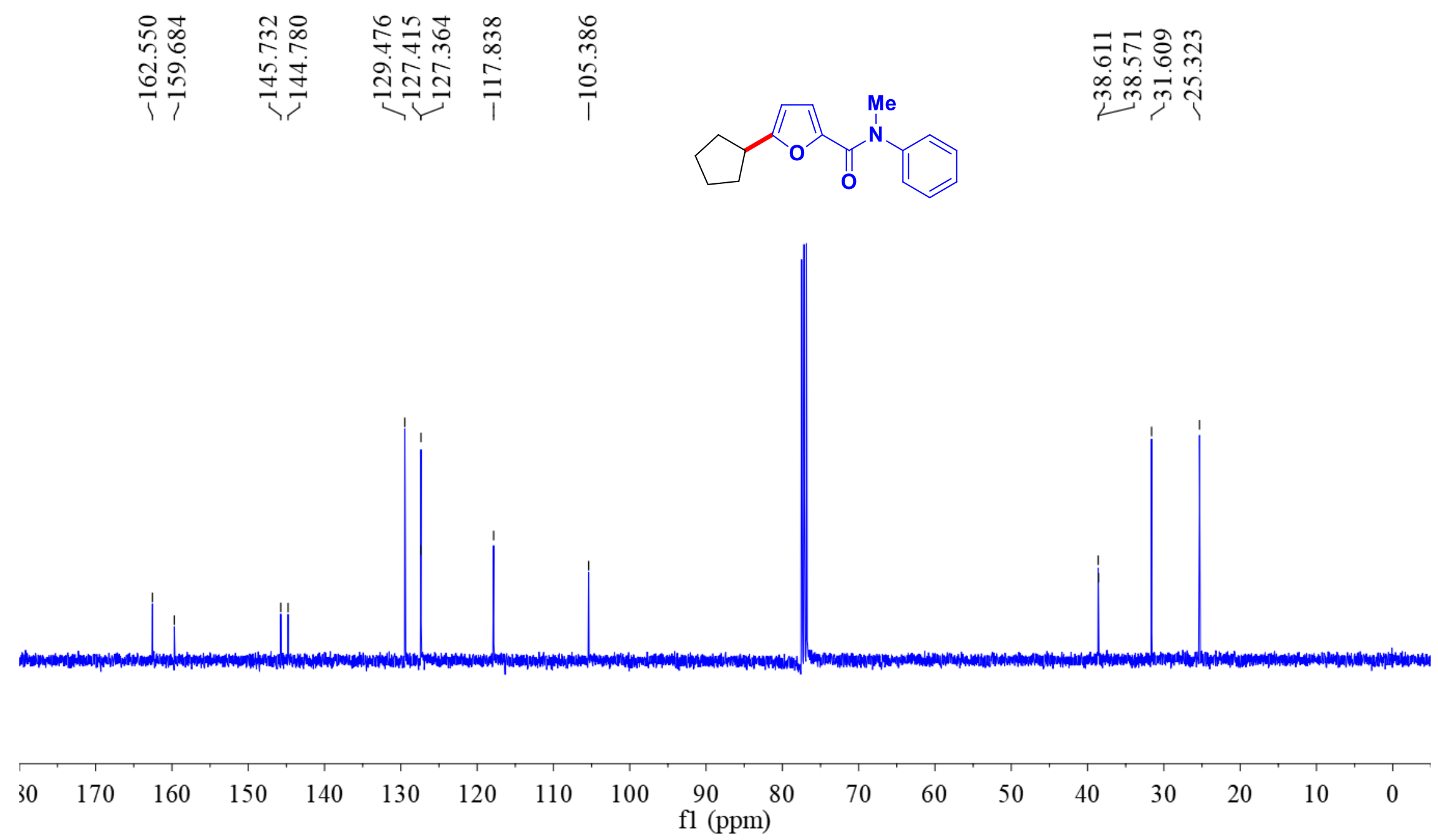


Figure S-72. ${ }^{1} \mathrm{H}$ NMR spectrum of (1y) $\left(\mathrm{CDCl}_{3}, 400 \mathrm{MHz}\right)$ 5-(cyclohex-3-en-1-yl)- $N$-methyl- $N$-phenylfuran-2-carboxamide
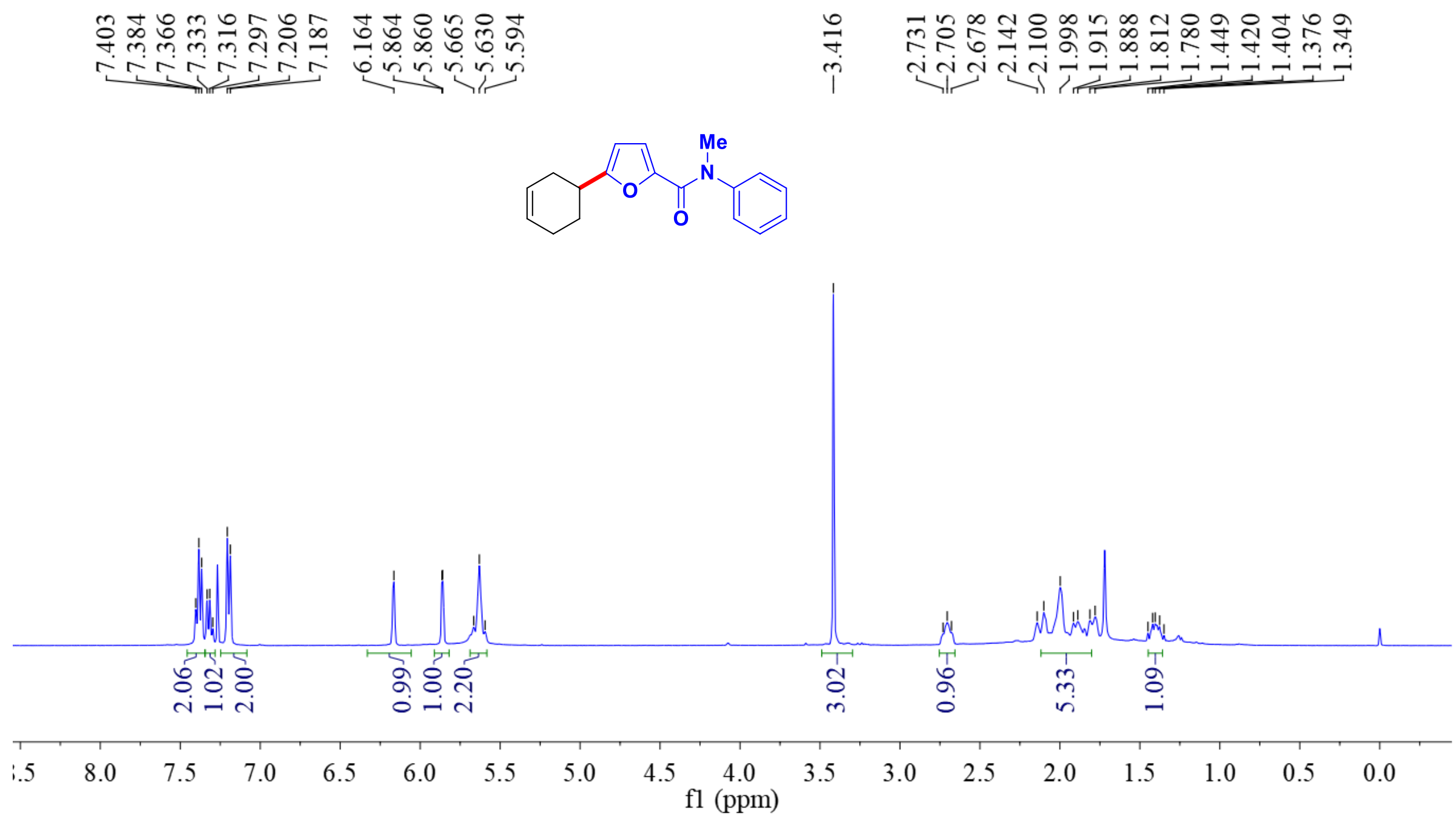
Figure S-73. ${ }^{13} \mathrm{C}\left\{{ }^{1} \mathrm{H}\right\}$ NMR spectrum of (1y) $\left(\mathrm{CDCl}_{3}, 100 \mathrm{MHz}\right)$ 5-(cyclohex-3-en-1-yl)- $N$-methyl- $N$-phenylfuran-2-carboxamide

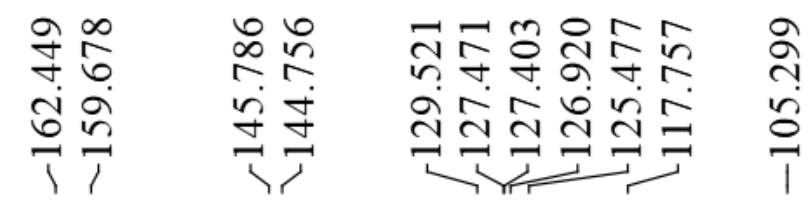

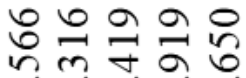
m

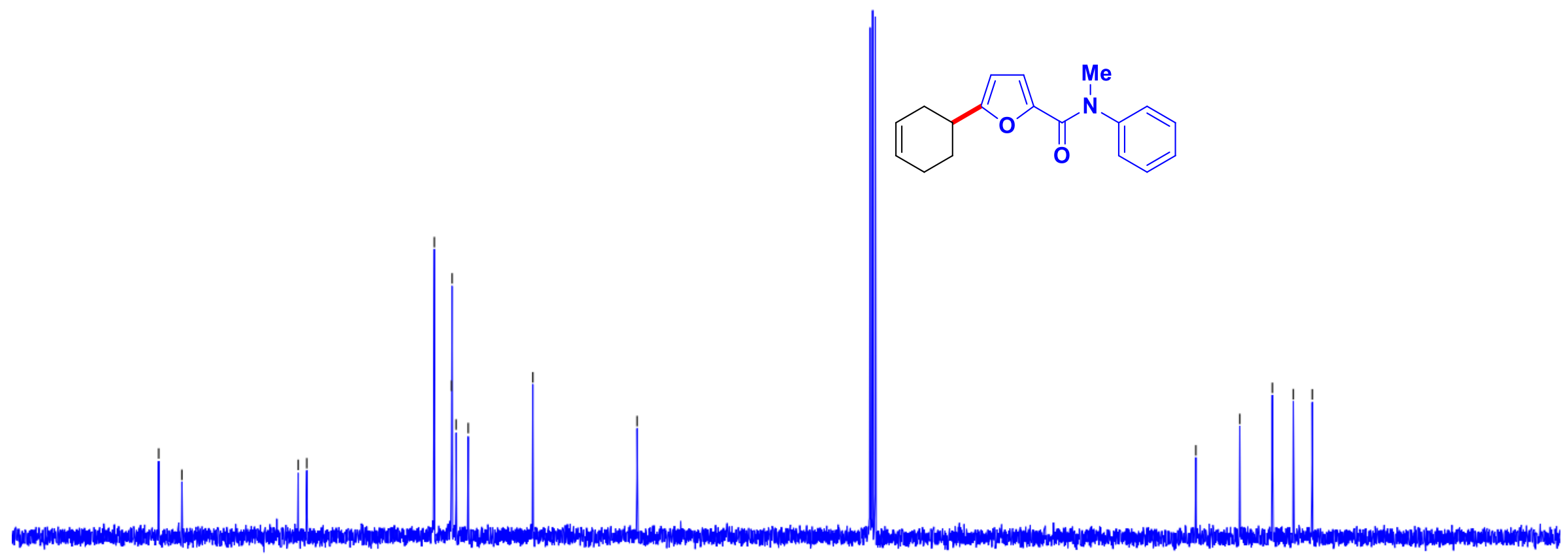

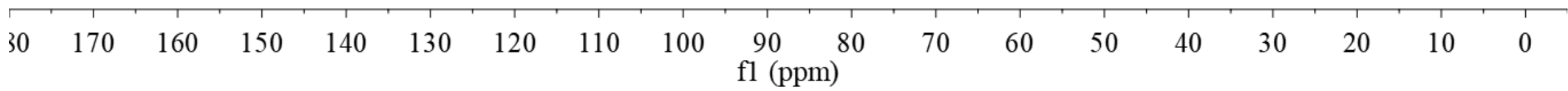


Figure S-74. ${ }^{1} \mathrm{H}$ NMR spectrum of $(\mathbf{1 z})\left(\mathrm{CDCl}_{3}, 400 \mathrm{MHz}\right)$ 5-isopropyl- $N$-methyl- $N$-phenylfuran-2-carboxamide
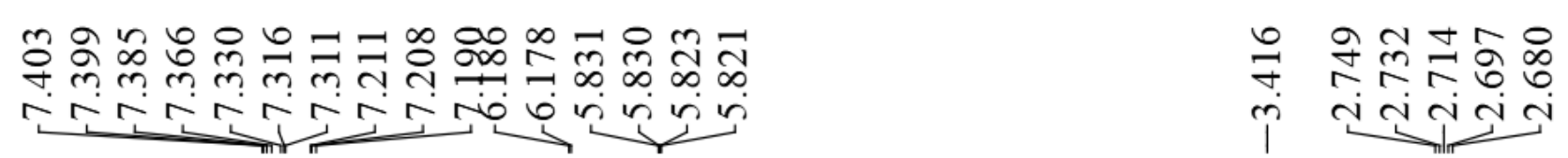

임

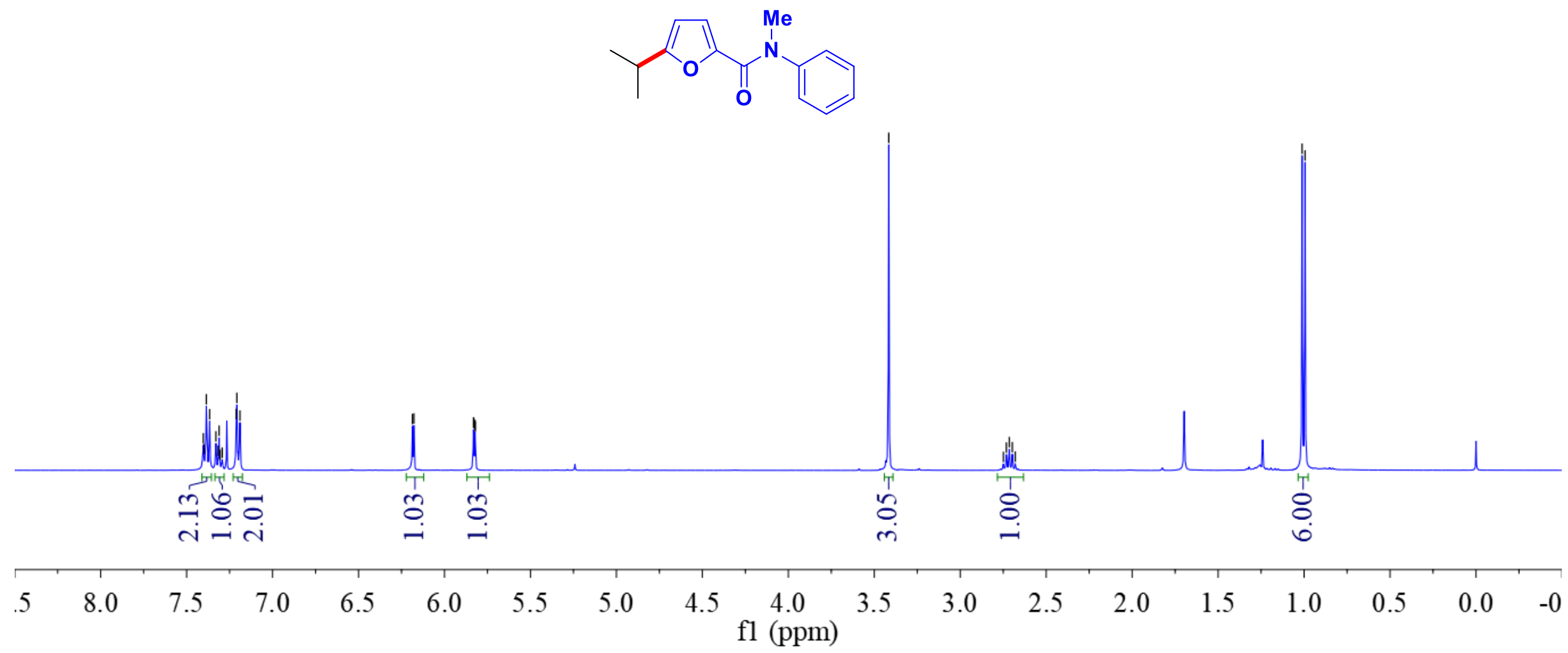


Figure S-75. ${ }^{13} \mathrm{C}\left\{{ }^{1} \mathrm{H}\right\}$ NMR spectrum of (1z) $\left(\mathrm{CDCl}_{3}, 100 \mathrm{MHz}\right)$ 5-isopropyl- $N$-methyl- $N$-phenylfuran-2-carboxamide

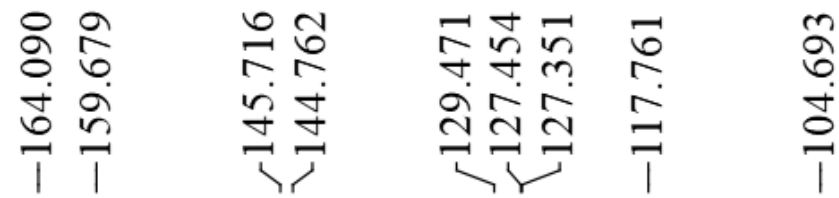

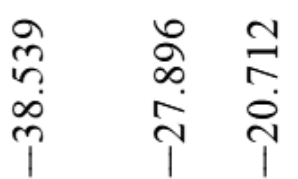

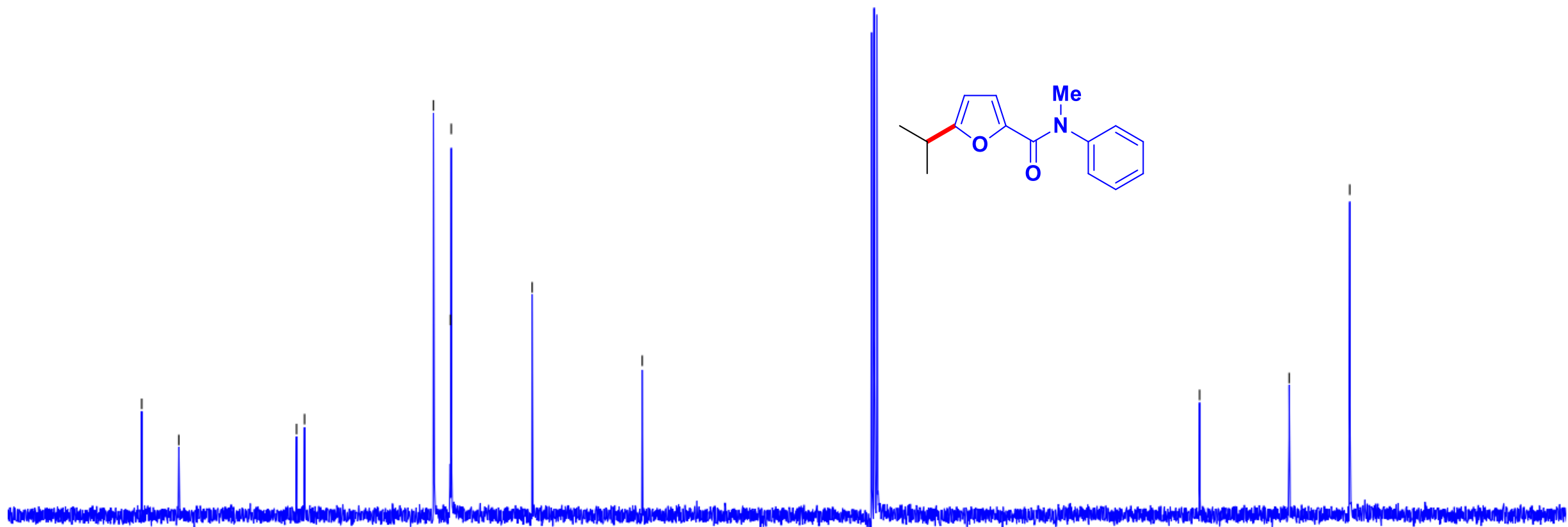

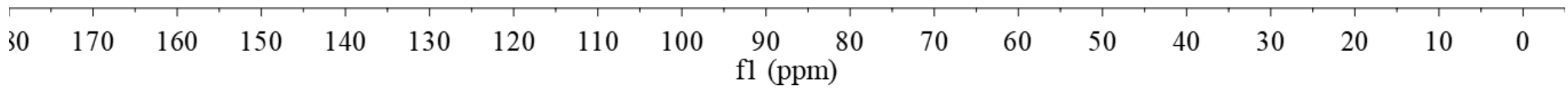


Figure S-76. ${ }^{1} \mathrm{H}$ NMR spectrum of (1aa) $\left(\mathrm{CDCl}_{3}, 400 \mathrm{MHz}\right)$ 5-(heptan-3-yl)- $N$-methyl- $N$-phenylfuran-2-carboxamide
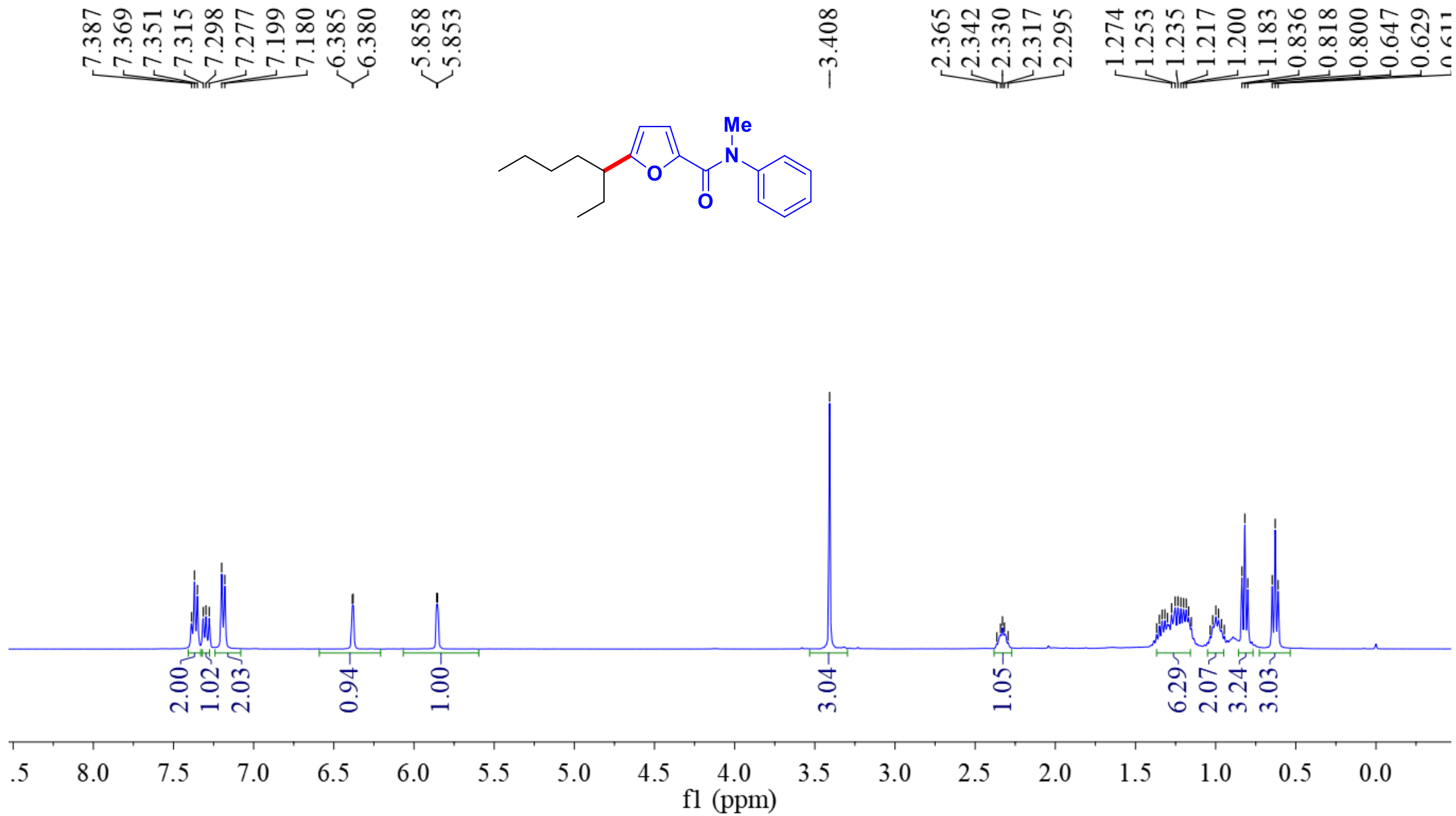
Figure S-77. ${ }^{13} \mathrm{C}\left\{{ }^{1} \mathrm{H}\right\}$ NMR spectrum of (1aa) $\left(\mathrm{CDCl}_{3}, 100 \mathrm{MHz}\right)$ 5-(heptan-3-yl)- $N$-methyl- $N$-phenylfuran-2-carboxamide

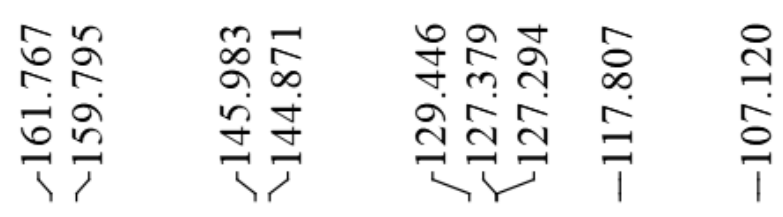

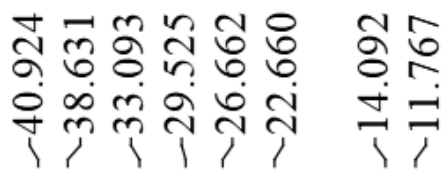<smiles>CCCCC(CC)c1ccc(C(=O)N(C)c2ccccc2)o1</smiles>

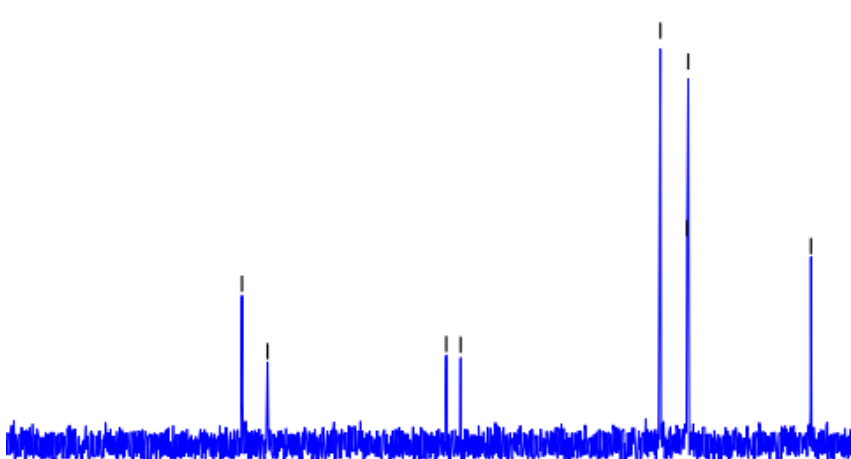

\begin{tabular}{|c|c|c|c|c|c|c|c|c|c|c|c|c|c|c|c|c|}
\hline 170 & 160 & 150 & 140 & 130 & 120 & 110 & 100 & $\begin{array}{c}90 \\
\text { fl (ppr }\end{array}$ & 80 & 70 & 60 & 50 & 40 & 30 & 20 & 10 \\
\hline
\end{tabular}


Figure S-78. ${ }^{1} \mathrm{H}$ NMR spectrum of (1ab) $\left(\mathrm{CDCl}_{3}, 400 \mathrm{MHz}\right) \boldsymbol{N}$-methyl-5-(pentan-3-yl)- $N$-phenylfuran-2-carboxamide

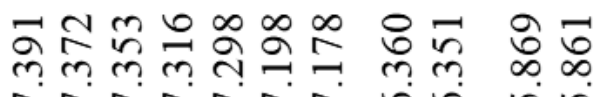

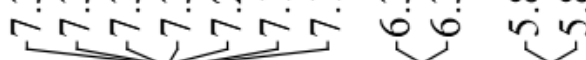
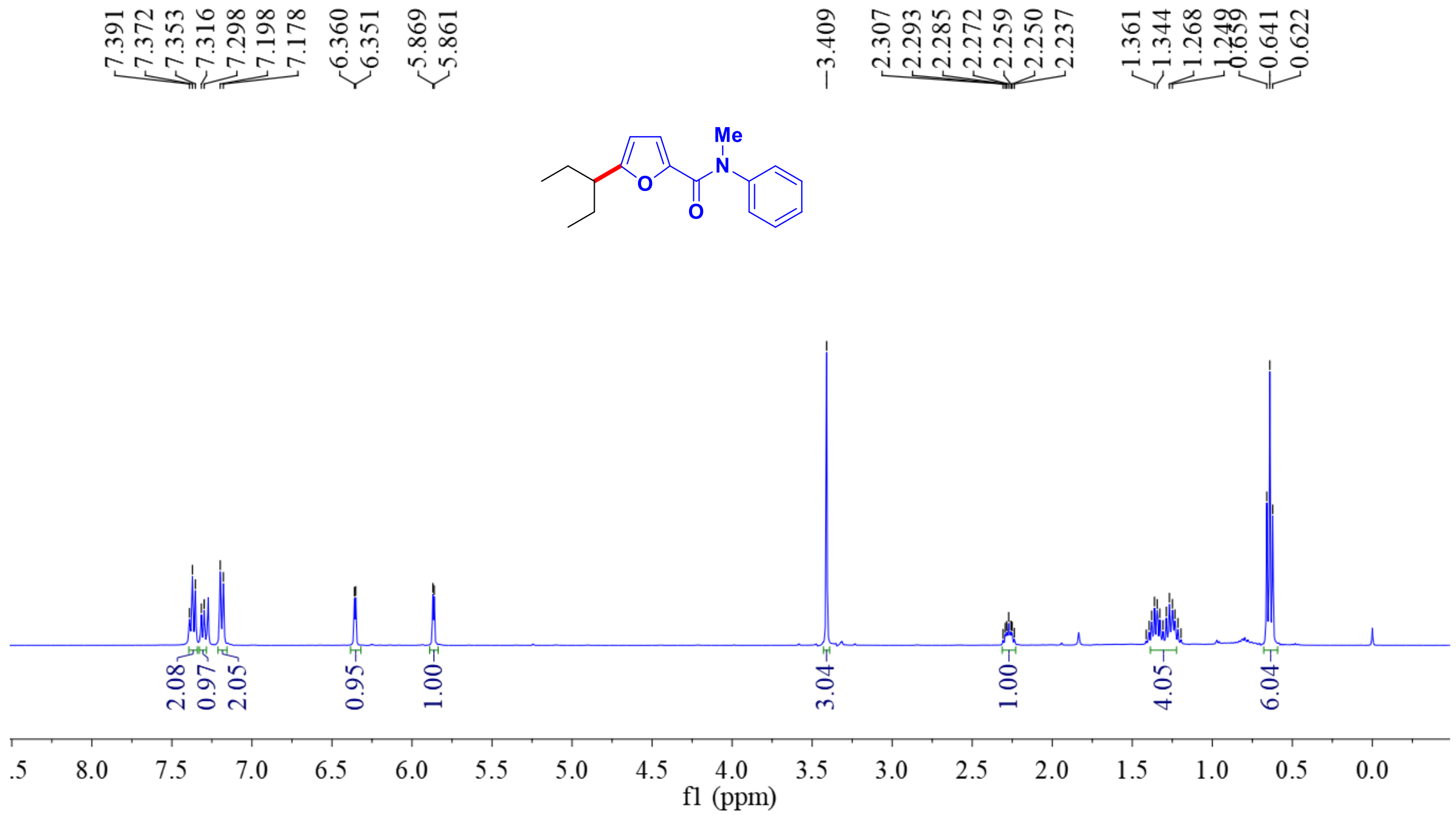
Figure S-79. ${ }^{13} \mathrm{C}\left\{{ }^{1} \mathrm{H}\right\}$ NMR spectrum of (1ab) $\left(\mathrm{CDCl}_{3}, 100 \mathrm{MHz}\right) \mathrm{N}$-methyl-5-(pentan-3-yl)- $N$-phenylfuran-2-carboxamide

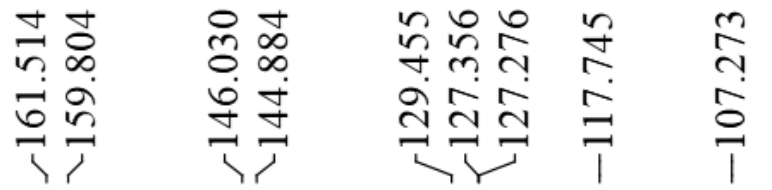

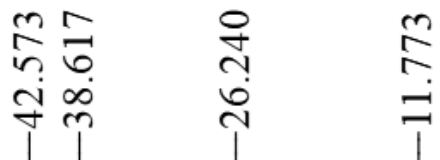
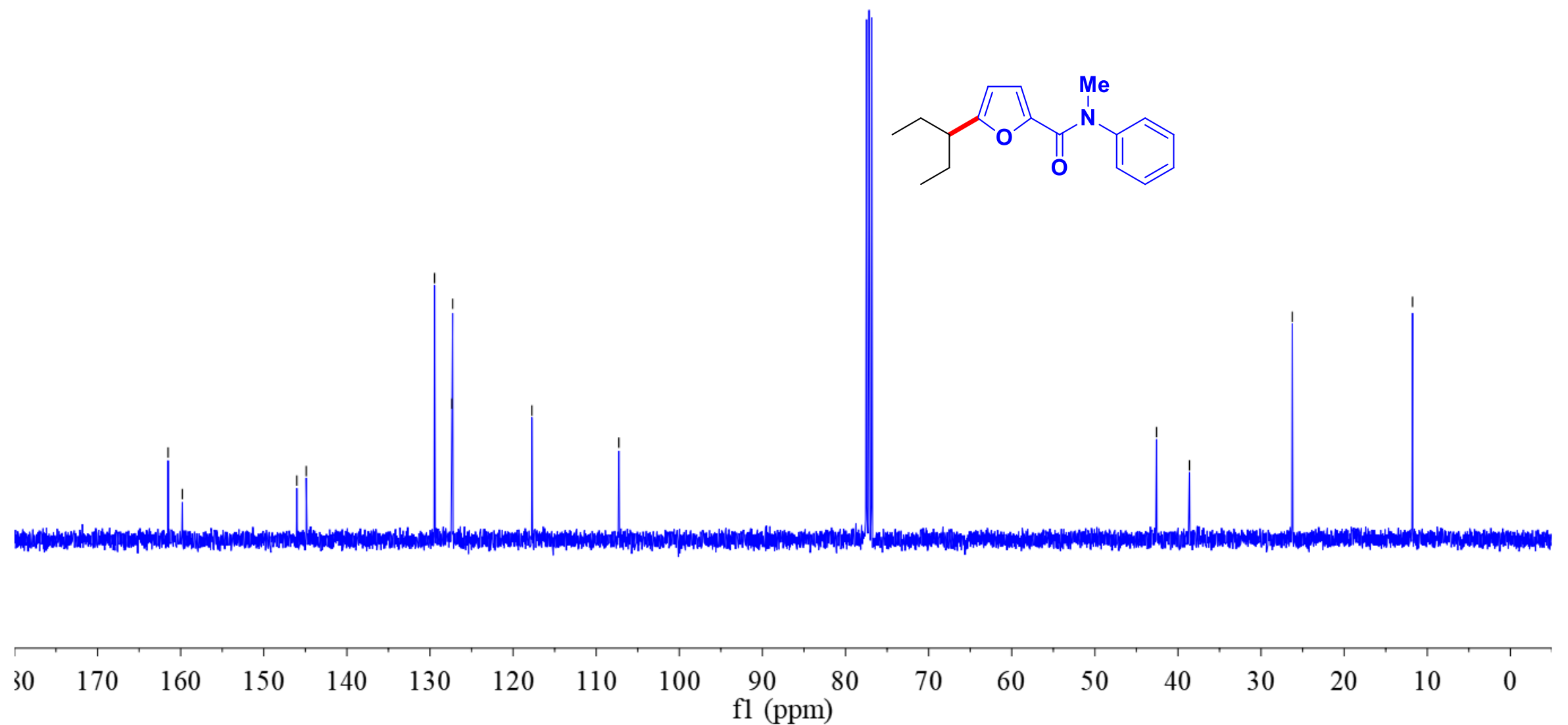
Figure S-80. ${ }^{1} \mathrm{H}$ NMR spectrum of (1ac) $\left(\mathrm{CDCl}_{3}, 400 \mathrm{MHz}\right) \mathrm{N}$-methyl-5-(pentan-2-yl)- $N$-phenylfuran-2-carboxamide ๗ลํำ
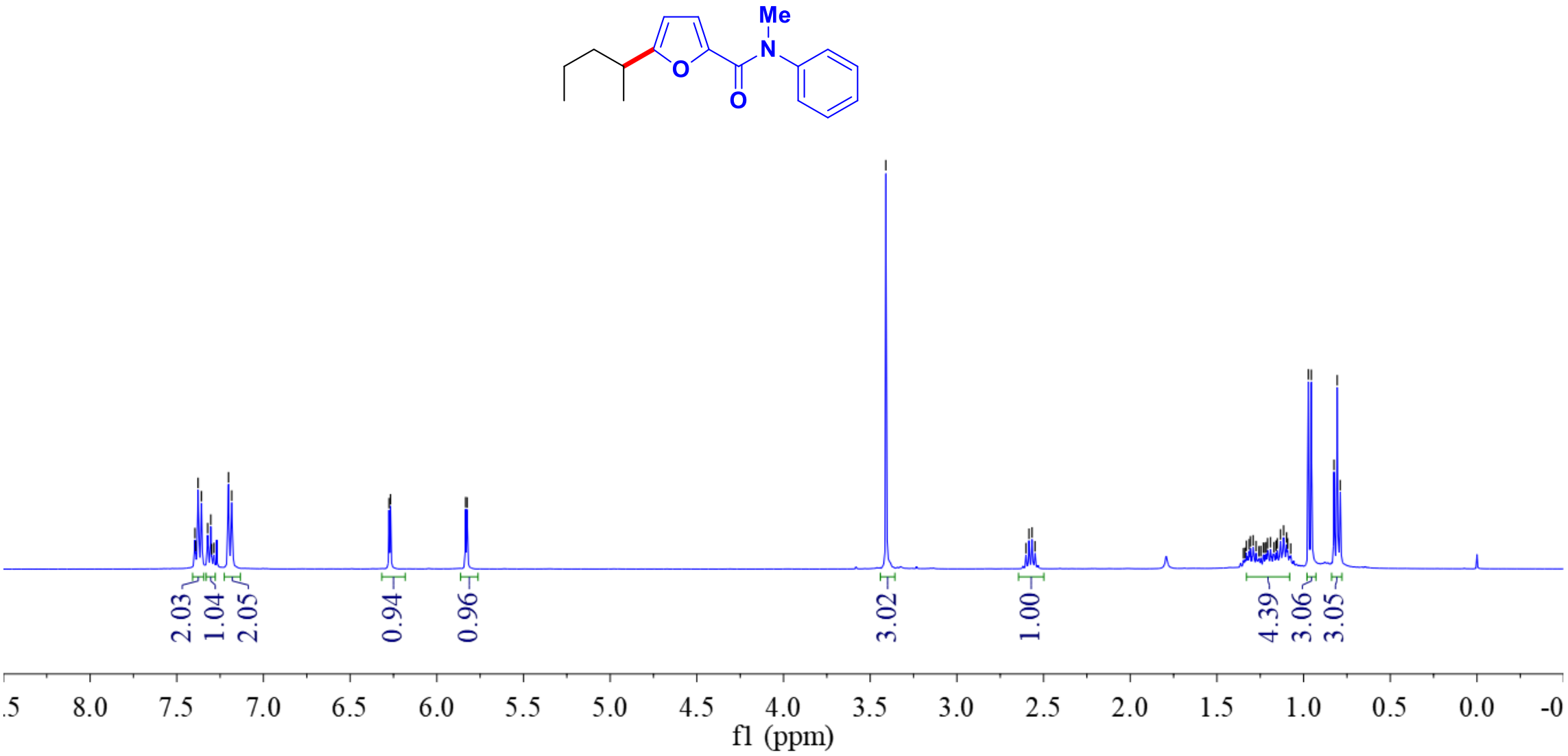
Figure S-81. ${ }^{13} \mathrm{C}\left\{{ }^{1} \mathrm{H}\right\}$ NMR spectrum of (1ac) $\left(\mathrm{CDCl}_{3}, 100 \mathrm{MHz}\right) \boldsymbol{N}$-methyl-5-(pentan-2-yl)- $N$-phenylfuran-2-carboxamide

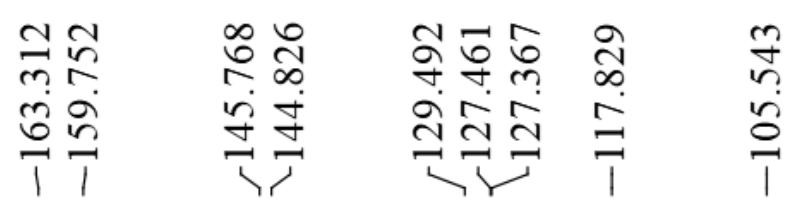

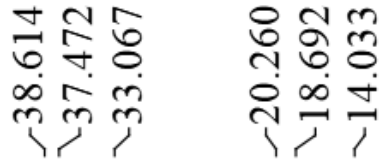

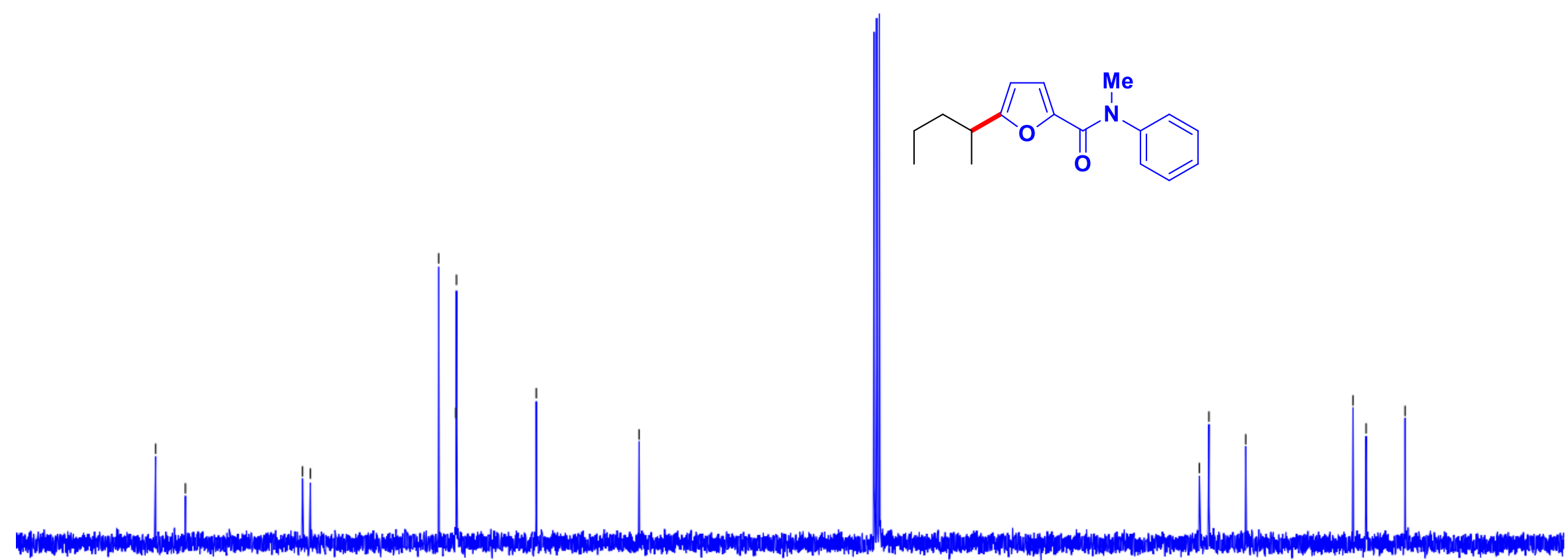

\begin{tabular}{|c|c|c|c|c|c|c|c|c|c|c|c|c|c|c|c|c|}
\hline 170 & 160 & 150 & 140 & 130 & 120 & 110 & 100 & $\begin{array}{c}90 \\
\text { fl (ppr }\end{array}$ & 80 & 70 & 60 & 50 & 40 & 30 & 20 & 10 \\
\hline
\end{tabular}


Figure S-82. ${ }^{1} \mathrm{H}$ NMR spectrum of (1ad) $\left(\mathrm{CDCl}_{3}, 400 \mathrm{MHz}\right) \mathbf{5}$-(sec-butyl)- $N$-methyl- $N$-phenylfuran-2-carboxamide
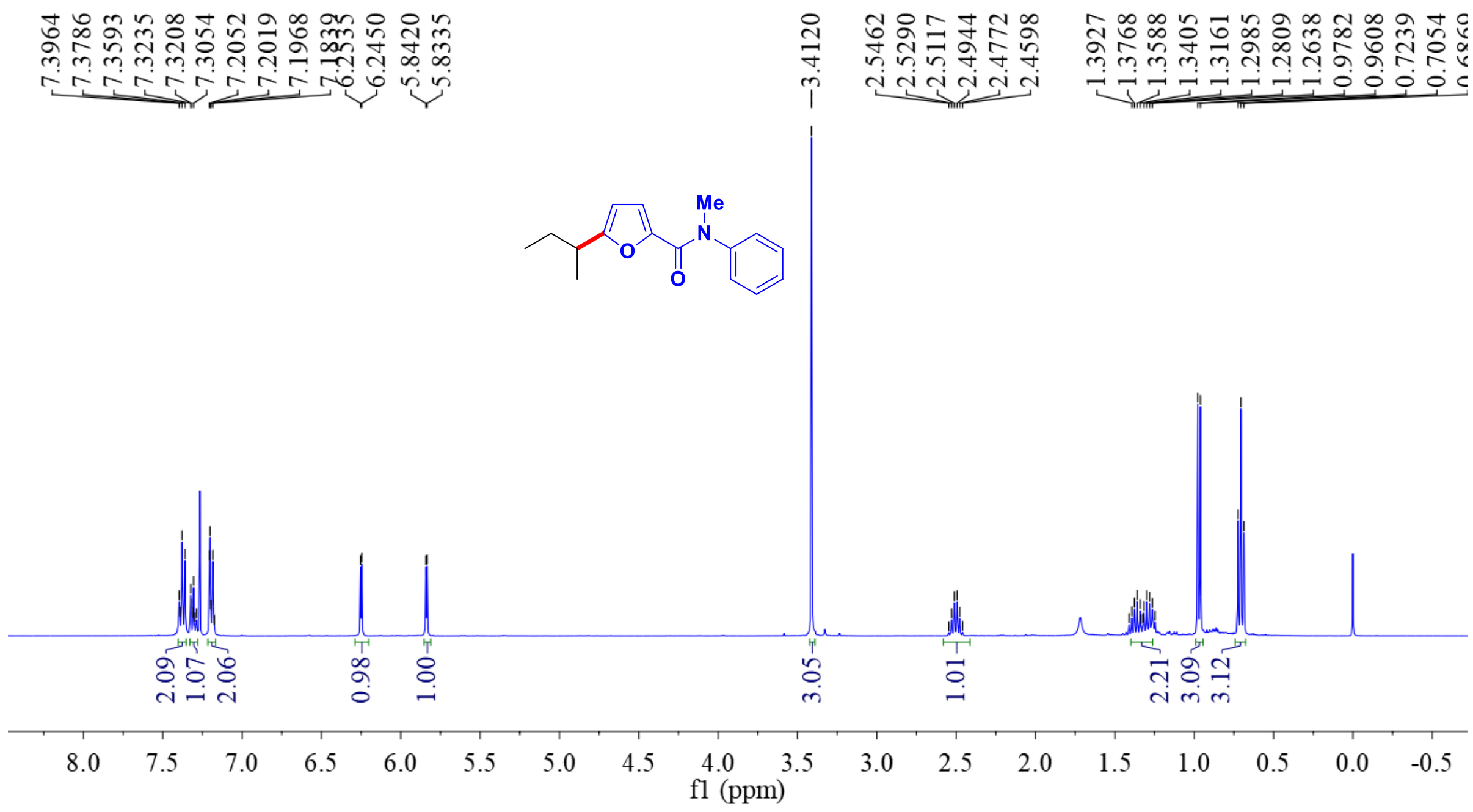
Figure S-83. ${ }^{13} \mathrm{C}\left\{{ }^{1} \mathrm{H}\right\}$ NMR spectrum of (1ad) $\left(\mathrm{CDCl}_{3}, 100 \mathrm{MHz}\right)$ 5-(sec-butyl)- $N$-methyl- $N$-phenylfuran-2-carboxamide

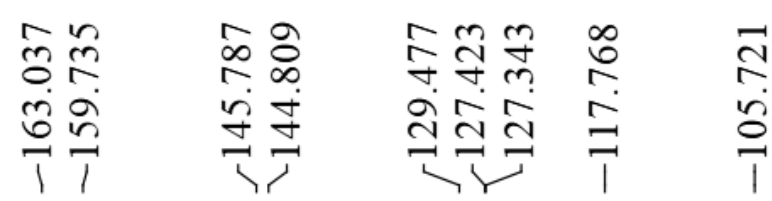

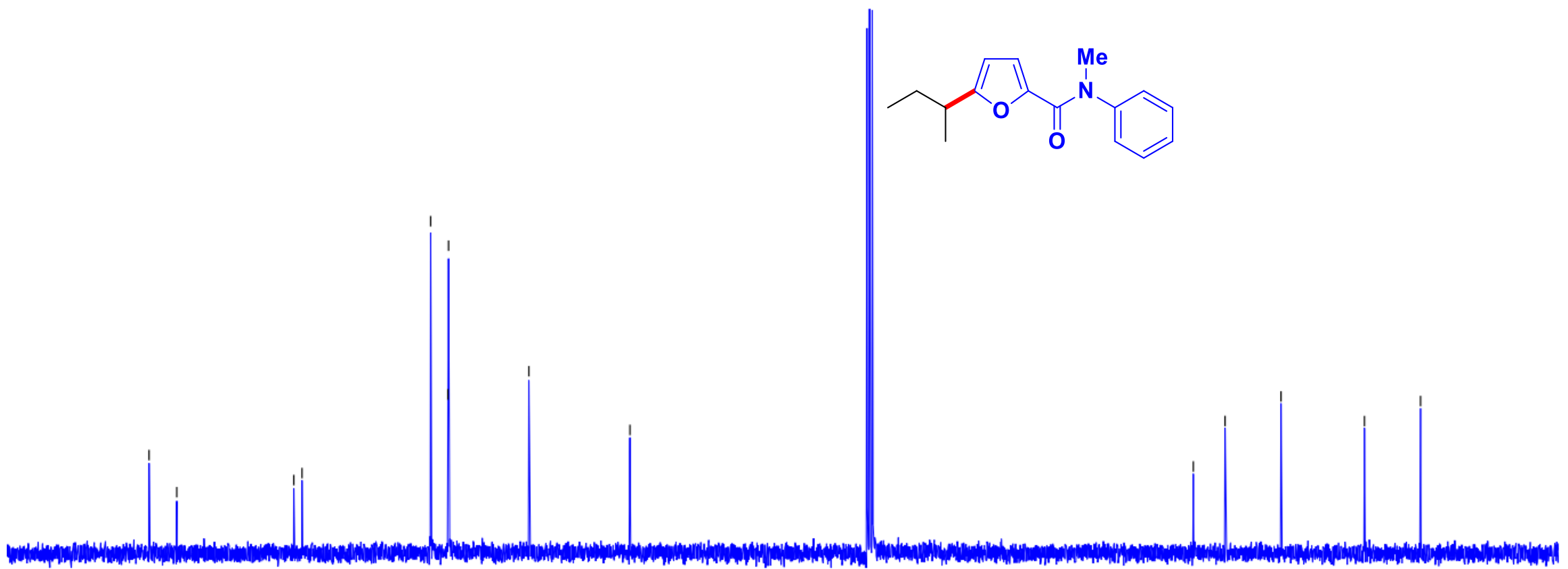

$30 \quad 170$

160

150

140

130

120

110

100

$90 \quad 80$

fl (ppm)

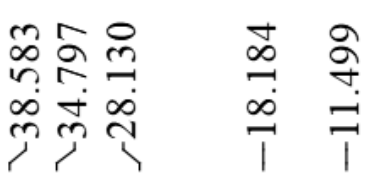


Figure S-84. ${ }^{1} \mathrm{H}$ NMR spectrum of (1ae) $\left(\mathrm{CDCl}_{3}, 400 \mathrm{MHz}\right) \mathrm{N}$-methyl- $N$-phenyl-5-propylfuran-2-carboxamide
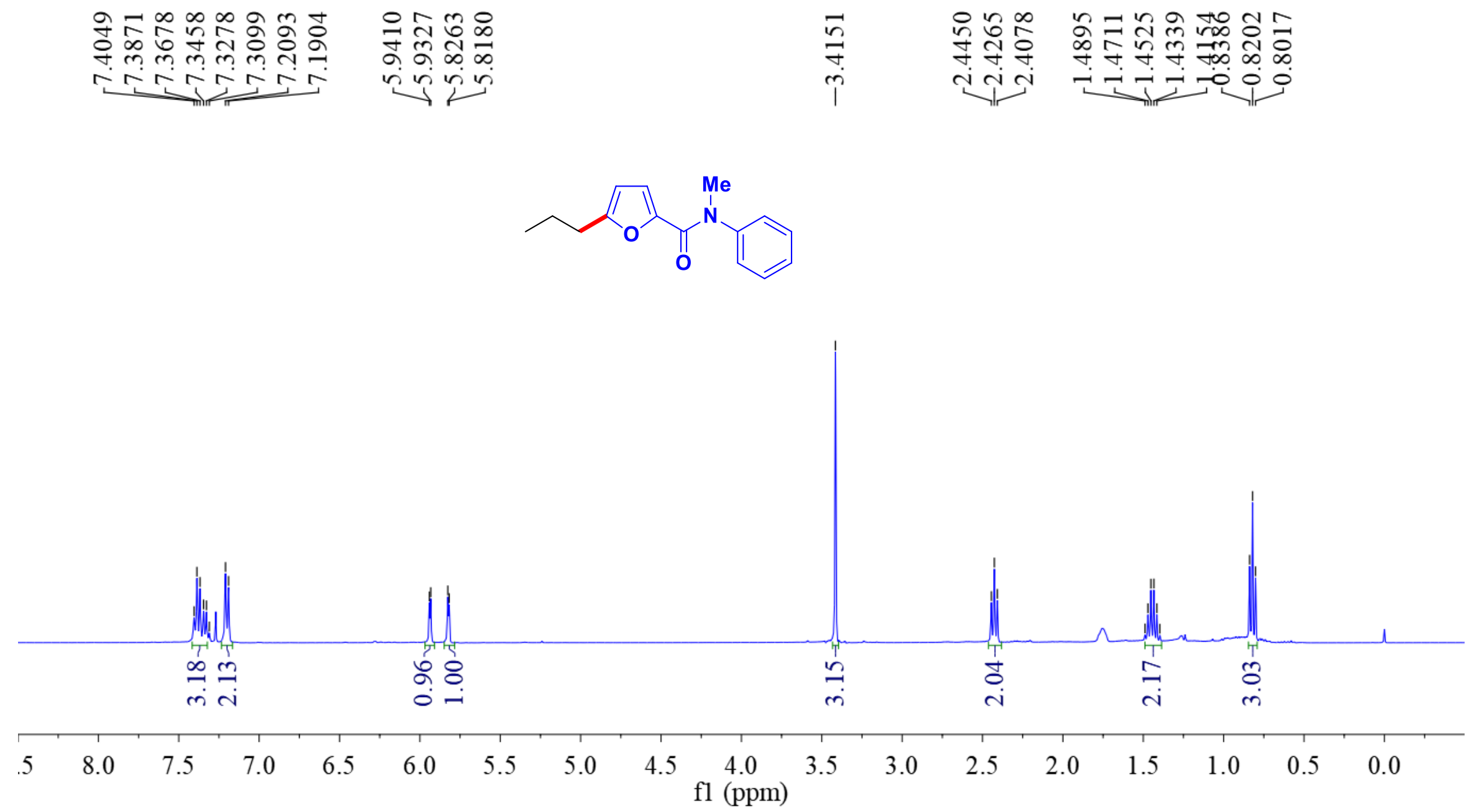
Figure S-85. ${ }^{13} \mathrm{C}\left\{{ }^{1} \mathrm{H}\right\}$ NMR spectrum of (1ae) $\left(\mathrm{CDCl}_{3}, 100 \mathrm{MHz}\right) \mathrm{N}$-methyl- $N$-phenyl-5-propylfuran-2-carboxamide

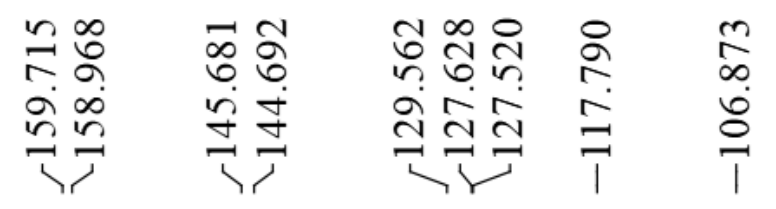

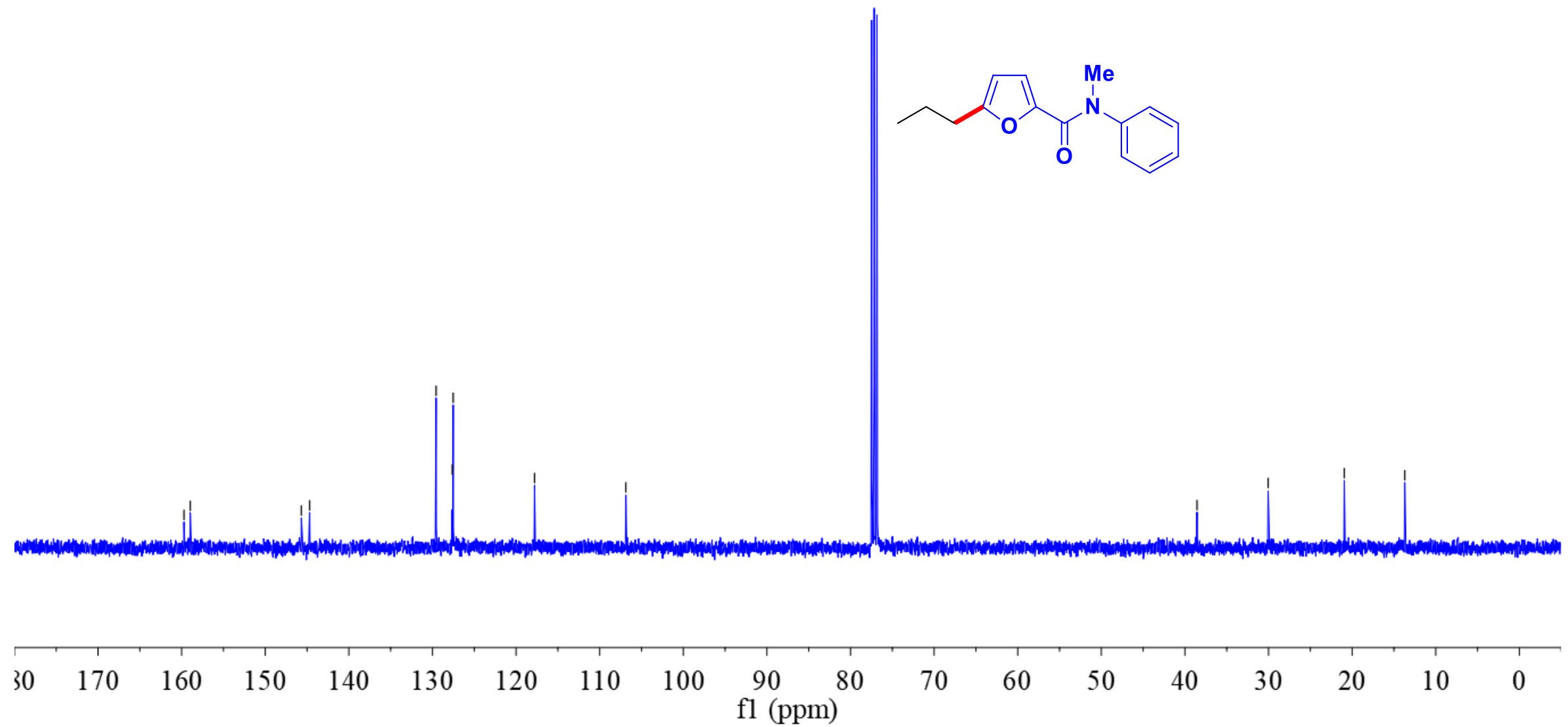


Figure S-86. ${ }^{1} \mathrm{H}$ NMR spectrum of (1af) $\left(\mathrm{CDCl}_{3}, 400 \mathrm{MHz}\right)$ (1af) 5-isobutyl- $N$-methyl- $N$-phenylfuran-2-carboxamide

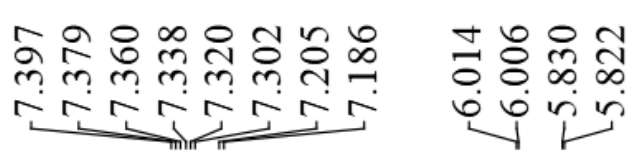
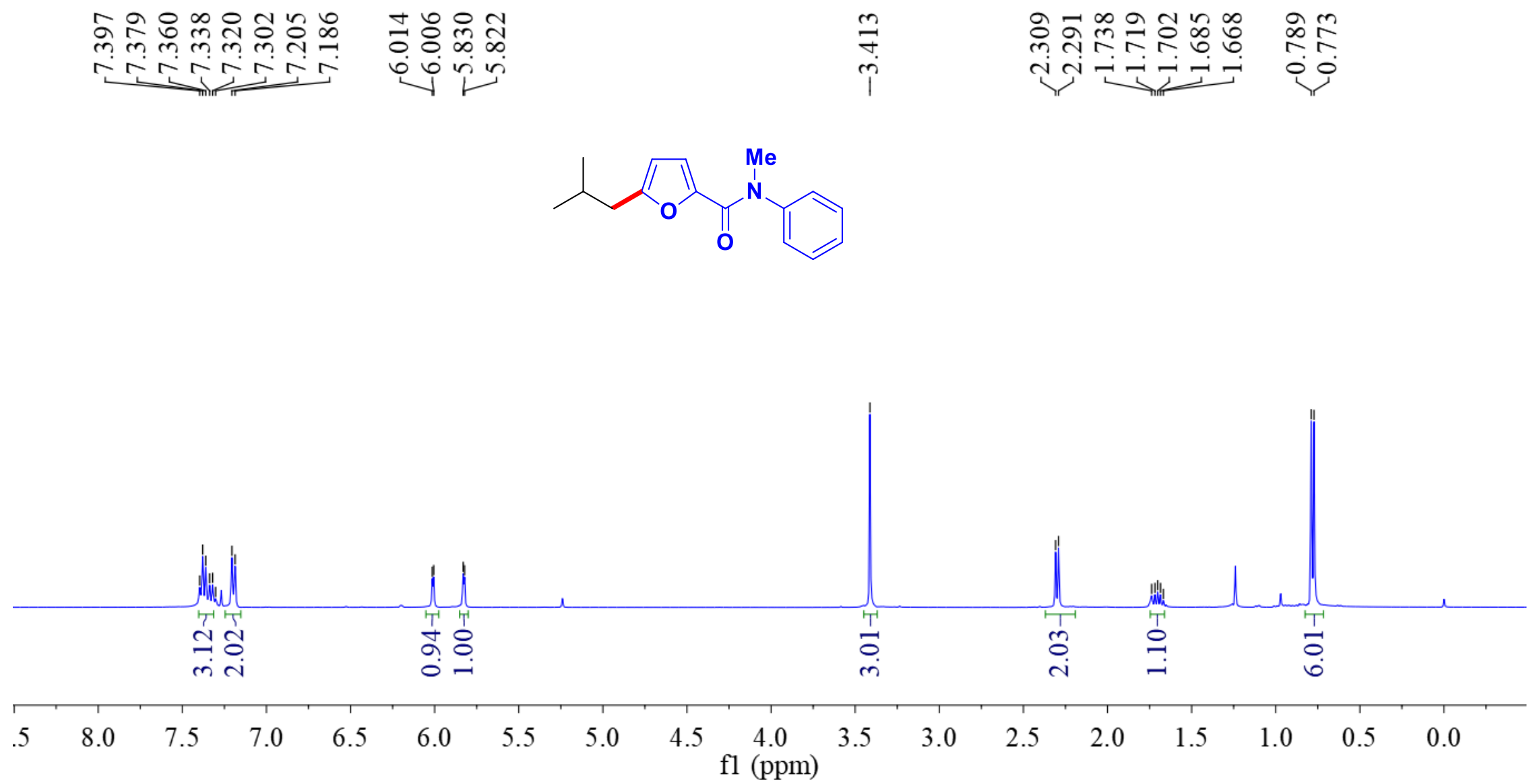
Figure S-87. ${ }^{13} \mathrm{C}\left\{{ }^{1} \mathrm{H}\right\}$ NMR spectrum of (1af) $\left(\mathrm{CDCl}_{3}, 100 \mathrm{MHz}\right)$ 5-isobutyl- $N$-methyl- $N$-phenylfuran-2-carboxamide

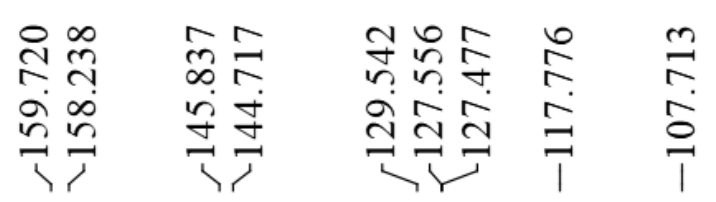

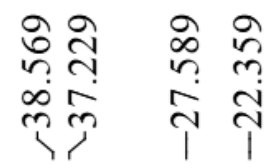
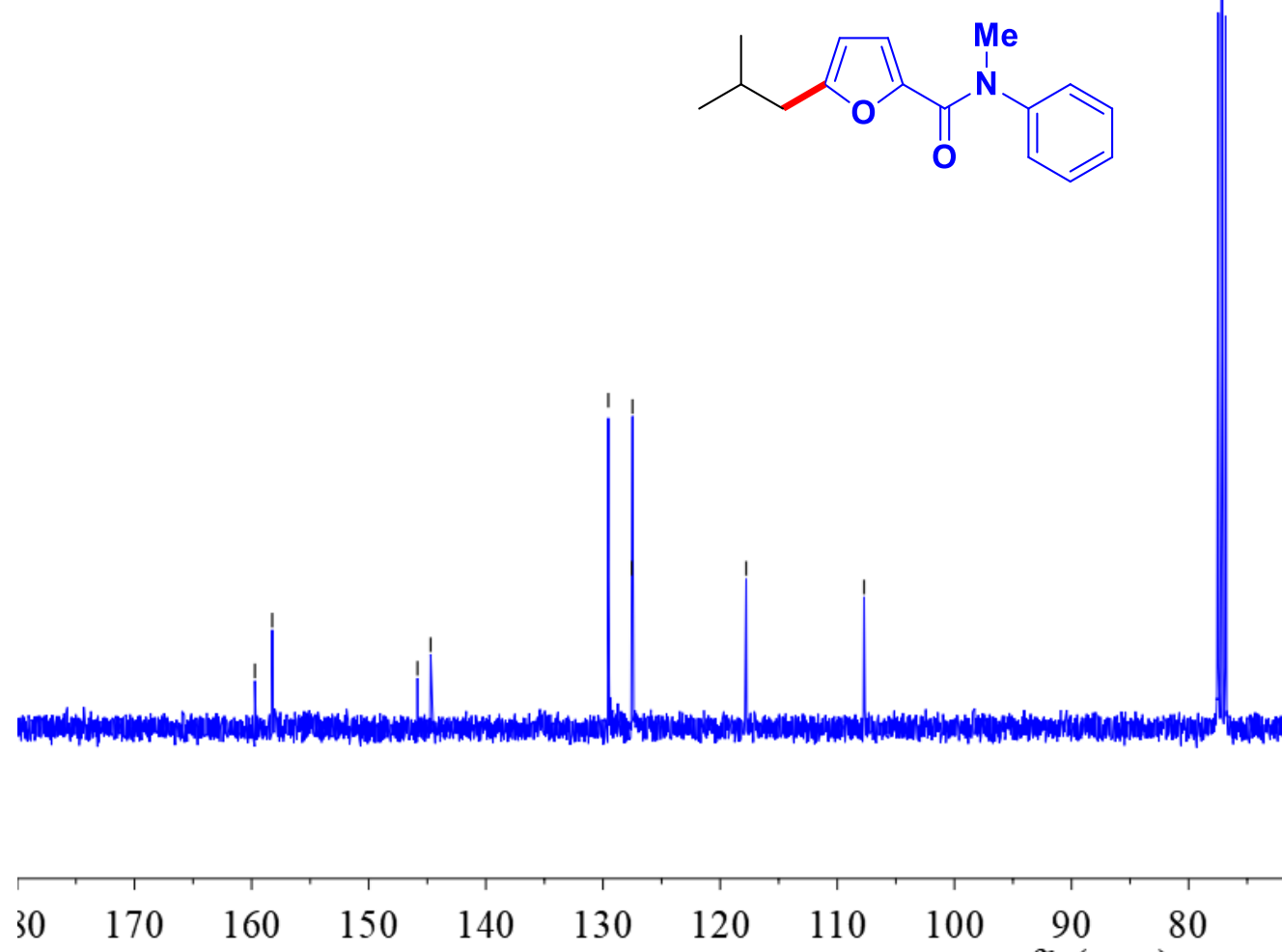

fl (ppm) 
Figure S-88. ${ }^{1} \mathrm{H}$ NMR spectrum of (1ag) $\left(\mathrm{CDCl}_{3}, 400 \mathrm{MHz}\right)$ 5-butyl- $N$-methyl- $N$-phenylfuran-2-carboxamide

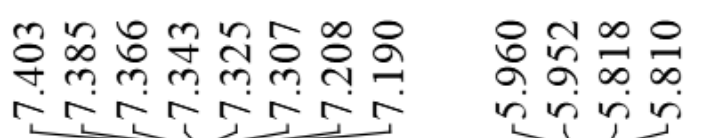
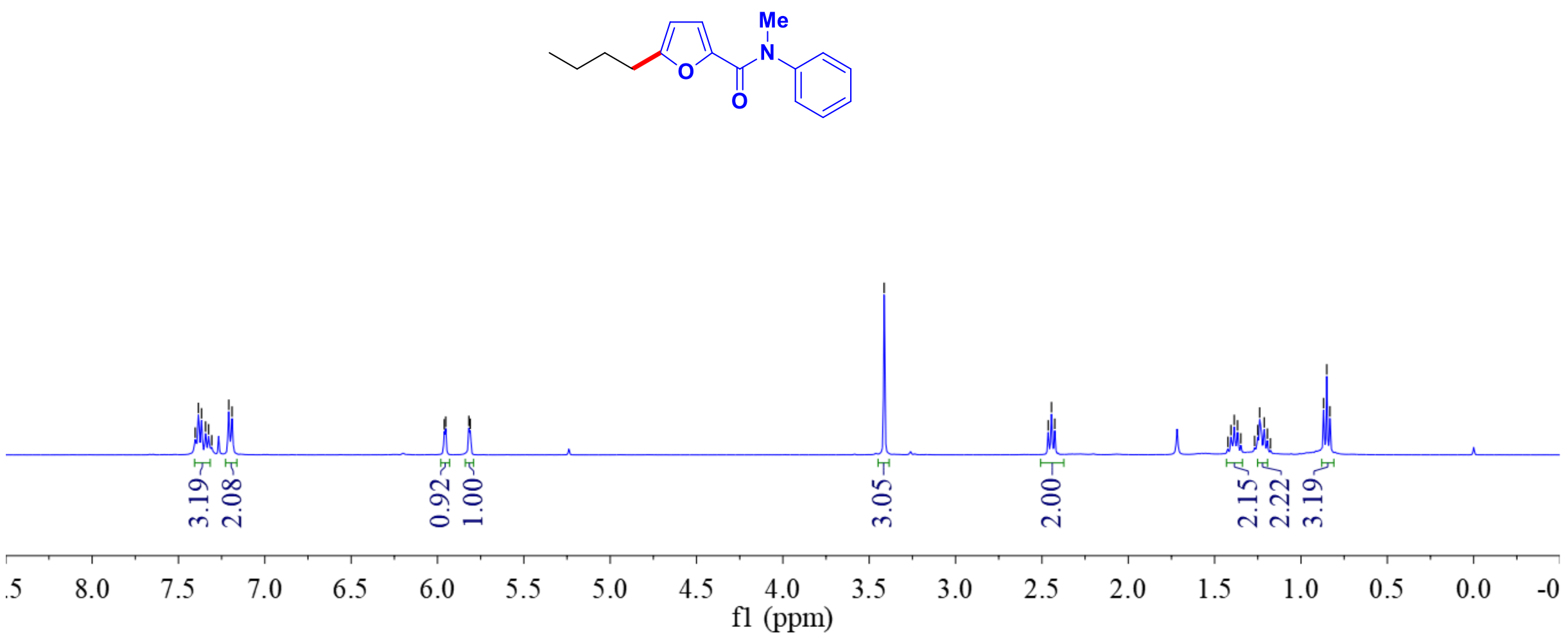
Figure S-89. ${ }^{13} \mathrm{C}\left\{{ }^{1} \mathrm{H}\right\}$ NMR spectrum of (1ag) $\left(\mathrm{CDCl}_{3}, 100 \mathrm{MHz}\right)$ 5-butyl- $N$-methyl- $N$-phenylfuran-2-carboxamide

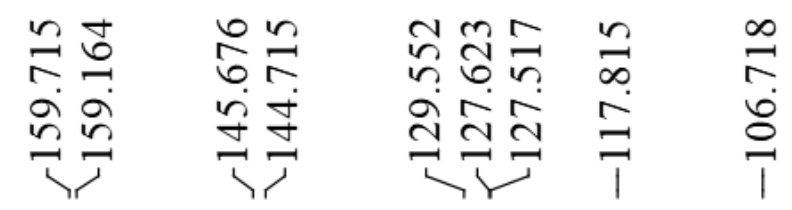

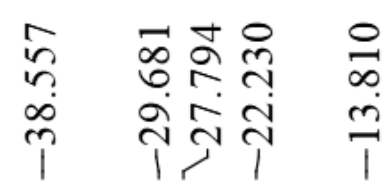

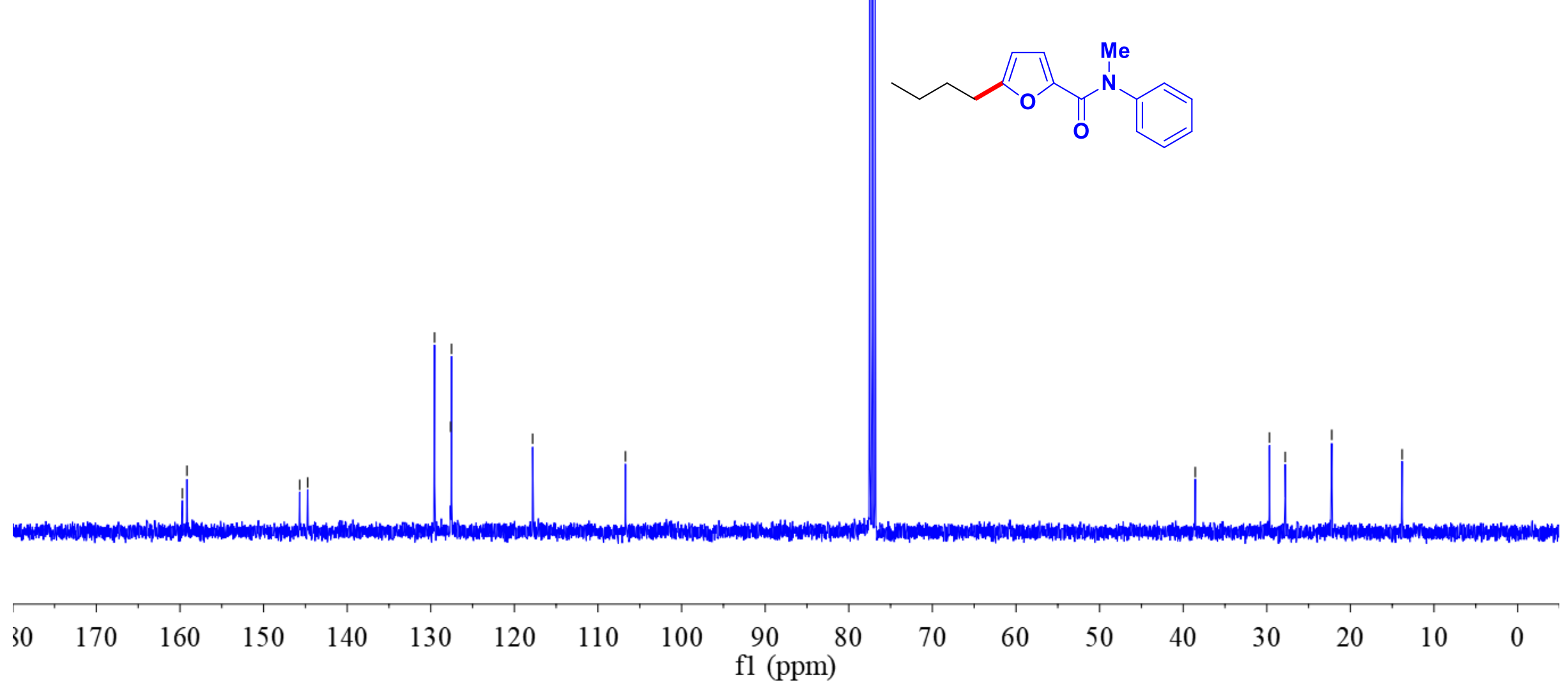




\section{Control experiments}

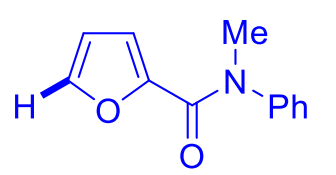

$2 a$

1 equiv

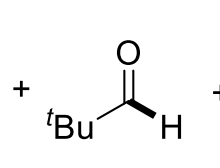

$3 a$

5 equiv 3 equiv $\underset{\mathrm{Ph}}{\stackrel{\mathrm{FeCl}_{2}(0.05 \text { equiv })}{\mathrm{DTBP}(3 \text { equiv })}}$

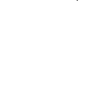

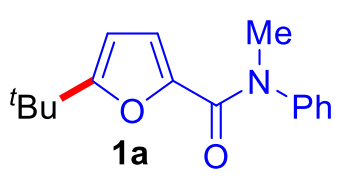

$21 \%$

$2 a$

recovered, $71 \%$
${ }^{t} \mathrm{Bu}$ ${ }_{4 a} \mathrm{Ph}$ Detected by GC-MS

Figure S-90. the product of alkyl radical trapping (4a)
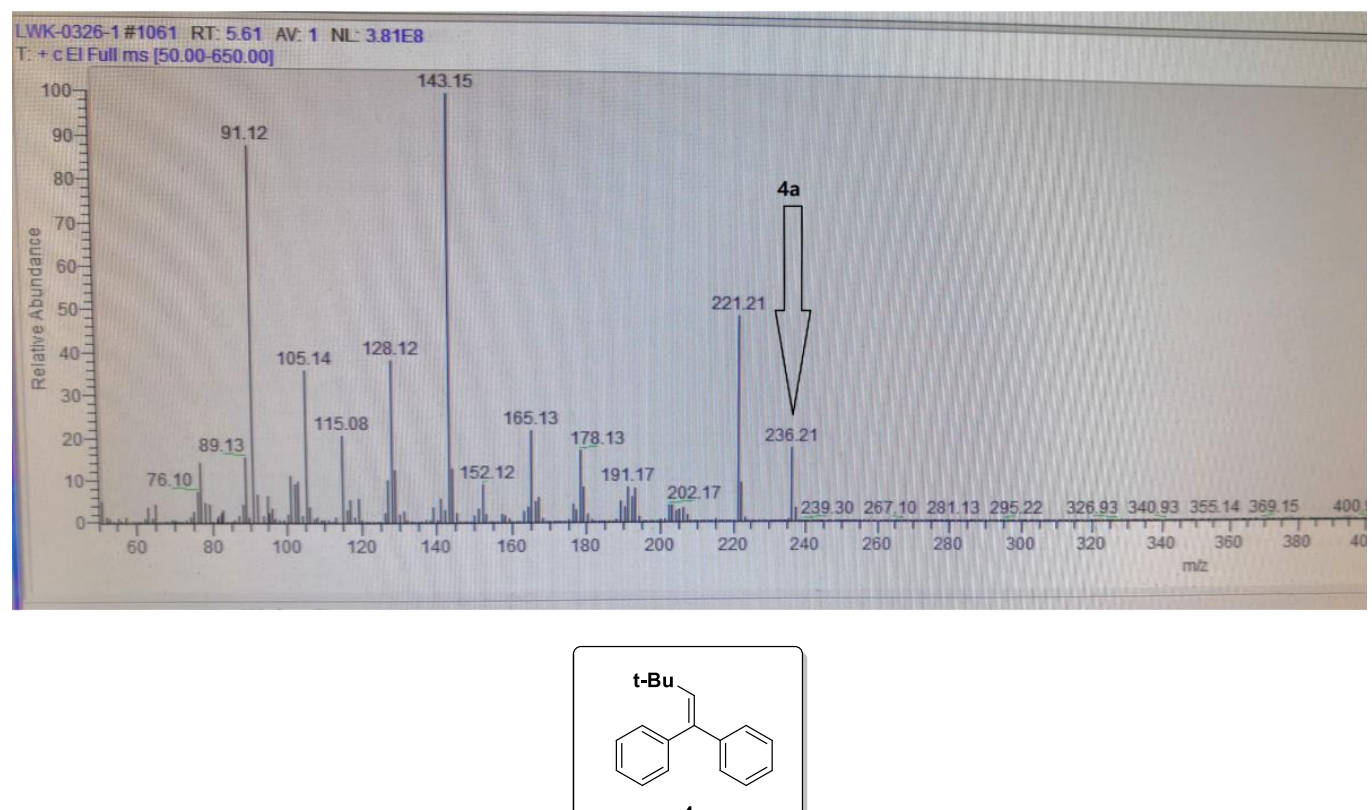

4a 